Project No. 09-818

\title{
Simulations of Failure via Three- Dimensional Cracking in Fuel Cladding for Advanced Nuclear Fuels
}

\section{Fuel Cycle RD\&D}

Dr. Hongbing Lu University of Texas, Dallas

In collaboration with:

Bardenhagen Consulting Oklahoma State University University of Tennessee at Knoxville

Frank Goldner, Federal POC Cetin Unal, Technical POC

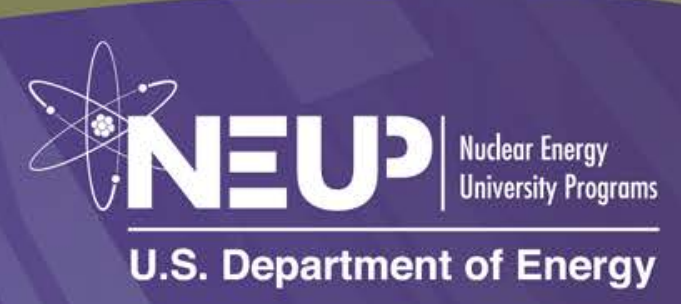


Simulations of Failure via Three-Dimensional Cracking in Fuel Cladding for Advanced Nuclear Fuels

Project Number: 09-416

PI: Hongbing Lu

(co-PIs) Raman P. Singh, Scott Bardenhagen

Ranga Komanduri (Late)

Submitted on November 30, 2013 


\section{Contents}

1 Introduction 5

2 Material Point Method (MPM) 6

2.1 Higher Order Shape Functions in 2D GIMP $\ldots \ldots \ldots \ldots \ldots$

2.2 Failure Pattern Analysis of Bulk Failure in Cladding . . . . . . . . . . . . . . . . 29

2.3 Discretization Errors in the Material Point Method . . . . . . . . . . . . . . . 44

2.4 Implementation of Convected Particle Domain Interpolation (CPDI) for Error Reduction 49

3 Simulation of Fracture in Irradiated Iron using Molecular Dynamics 52

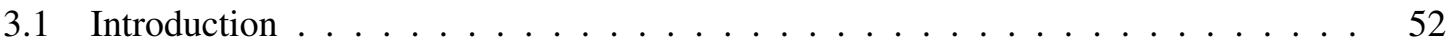

3.2 Computational Methodology . . . . . . . . . . . . . . . . 53

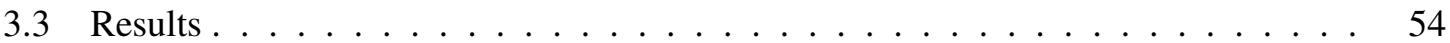

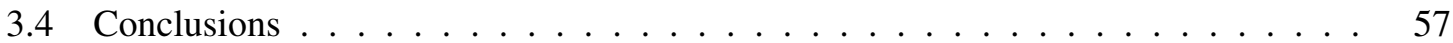




\section{Publications and Presentations}

The following are the publications and conference presentations made along the course of this project. Additional journal papers will be submitted.

1. S. Bardenhagen, J.A. Nairn, and H. Lu, "Simulation of dynamic fracture with the Material Point Method using a mixed Jintegral and cohesive law approach”, International Journal of Fracture, 170, 49-66 (2011).

2. J. Ma, J.C. Hanan, R. Komanduri, and H. Lu, "Simulation of the deformation mechanisms of bulk metallic glass (BMG) foam using the Material Point Method", Computer Modeling in Engineering and Sciences, 86, 349-384 (2012).

3. Z. Hu, H. Luo, Y. Du, and H. Lu, "Fluorescent stereo microscopy for 3D surface profilometry and deformation mapping”, Optics Express, 21, 11808-11818 (2013).

4. H. Luo, W.L. Cooper, H. Lu, "Effects of particle size and moisture on the compressive behavior of dense Eglin sand under confinement at high strain rates", International Journal of Impact Engineering, 65, 40-55 (2014).

5. Z. Hu, Y. Du, H. Luo, B. Zhong, and H. Lu, "Internal deformation measurement of Mason sand under confined compression using incremental Digital Volume Correlation, (Journal paper, submitted).

6. "Characterization of the grain-Level mechanical behavior of Eglin sand by nanoindentation", F. Wang, B. Fu, H. Luo, S. Staggs, R.A. Mirshams, W.L. Cooper, S.Y. Park, M.J. Kim, C. Hartley, H. Lu (Journal paper, submitted).

7. Z. Hu, H. Luo, S.G. Bardenhagen, C.R. Siviour, R.W. Armstrong, and H. Lu, "Internal deformation measurement of polymer bonded sugar in compression by Digital Volume Correlation of in-situ Tomography", (Journal paper, submitted).

8. H. Lu, S. Bardenhagen, and J.A. Nairn, "Dynamic fracture simulations in MPM using a J-Integral approach", World Conference on Computational Mechanics, Jul. 19-23 (2010). 
9. S. Bardenhagen, J.A. Nairn, and H. Lu, "Dynamic fracture simulations in MPM using a J-Integral approach", $6^{\text {th }}$ Material Point Method Workshop, Aug. 8-9 (2010).

10. C. Mollamahmutoglu and H. Lu, "Evaluation of higher order shape function in 2D GIMP simulations", $11^{\text {th }}$ US National Congress on Computational Mechanics, Jul. 25-28 (2011).

11. V. Subramanian, R.P. Singh, and H. Lu, "Simulation of fracture in irradiated iron using Molecular Dynamics", Annual Conference of the American Nuclear Society, Nov. 10-14 (2013). 


\section{Introduction}

The three major fuels comprising irradiation used in the U.S. are mixed oxide, metal, and mixed carbide rods. Over several decades of efforts, factors affecting safety in operation have been identified, and addressed to a certain degree. As a result, all thee fuel types do not appear to pose life threatening dangers. The enhancement in the performance of fuel cladding and duct alloys has become a major area of improvement to increase the fuel burnup. Under the GNEP (Global Nuclear Energy Partnership) the DoE national labs have been directing towards working on the advanced burner reactor in which spent fuel is transmutated into actinides, having much shorter irradiation half-life. In the fast fuel used in advanced burner reactors, simulations of the nuclear fuels are critical in understanding the burnup and thus the fuel efficiency. In these simulations the important issue is on the determination of the failure of the fuel cladding through three-dimensional cracking. Of lesser importance is the cracking of the nuclear fuel itself, as the cracks are considered not to affect to a significant extent the fuel to perform its functions.

Fuel cladding is made of materials, such as stainless steels and ferritic steels with added alloying elements to increase its stability under irradiation and thus durability. Fuel cladding is subject to water, chemicals, fission gas, pressure, high temperature and irradiation in service. Understanding its performance in the fast fuel reactors is essential. DoE labs have been working on the mechanical behavior of fuel cladding, mostly without specifically considering the failure via three-dimensional cracking. Limited work is available in the literature on fracture simulations of cladding, extensive combined simulations and experimentations are not available in this area, such as crack surface profile, friction at the crack surfaces necessary for simulations. This project addresses the failure of the fuel cladding via three-dimensional cracking. Simulations of the failure of the fuel cladding are a complex problem, due to issues such as: (1) grain boundary or texture evolution due to diffusion of materials under stress and temperature; (2) non-uniform alloy reformation in regions due to diffusion, resulting in regions with low melt point, causing thinning of the cladding wall; (3) growth or swelling of cladding in axial and circumferential directions due to irradiation creep, resulting 
in embrittlement in material behavior; (4) anisotropy in properties in the axial and circumferential directions; (5) coupled multiphysics phenomena involving thermal, mechanical, irradiation, fluid (liquefied fuel/cladding, fission gas); (6) large scale computations involved. In this project, we developed a simulation code for the failure of the fuel cladding, and validated it from experiments. In this report, we present details of the computational and experimental methods and their results in analysis of 3-D cracking of fuel cladding materials.

\section{Material Point Method (MPM)}

Material point method (MPM) is introduced by Sulsky et.al. It is developed from the FLIP algorithm of Los Alamos Research Group. In MPM each sub-domain is represented as a material point with a concentrated mass which is equal to the mass of the sub-domain. The material point also carries all the solution variables such as position, velocity, deformation gradient, stress etc. MPM algorithm can be roughly divided into two phases. The first phase is the interpolation phase where all the solution variables (mass, momentum, stresses) on the material points are interpolated to a background mesh. This mesh (usually a structured grid with quad elements) is solely for computational procedures and does not related with the actual material domain in any physical way. The equations of motion is solved and updated on grid nodes. The second phase is the convective phase where the grid solutions are mapped back to the particles so that new particle strains, stresses, velocities and positions are calculated. The grid will also deform with the material according to the calculated deformation field but since it is solely used for calculations a new undeformed grid is constructed for the next time step. Actually in practice grid deformation is never calculated and the new undeformed grid is taken as the initial grid for all time steps for the sake of convenience. MPM found great application in the simulation of large deformation and large strain problems thanks to its inherent mixed Eulerian-Lagrangian nature. Nonslip contact of material is also an inherent property coming with the MPM formulation without any additional effort. 


\subsection{Higher Order Shape Functions in 2D GIMP}

Mesh refinement is a vital aspect of the modern numerical methods. In order to resolve large strain gradients and stress concentrations in numerical simulations a refined mesh should be employed for regions of interest. Dynamic fracture propagation is among the problems with these specifications. Mesh refinement studies can be roughly classified in two sections: h-refinement and p-refinement. Making the mesh finer over the parts of the body is termed h-refinement. Making the element order higher is termed p-refinement. In MPM only h-refinement is considered up to date. These studies considered unstructured mesh h-refinement or structured mesh h-refinement. Although it is working properly unstructured mesh refinement is not suggested because of the readily advantages of structured meshes for numerical implantation and computational efficiency.

Ma et.al (2006) (paper attached) used SAMRAI infrastructure for structured mesh h-refinement in $2 \mathrm{D}$. They introduced a hierarchical order of refinement with rectangular patches in the GIMP framework. For this they have modified the regular 2D quad based GIMP functions for the boundaries between patches. Each rectangular patch is included in a coarser rectangular patch with an integer ratio of h-refinement (element size ratio between two adjacent patches in the hierarchical order is an integer). Thus each patch corresponds to a level of refinement. While level 0 is the coarsest and corresponds to the entire grid, level $\mathrm{n}$ ( $\mathrm{n}$ is the highest level prescribed by the user) is the finest corresponding to the smallest patch which is placed at the center of region of interest (with anticipated high strain gradient and stress concentration). Although it is quite successful the method works for only strictly convex patches (the patches should be always rectangular without any inward corner). If any patch with an inward corner is introduced proposed modified GIMP functions do not satisfy partition of unity hence yield unphysical results. Introduction of inward corners require 6-node transition cells from fine to coarse transition and the modified functions fail here. A set of serendipity functions which exactly satisfies partition of unity might be used for these transition locations. In this study instead of the serendipity functions we started to work with regular 9-node Lagrangian elements. This element type have the convenience of numerical implementation and also it can help to introduce $\mathrm{h} / \mathrm{p}$-refinement altogether. 


\section{Derivation of GIMP Shape Functions for 9-Node Lagrangian Grid Cell}

The 9-node Lagrangian GIMP functions can be easily derived with tensor product of the 1-dimensional case. So we start with 1D 3-node Lagrangian element. A general view of the grid which is formed by 1D 3-node Lagrangian elements is shown in figure 1. As typical in the GIMP instead of cell, shape function implementation is node based. Same figure also shows the material point with length $\left(l_{p}\right)$ and coordinate $\left(\boldsymbol{x}_{p}\right)$. The expressions for the 3-node Lagrangian grid shape functions are given as:

$$
\begin{array}{ll}
\psi_{1}(x)=\frac{2}{L^{2}}(L+x)(L / 2+x) & \text { for }-L \leq x \leq 0 \\
\psi_{2}(x)=\frac{4}{L^{2}}(L / 2-x)(L / 2+x) & \text { for }-L / 2 \leq x \leq L / 2 \\
\psi_{3}(x)=\frac{2}{L^{2}}(L-x)(L / 2-x) & \text { for } 0 \leq x \leq L
\end{array}
$$
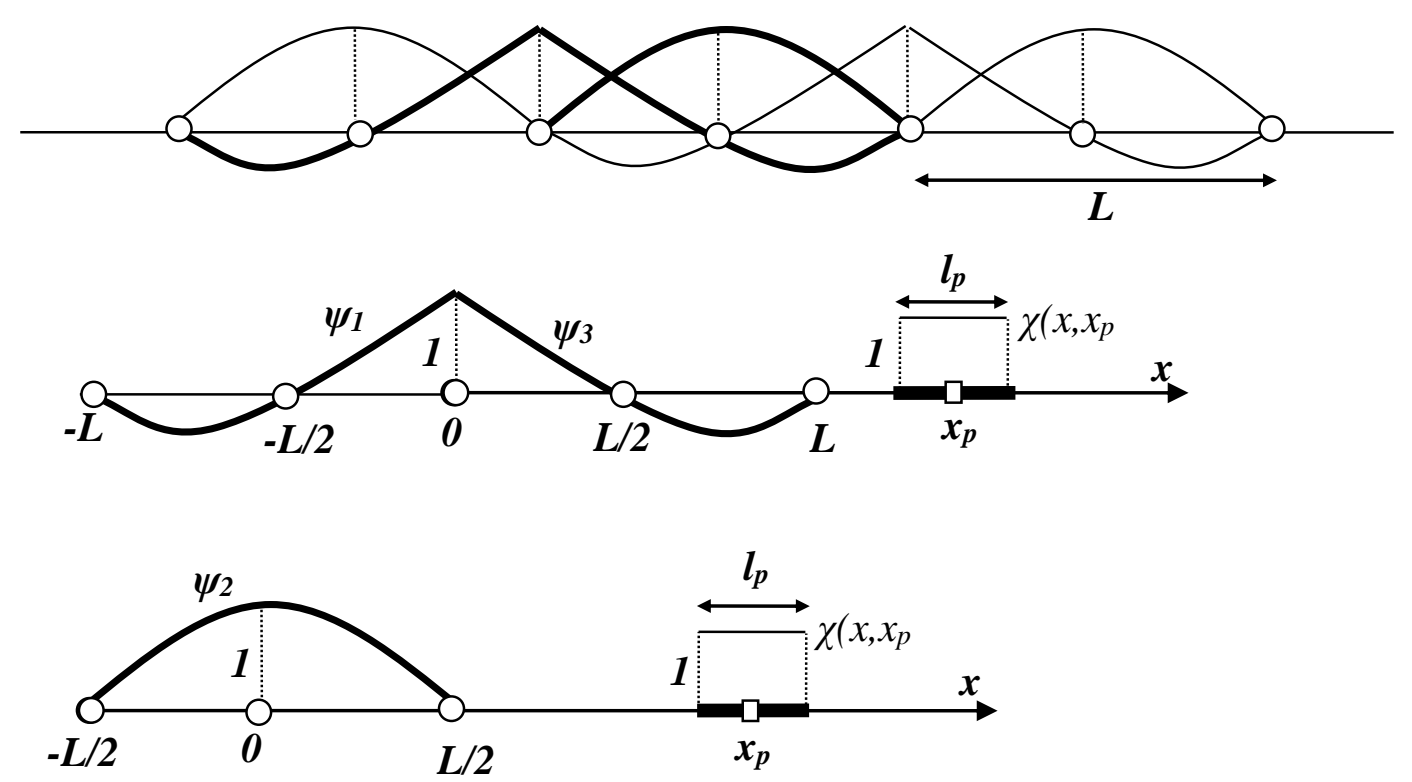

Figure 1: 3-Node Lagrangian Element Grid Shape Functions and Particle Characteristic Function

It is obvious that there are two different types of nodes: The nodes associated with grid shape functions 1,3 and the nodes associated with the grid shape function 2. Interaction of material points with these two types will be different. Let's denote the node with grid shape functions 1,3 with subscript $A$ and with grid shape function 2 with subscript $B$. In GIMP a characteristic function, $\chi\left(x, x_{p}\right)$, which defines the shape of the material point is used for the evaluation of the nodal GIMP functions. This is generally selected as a "hat" function:

$$
\chi\left(x, x_{p}\right)=H\left(x-\left(x_{p}-l_{p} / 2\right)\right)-H\left(x-\left(x_{p}+l_{p} / 2\right)\right)
$$


where $H(x)$ is the step function. The GIMP shape functions can be now easily calculated with the following integrals as described in [5]:

$\phi_{A}\left(x_{p}\right)=\frac{1}{l_{p}}\left(\int_{-L}^{0} \chi\left(x, x_{p}\right) \psi_{1}(x) d x+\int_{0}^{L} \chi\left(x, x_{p}\right) \psi_{3}(x) d x\right)$

$\phi_{B}\left(x_{p}\right)=\frac{1}{l_{p}} \int_{-L / 2}^{L / 2} \chi\left(x, x_{p}\right) \psi_{2}(x) d x$

Closed form expression for these integrals have complicated algebraic structures for any arbitrary value of the $\kappa=L / l_{p}$ ratio. In this study we will use integer ratios so for $\kappa=1,2,3, \ldots$ :

$\phi_{A}\left(\overline{x_{p}}\right)=\frac{\left(4-18 \kappa+24 \kappa^{2}+48 \kappa^{2}{\overline{x_{p}}}^{2}-72 \kappa^{3}{\overline{x_{p}}}^{2}\right)}{24 \kappa^{2}} \quad$ for $\quad \mathbf{0} \leq\left|\overline{x_{p}}\right| \leq \frac{\mathbf{1}}{2 \kappa}$

$\phi_{A}\left(\overline{x_{p}}\right)=\frac{\left(4+24 \kappa^{2}-72 \kappa^{2} \overline{x_{p}}+48 \kappa^{2}{\overline{x_{p}}}^{2}\right)}{24 \kappa^{2}} \quad$ for $\quad \frac{\mathbf{1}}{2 \kappa}<\left|\overline{x_{p}}\right| \leq \mathbf{1}-\frac{\mathbf{1}}{2 \kappa}$

$\phi_{A}\left(\overline{x_{p}}\right)=\frac{\left(2+9 \kappa+12 \kappa^{2}+4 \kappa^{3}-\left(12 \kappa+36 \kappa^{2}+24 \kappa^{3}\right) \overline{x_{p}}+\left(24 \kappa^{2}+36 \kappa^{3}\right) \overline{x_{p}}-16 \kappa^{3} \overline{x_{p}}\right)}{24 \kappa^{2}}$ for $\mathbf{1}-\frac{\mathbf{1}}{2 \boldsymbol{\kappa}}<\left|\overline{x_{p}}\right| \leq \mathbf{1}+$ $\frac{1}{2 \kappa}$

$\phi_{B}\left(\overline{x_{p}}\right)=\frac{\left(-2+6 \kappa^{2}-24 \kappa^{2}{\overline{x_{p}}}^{2}\right)}{6 \kappa^{2}} \quad$ for $\quad \mathbf{0} \leq\left|\overline{x_{p}}\right| \leq \frac{1}{2}\left(\mathbf{1}-\frac{\mathbf{1}}{\kappa}\right)$

$$
\phi_{B}\left(\overline{x_{p}}\right)=\frac{\left(-1+3 \kappa^{2}+2 \kappa^{3}+6 \kappa\left(1-\kappa^{2}\right) \overline{x_{p}}-12 \kappa^{2}{\overline{x_{p}}}^{2}+8 \kappa^{3}{\overline{x_{p}}}^{3}\right)}{6 \kappa^{2}} \quad \text { for } \quad \frac{\mathbf{1}}{\mathbf{2}}\left(\mathbf{1}-\frac{\mathbf{1}}{\kappa}\right)<\left|\overline{x_{p}}\right| \leq \frac{1}{2}\left(\mathbf{1}+\frac{\mathbf{1}}{\kappa}\right)
$$

where $\overline{x_{p}}=x_{p} / L$ is the dimensionless local coordinate of the particle with respect to the related node. Selecting the ratio $\kappa$ as integer directly dictates the number of material points per cell in the initial configuration so $\kappa=L / l_{p}$ is also particle per direction (PPD). It is obvious that in 1D particle per cell $(\mathrm{PPC})=$ PPD. Figure 2 depicts these new GIMP shape functions and their derivatives for $\kappa=2$. 

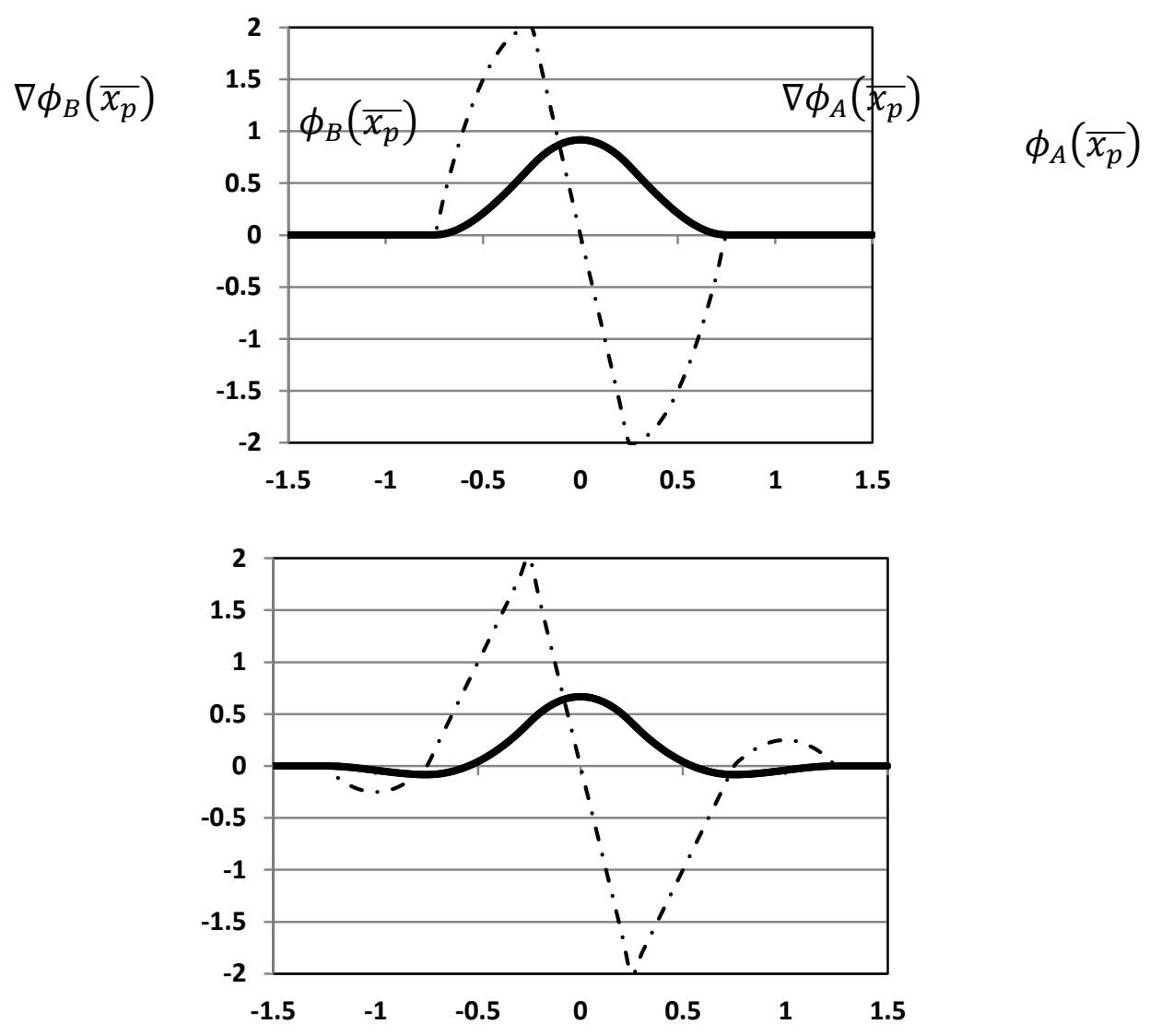

Figure 2: Developed 1D GIMP Shape Functions

It can be shown that partition of unity is satisfied. Here without a detailed proof we simply give the graphs of the new GIMP shape functions and their summation on the grid altogether for a series of adjacent nodes:

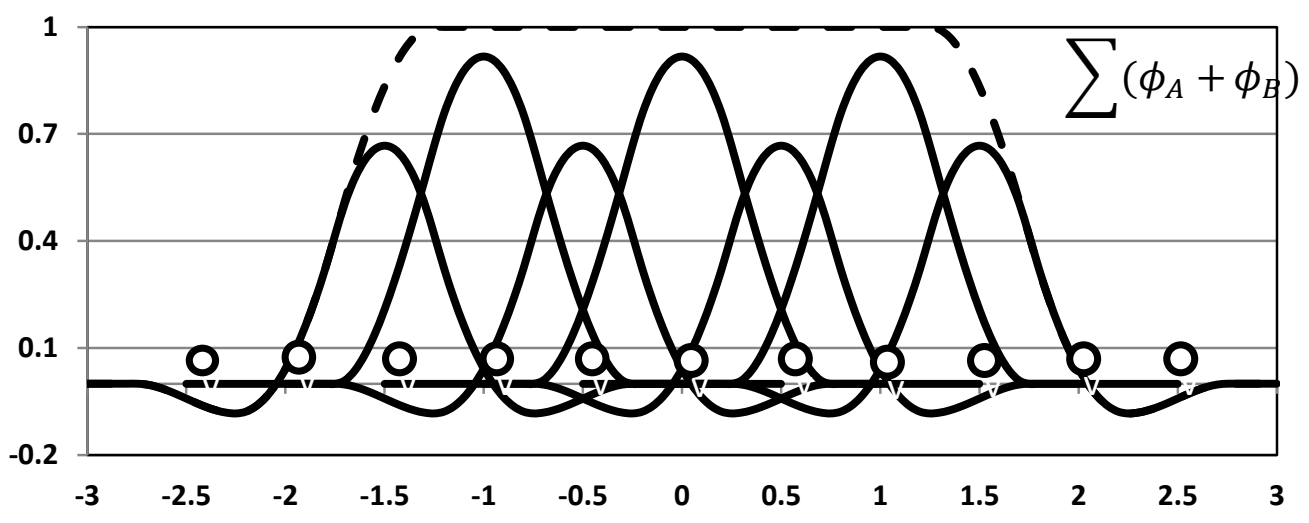

Figure 3: Partition of Unity Over Cell 
2D case can be readily obtained from 1D GIMP functions via tensor product. In this case there will 4 different points (AA,AB,BA,BB) on a regular mesh which is formed completely by 9 -node Lagrangian elements.

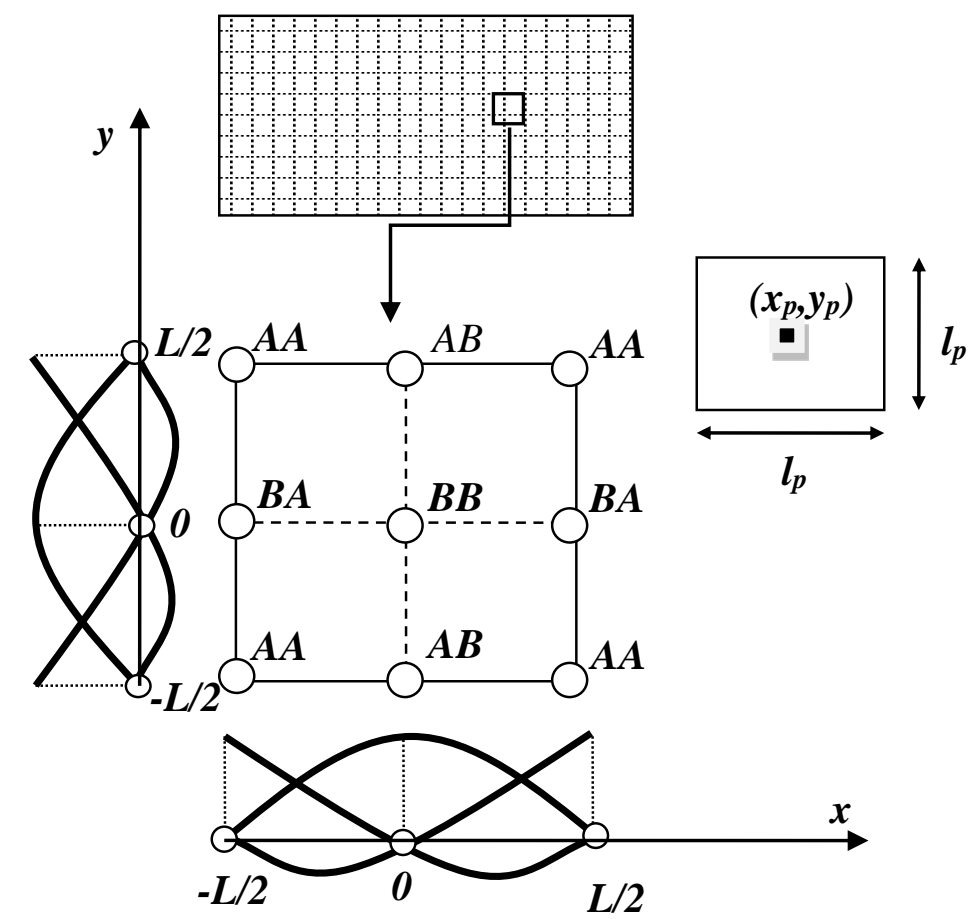

Figure 4: 2D Grid Shape Functions and Node Types

Using tensor product the GIMP functions $\theta_{\mathrm{AA}}, \theta_{\mathrm{BA}}, \theta_{\mathrm{AB}}, \theta_{\mathrm{BB}}$ for $2 \mathrm{D}$ case can simply expressed as:

$\theta_{A A}\left(x_{p}, y_{p}\right)=\phi_{A}\left(x_{p}\right) \phi_{A}\left(y_{p}\right)$

$\theta_{A B}\left(x_{p}, y_{p}\right)=\phi_{A}\left(x_{p}\right) \phi_{B}\left(y_{p}\right)$

$\theta_{B A}\left(x_{p}, y_{p}\right)=\phi_{A}\left(y_{p}\right) \phi_{B}\left(x_{p}\right)$

$\theta_{B B}\left(x_{p}, y_{p}\right)=\phi_{B}\left(x_{p}\right) \phi_{B}\left(y_{p}\right)$

Although not identical these functions have similar structures, in fact $\theta_{\mathrm{BA}}$ is $90^{\circ}$ rotated case of $\theta_{\mathrm{AB}}$ (they are symmetric with respect to $x=y$ ). Without detailed proof it can be shown that these 2D functions also satisfy partition of unity over the cell. In figure 5 they are depicted as surface plots for (particle per direction) $\mathrm{PPD}=2$ and thus (particle per cell) $\mathrm{PPC}=\mathrm{PPD}^{2}=4$ : 

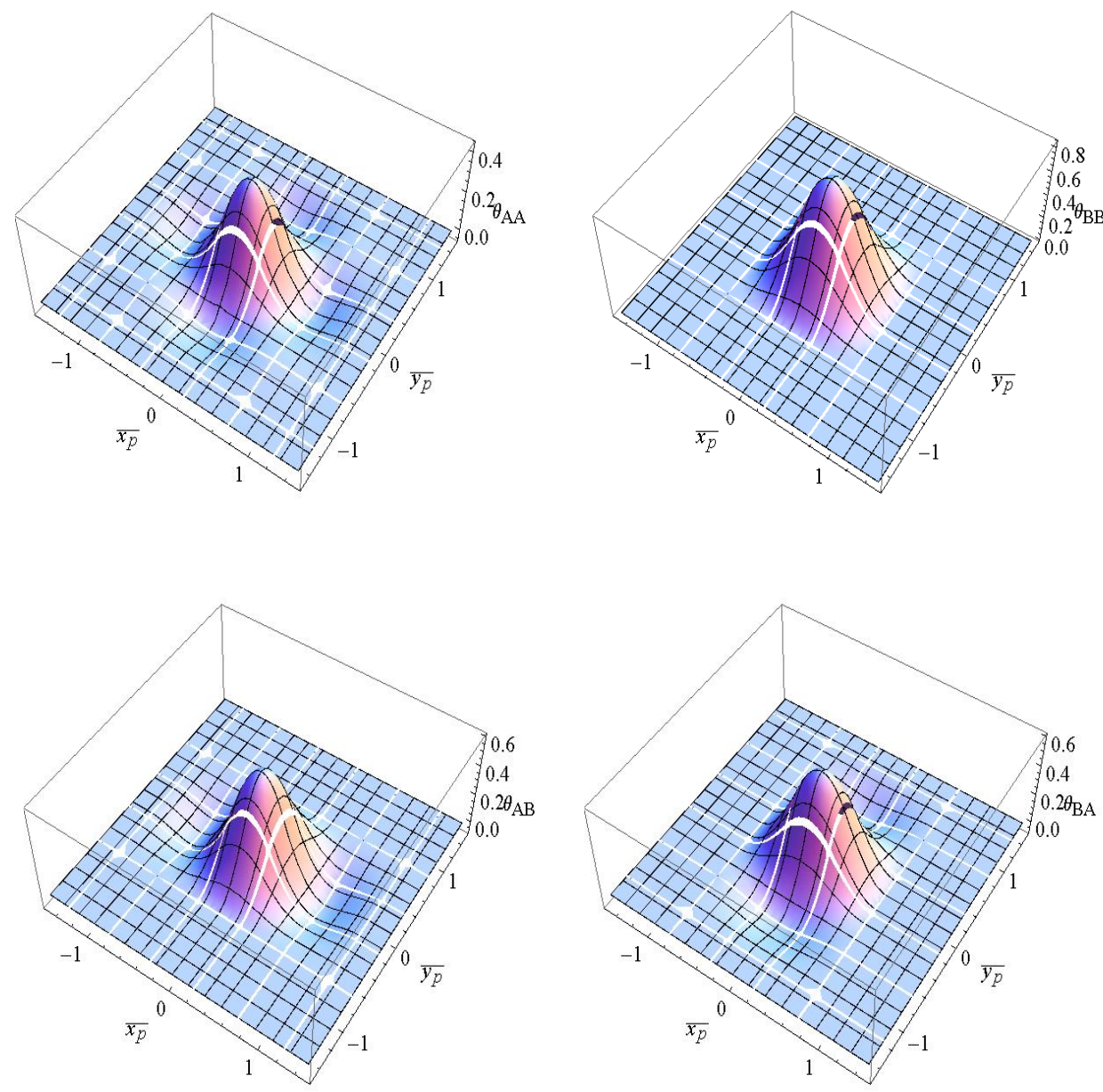

Figure 5: Developed 2D GIMP Shape Functions

\section{D Application w/ New GIMP Shape Functions: Shock Wave through Beam}

We started testing the new shape functions with a 1D application. The problem selected here is a cantilever beam under sudden loading. Figure 6 depicts the geometry and loading curve. Sudden loading causes a shock wave to appear and travel along the beam with a speed $(c)$ equal to $\sqrt{E / \rho}$ where A, $E$ and $\rho$ are area of cross section, Young's Modulus and density of the beam material respectively. 


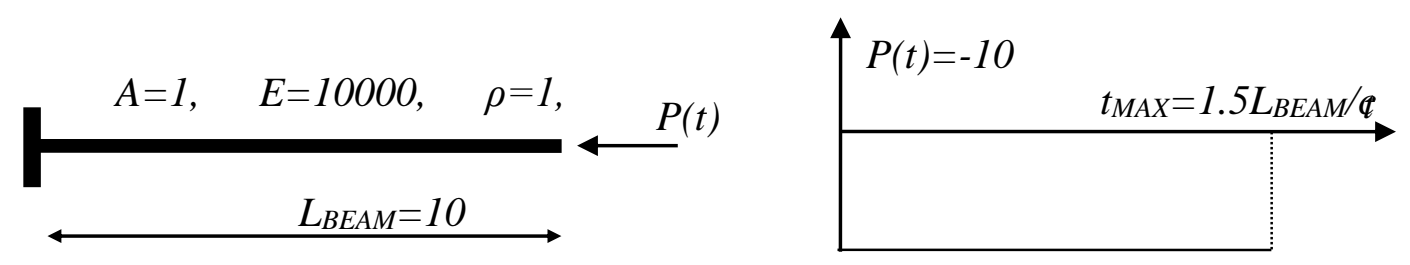

Figure 6: 1D Shock Wave Application Beam

The comparison method we use will be "ceteris paribus". There are two parameters: particle per cell $(P P C=1,2,4)$, resolution which is the grid spacing $(L=0.5,0.25,0.125)$. Courant number which is the ratio of the step time to the CFL time step $(\Delta t /(L / c)=0.5,0.25)$ is inherent to the $L$. Figure 7 shows the effect of changing PPC on the stress $\sigma$ at time $=t_{M A X}$ while $L=0.5$ and $C F L=0.25$ are held constant.

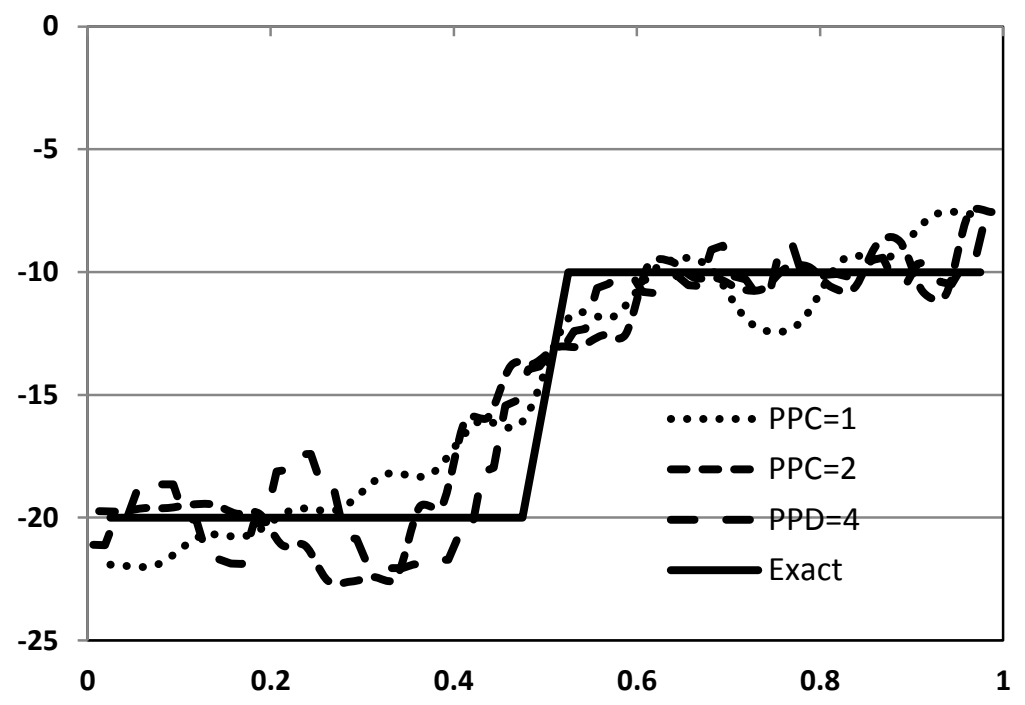

Figure 7: QGIMP Ceteris Paribus over $\sigma$ for $\mathrm{PPC}=1,2,4$

We can also calculate the $\mathrm{L}^{2}$ norm of error. Figure 8 shows this norm for $\mathrm{PPC}=1,2,4,8,16$

\begin{tabular}{|c|c|c|}
\hline PP & QGIMP & LGIMP \\
$\mathbf{C}$ & $\begin{array}{c}\text { Error Norm } \\
\mathbf{L}^{\mathbf{2}}\end{array}$ & Error Norm $\mathbf{L}^{\mathbf{3}}$ \\
\hline \hline 1 & 2.0585 & 2.2708 \\
\hline 2 & 2.0267 & 2.1497 \\
\hline
\end{tabular}




\begin{tabular}{|c|c|c|}
4 & 1.2287 & 2.1471 \\
\hline 8 & 1.1547 & 2.2446 \\
\hline 16 & 1.1533 & 2.3004 \\
\hline
\end{tabular}

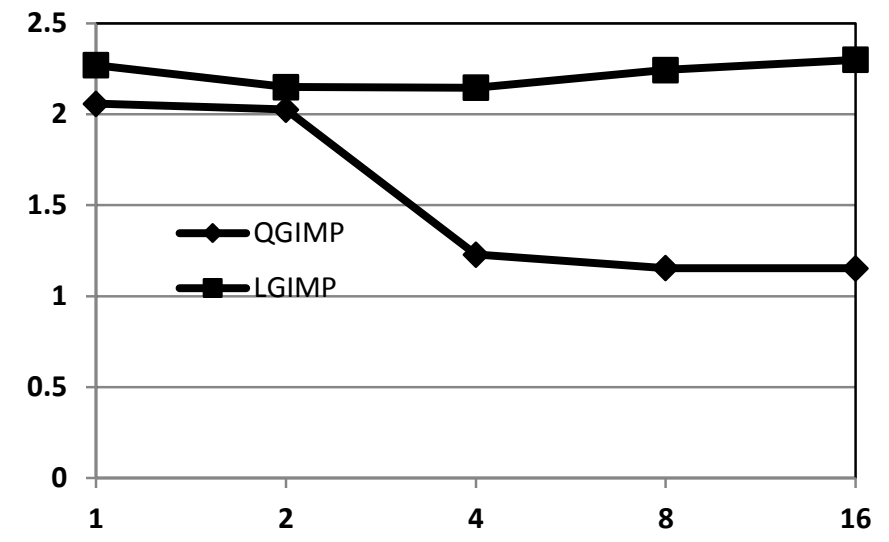

Figure 8: $\mathrm{L}^{2}$ Norm of Error vs. $\log _{2} \mathrm{PPC}$ and Error Table

As it is expected error reduces with increasing PPC. There is a sharp transition from $\mathrm{PPC}=2$ to $\mathrm{PPC}=4$ for QGIMP. Condition does not improve significantly for PPC $>4$. For the regular LGIMP condition worsens for PPC $>2$.

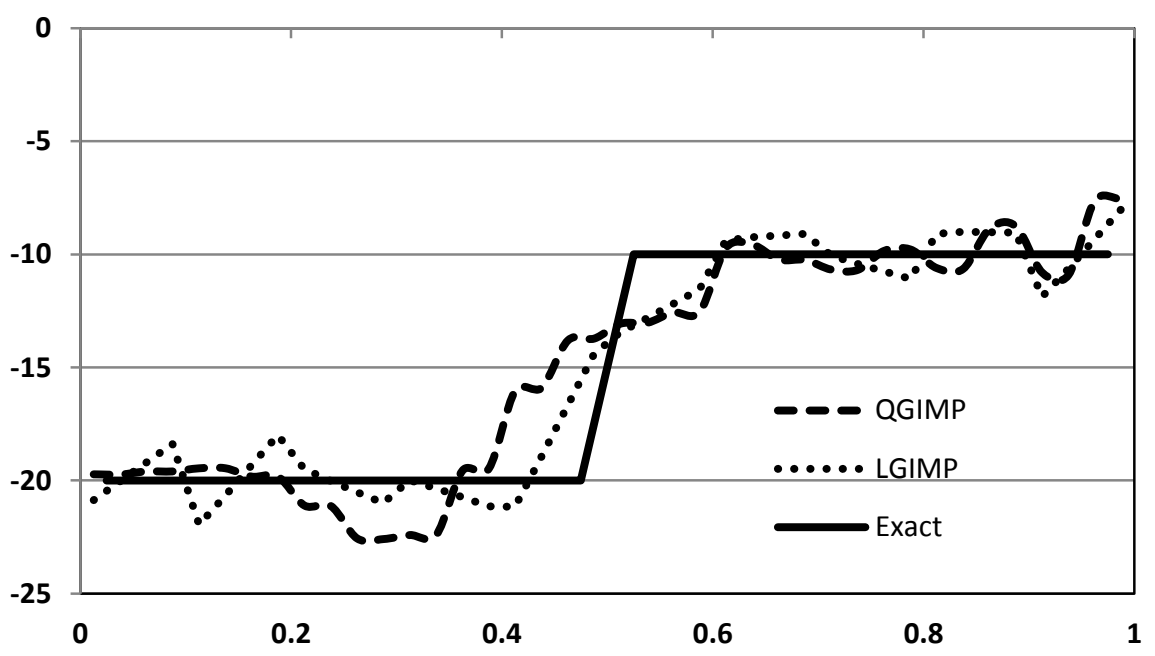

Figure 9: Comparison of $\sigma$ for QGIMP and LGIMP at time $=t_{\text {MAX }}$

In figure 9 we compared axial stress for QGIMP and LGIMP under same conditions: same number of nodes and same number of material points. QGIMP solution has 41 nodes and 40 particles, 
LGIMP solution has 40 nodes and 40 particles. In order make node numbers same resolutions are set to 0.5 and 0.25 for QGIMP and LGIMP respectively. Errors in this case become 2.026 and 2.032 for QGIMP and LGIMP respectively.

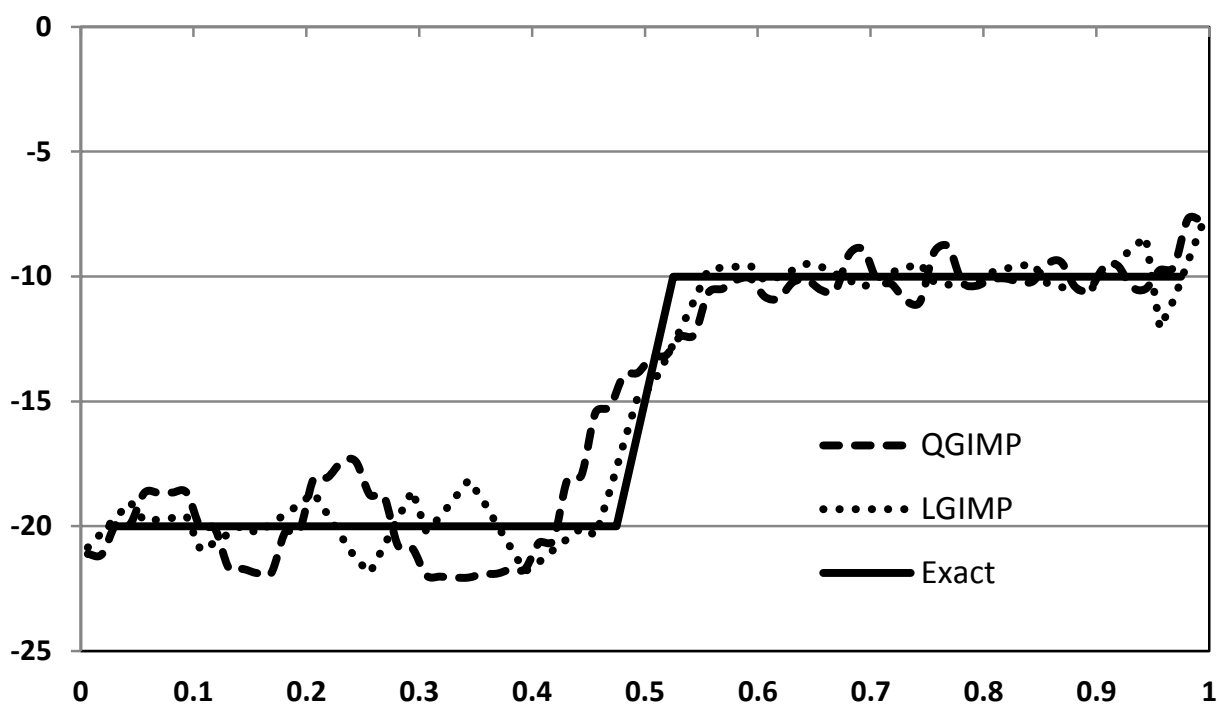

Figure 10: Comparison of $\sigma$ for QGIMP and LGIMP at time $=t_{M A X}$

Figure 10 repeats the same comparison for increased number of particles. 41 nodes and 80 particles for QGIMP. LGIMP solution uses 40 nodes and 80 particles. QGIMP clearly demonstrates its superiority with the error values 1.23 vs. 1.69 for LGIMP. It means that refining with QGIMP with increasing the order of the shape function (p-refinement) performs better than refining with LGIMP with decreasing cell length (h-refinement) .

When comparing resolution effect Courant number will also change. We take 0.5 for each simulation. Figure 11 and figure 12 shows the effect of resolution on axial stress $\sigma$ for $L=1,0.5,0.25$ when $\mathrm{PPC}=2$ and $\mathrm{PPC}=4$ respectively. 


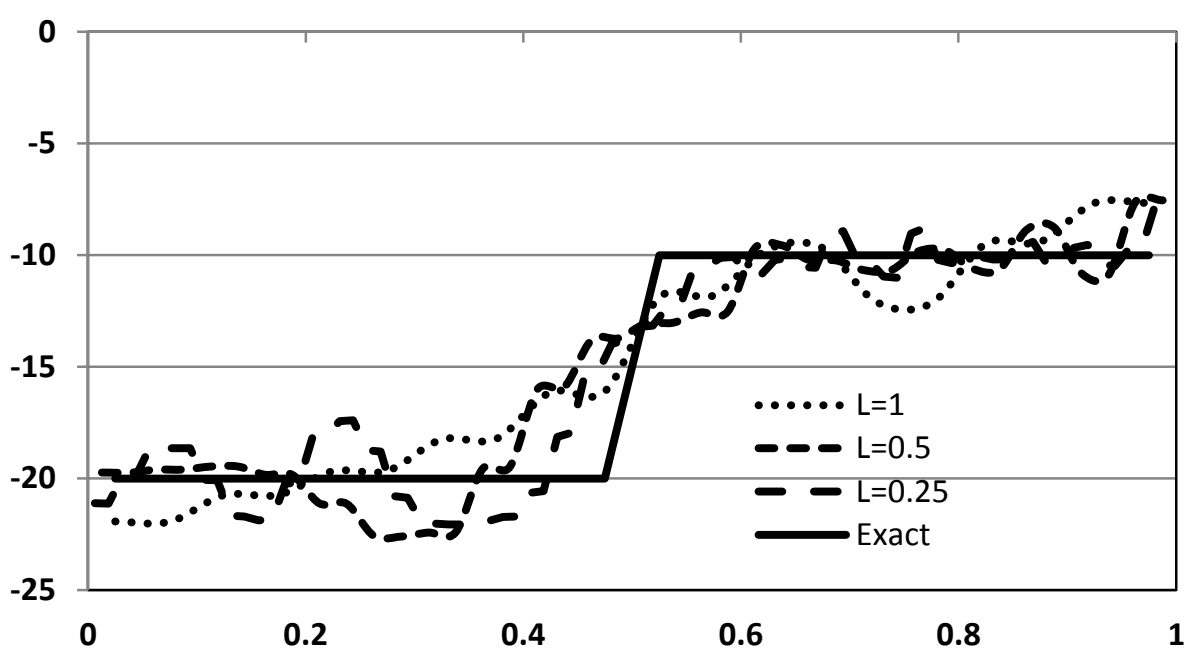

Figure 11: QGIMP Axial Stress at time $=\mathrm{t}_{\mathrm{MAX}}$ for $\mathrm{PPC}=2$, Courant Number $=0.5$

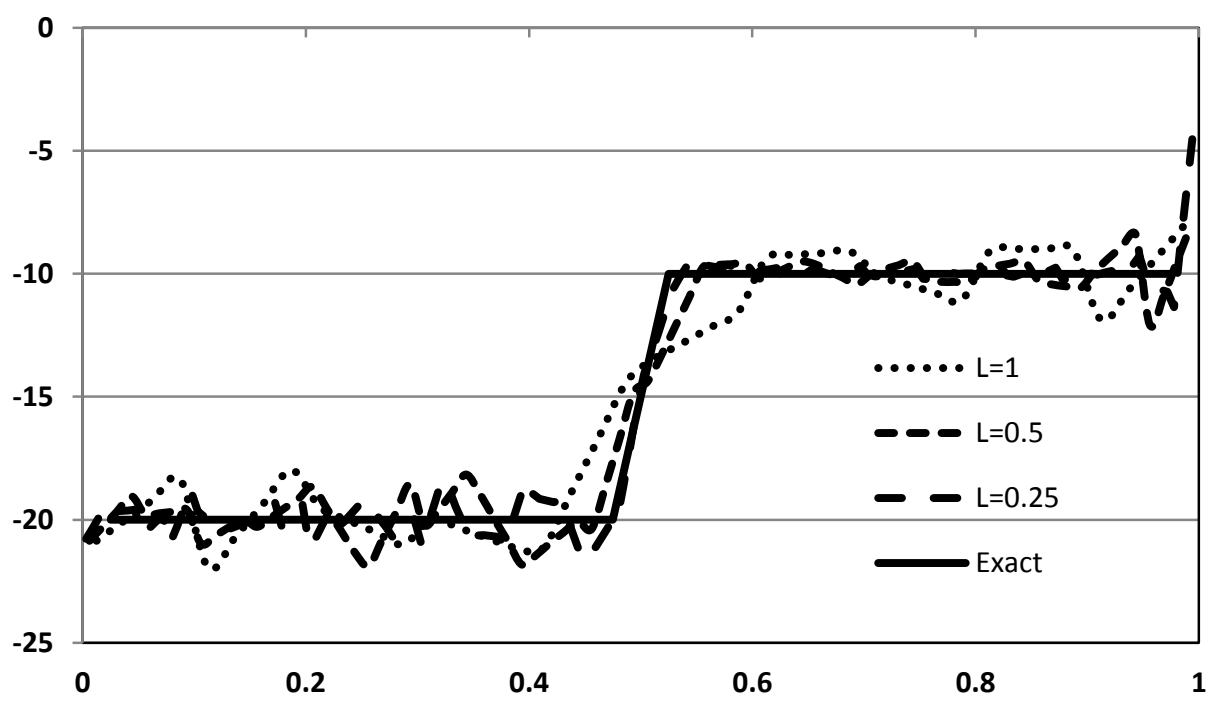

Figure 12: QGIMP Axial Stress at time $=\mathrm{t}_{\mathrm{MAX}}$ for $\mathrm{PPC}=4$, Courant Number $=0.5$

In figure 13 we gave the table for the $\mathrm{L}^{2}$ error norms of QGIMP and LGIMP solutions. The superiority of QGIMP over the LGIMP can clearly seen from the graph following the table. 


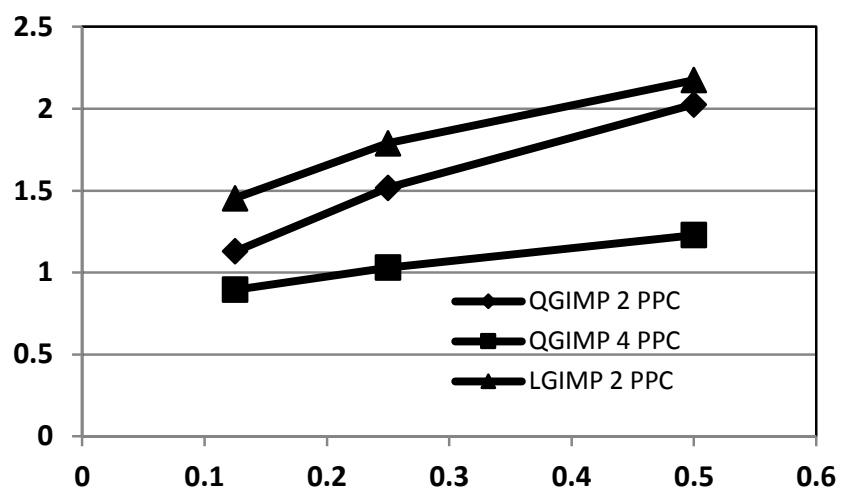

\begin{tabular}{|c|c|c|c|}
\multicolumn{2}{c}{ QGIMP } & QGIMP & LGIMP \\
\hline $\boldsymbol{L}$ & $\begin{array}{c}\text { PPC=2 Error } \\
\text { Norm } \mathbf{L}^{\mathbf{2}}\end{array}$ & PPC=4 Error Norm $\mathbf{~}^{\mathbf{2}}$ & PPC=2 Error Norm $\mathbf{L}^{\mathbf{2}}$ \\
\hline \hline 0.5 & 2.0267 & 1.2287 & 2.1748 \\
\hline 0.25 & 1.5180 & 1.0307 & 1.7902 \\
\hline 0.125 & 1.1294 & 0.8956 & 1.4538 \\
\hline
\end{tabular}

Figure 13: $\mathrm{L}^{2}$ Error Norm Comparison Graph and Table for QGIMP and HGIMP

\section{D Application w/ New GIMP Shape Functions: Shock Wave through Plate}

We selected this application because it is a natural extension to the 1D beam shock wave application. A square plate with unit thickness is used. Loading curve is same as in the 1D counterpart. Figure 14 depicts geometry.

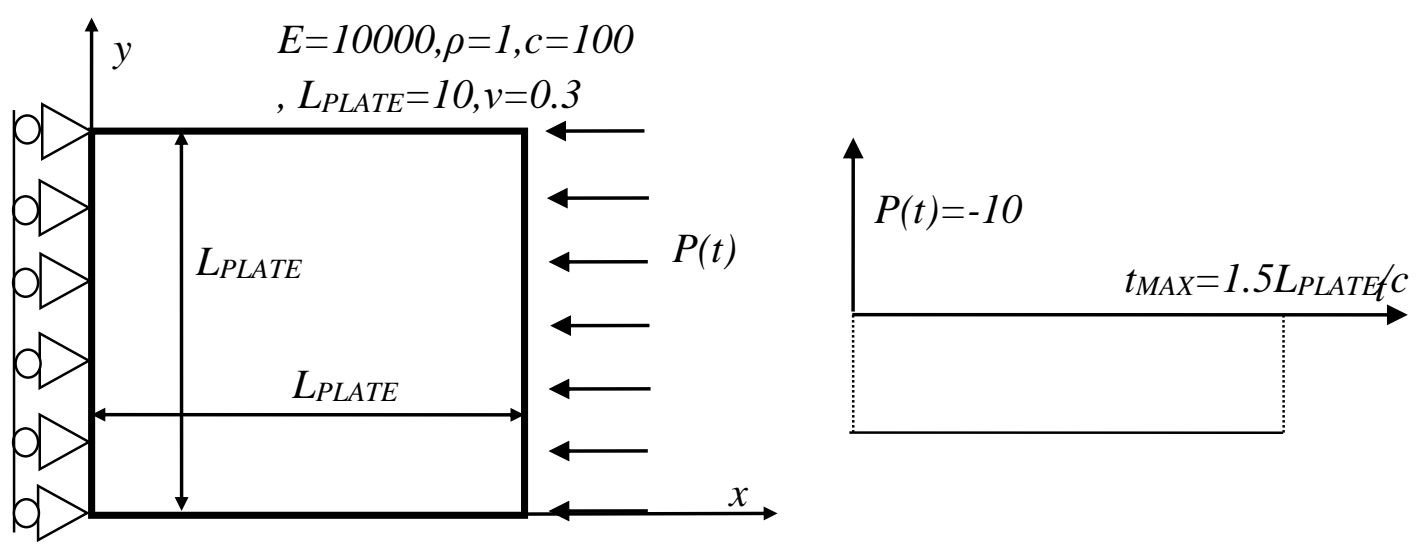

Figure 14: 2D Shock Wave Application Plate 
In figure 15,16 and 17 effect of number of PPC to the solution can be seen. As expected as PPC increases solution improves. For this simulations grid spacing $L=1$ (corresponds to 100 elements) is used. Courant number is always taken equal to 0.5 . The solutions are given for time $=\mathrm{t}_{\mathrm{MAX}}$. In figure 17 ABAQUS/EXPLICIT solution with 6400 elements is also given for comparison. Nonetheless using 100 elements is simply inadequate as it is obvious that even the $\mathrm{PPC}=25$ case cannot capture the details on ABAQUS solution.

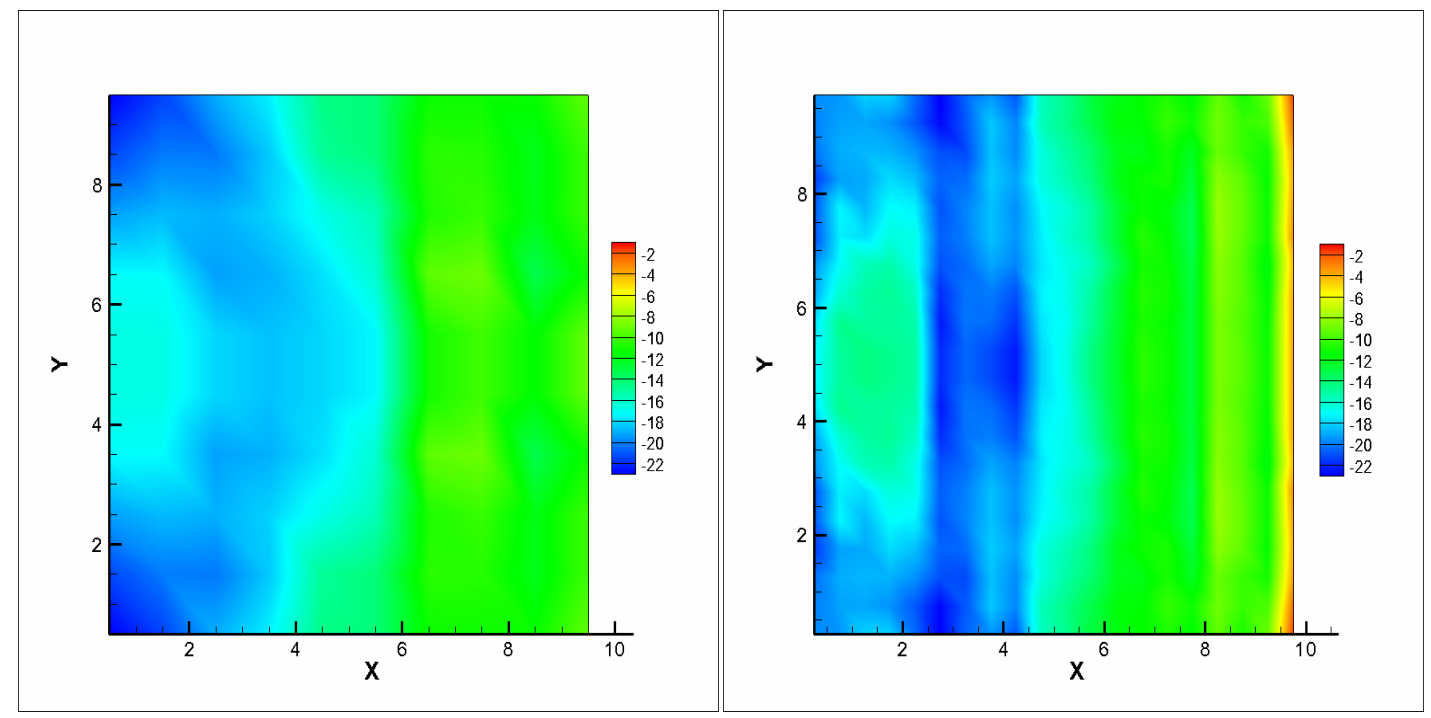

Figure 15: $\sigma_{X X}$ for $L=1$, Left $P P C=1$ and Right $P P C=4$

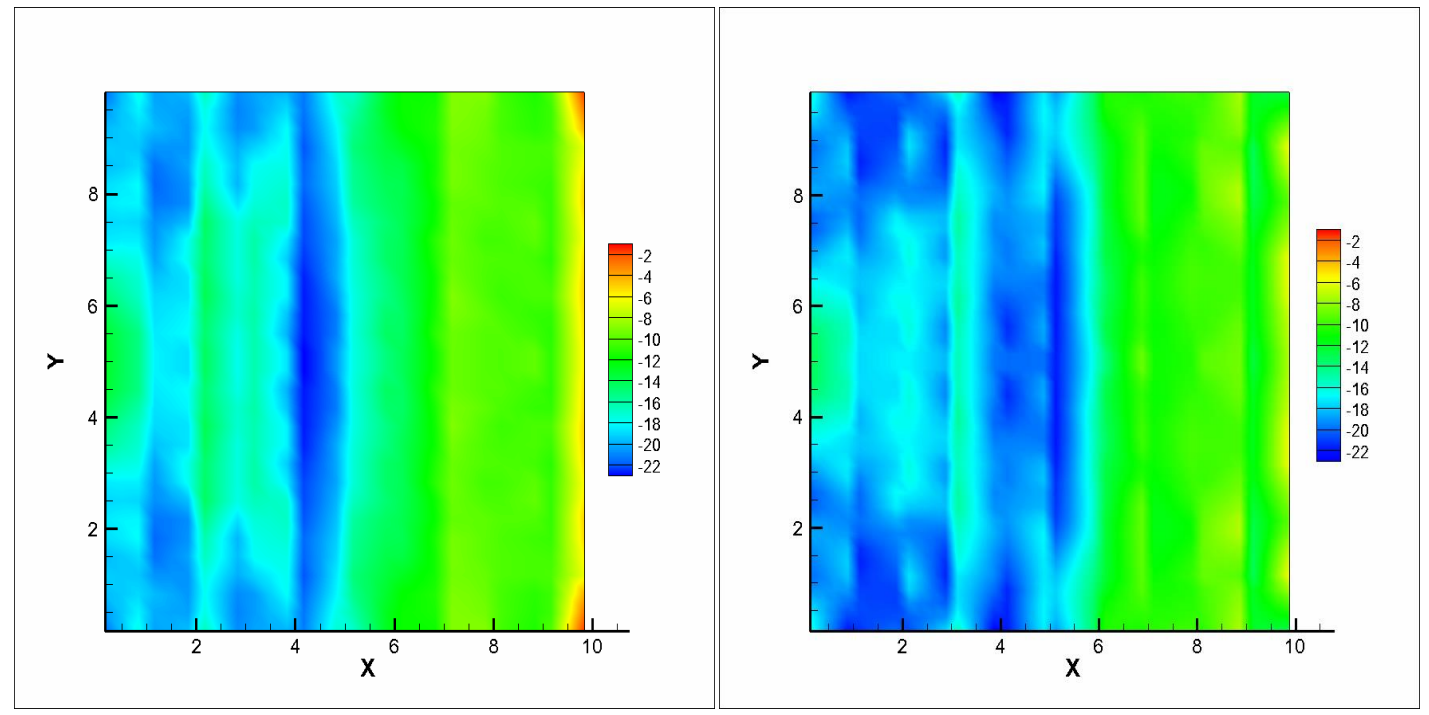

Figure 16: $\sigma_{X X}$ for $L=1$, Left $P P C=9$ and Right $P P C=16$ 

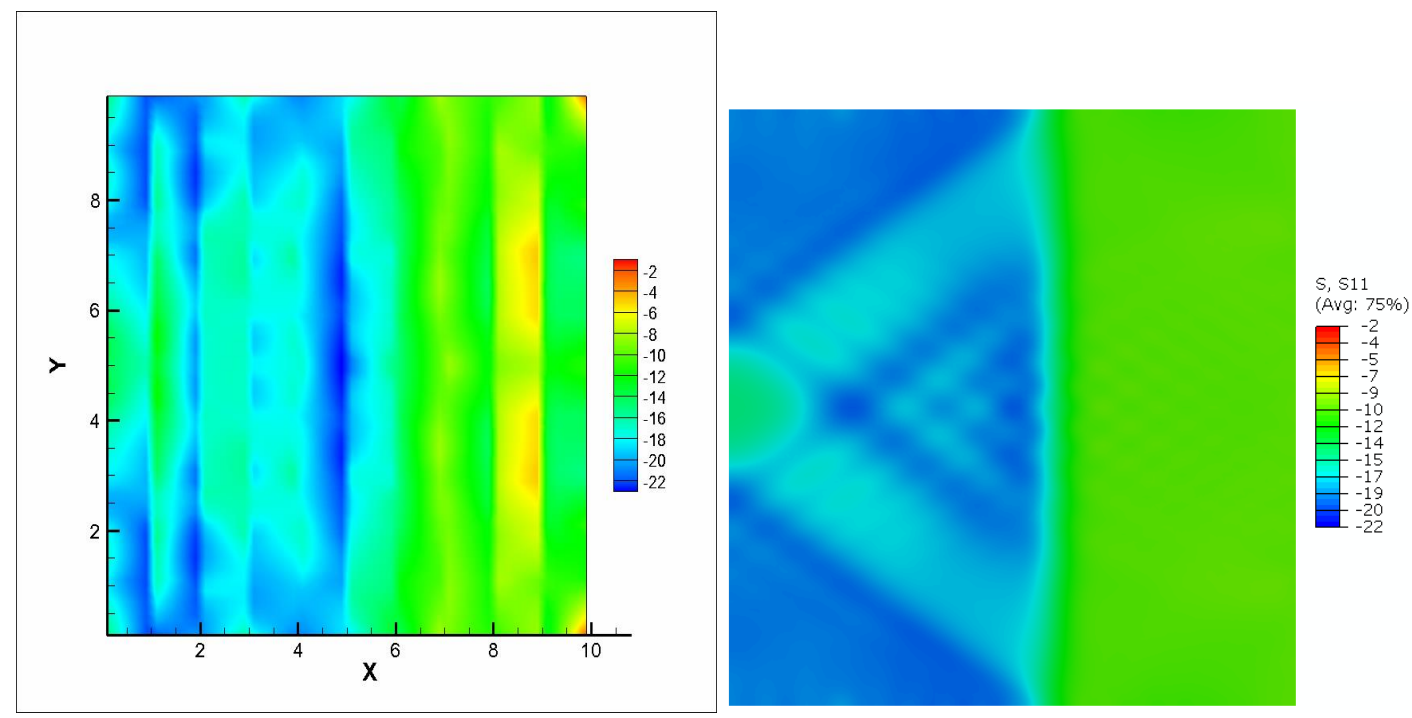

Figure 17: $\sigma_{X X}$ for Left $L=1$ (100 Elements), $P P C=25$ and Right ABAQUS/EXPLICIT 6400

Elements

In figure 18,19 and 20 we employed finer mesh. This time $L=0.5$ (400 elements). As expected solution improves with increasing PPC. This is valid up to $\mathrm{PPC}=16$. From $\mathrm{PPC}=16$ to $\mathrm{PPC}=25$ there is not any significant improvement. In the next page, figure $20, \mathrm{PPC}=25$ shows degradation due to particles begin to cross cell boundaries. This type of instability is the actual limitation of the QGIMP. Moreover PPC $=36$ could not complete the simulation because of this problem.

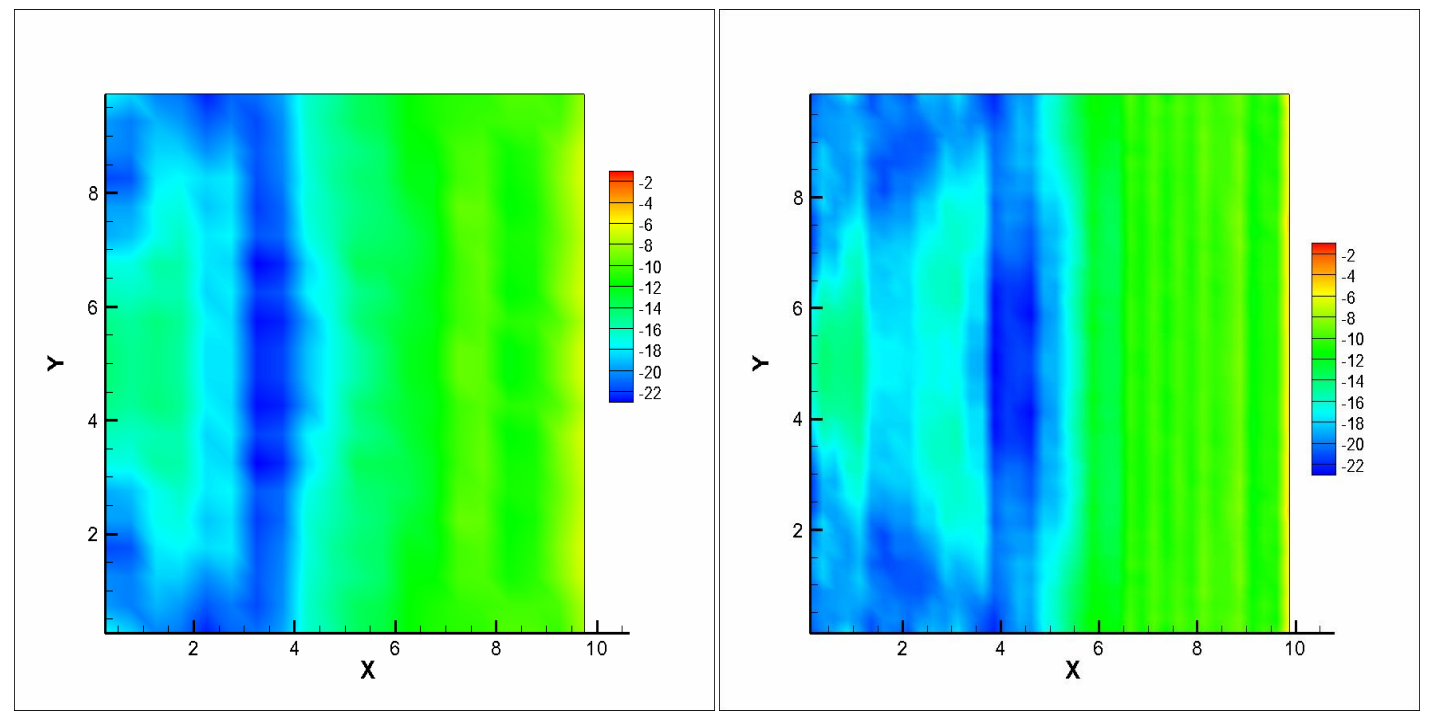

Figure 18: $\sigma_{X X}$ for $L=0.5$, Left $P P C=1$ and Right $P P C=4$ 


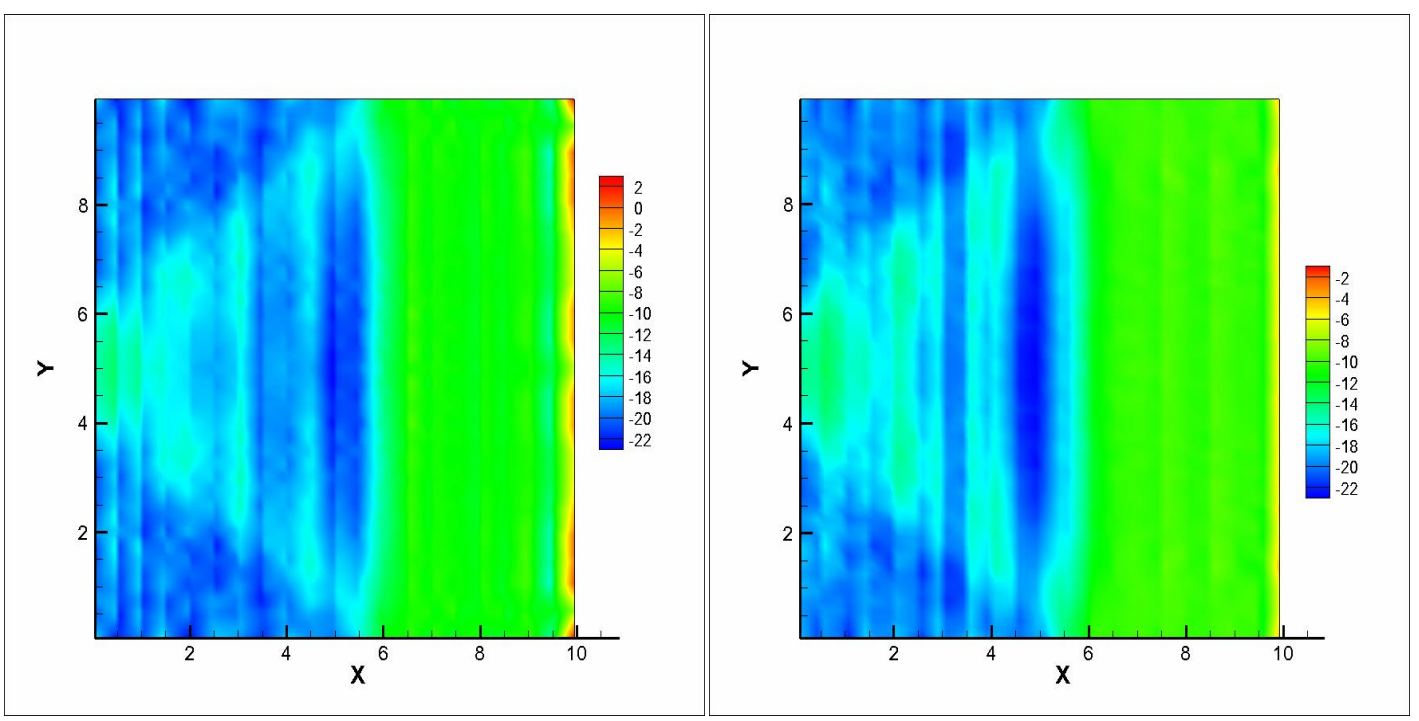

Figure 19: $\sigma_{X X}$ for $L=0.5$, Left $P P C=9$ and Right $P P C=16$
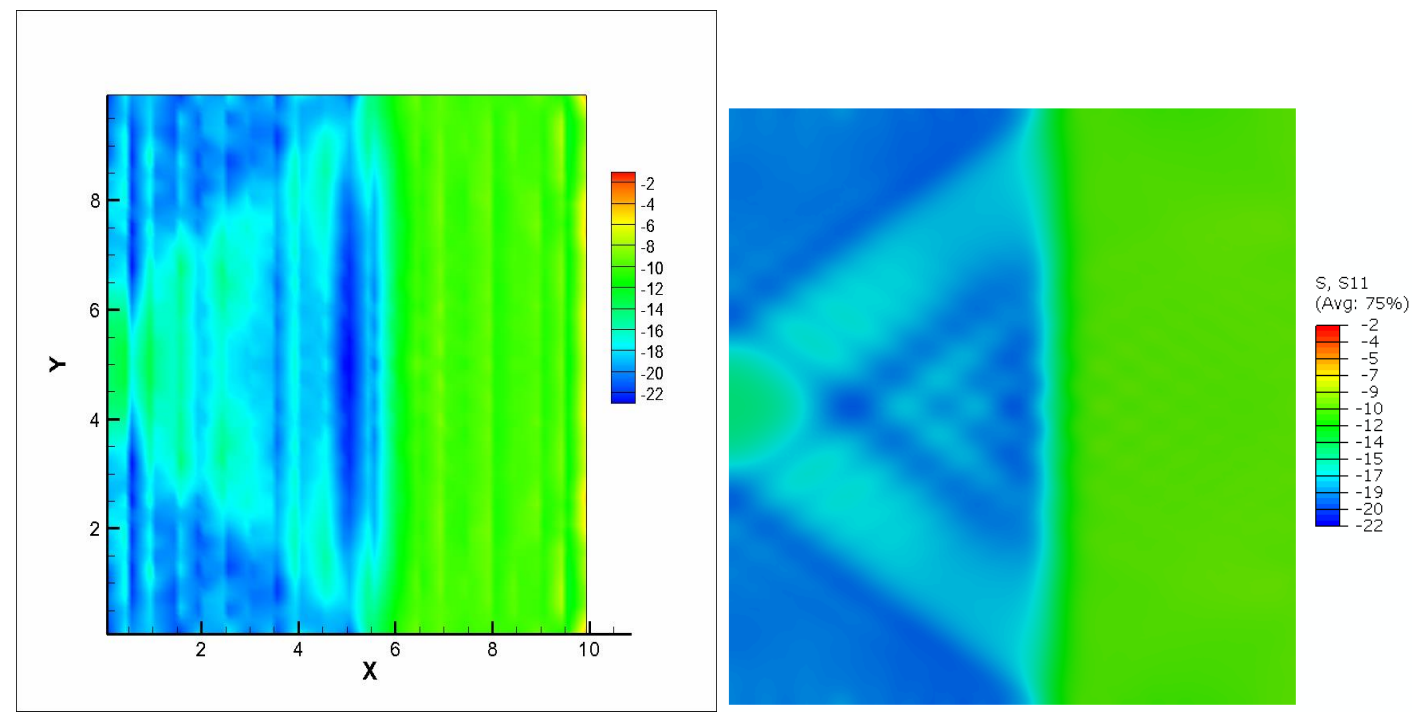

Figure 20: $\sigma_{X X}$ for Left $L=0.5$ (400 Elements), $P P C=25$ and Right ABAQUS/EXPLICIT 6400

Elements

In figure 21,22 and 23 we refined the mesh to the grid spacing of $L=0.25$ (1600 elements). This resulted in much better output. In figure 23 it can be seen that $P P C=25$ can capture almost all details of the corresponding ABAQUS/EXPLICIT solution with the quarter the number of elements. (1600 vs. 6400 for QGIMP and ABAQUS respectively). Finally in figure 24 effect of further refinement down to the size of $L=0.125$ (6400 elements) can be seen for $P P C=1$ and $P P C=4$. 


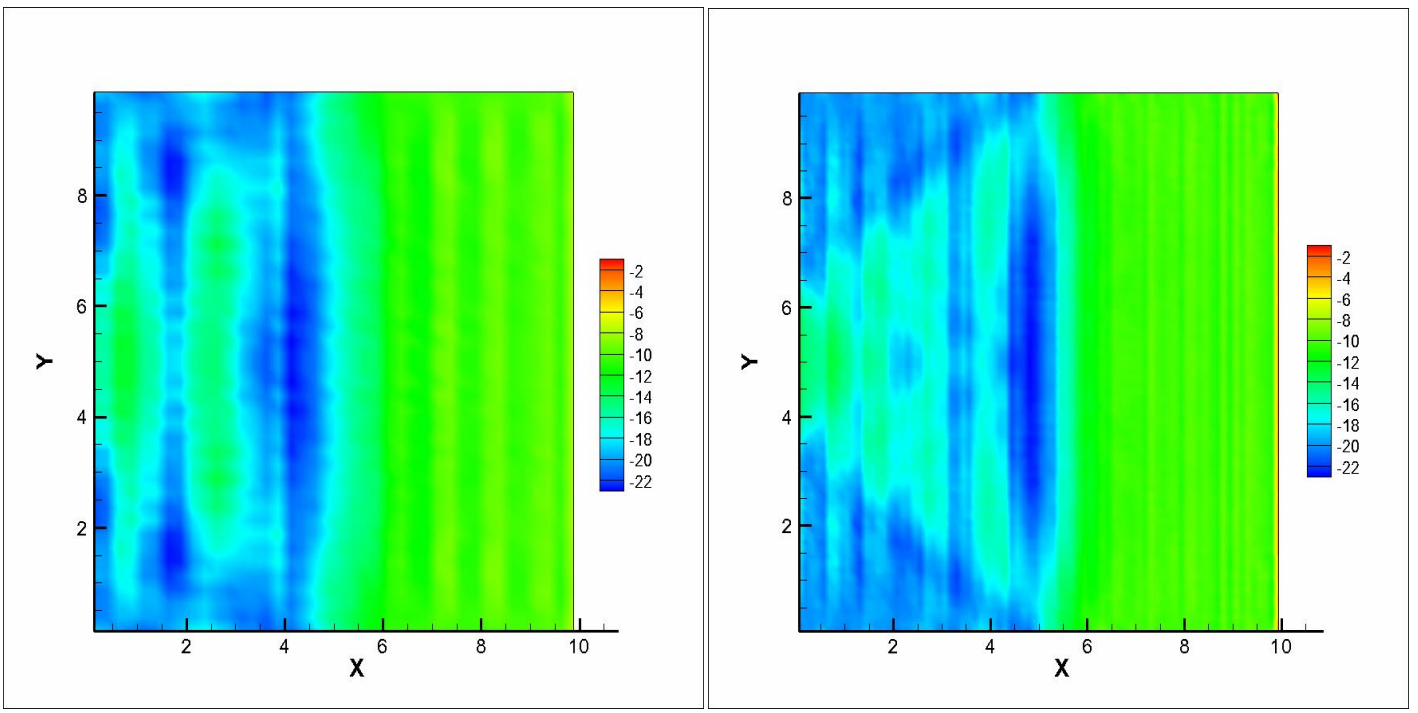

Figure 21: $\sigma_{X X}$ for $L=0.25$, Left $P P C=1$ and Right $P P C=4$

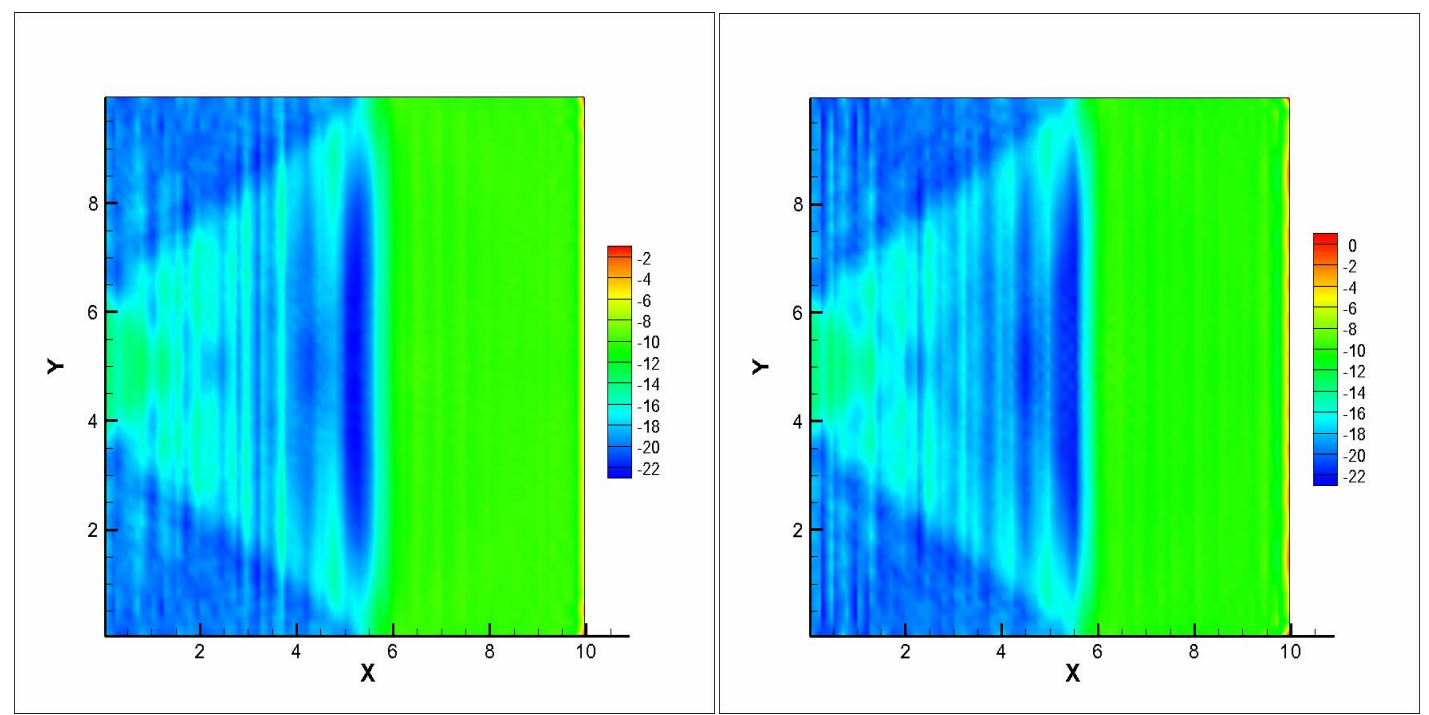

Figure 22: $\sigma_{X X}$ for $L=0.25$, Left $P P C=9$ and Right $P P C=16$ 

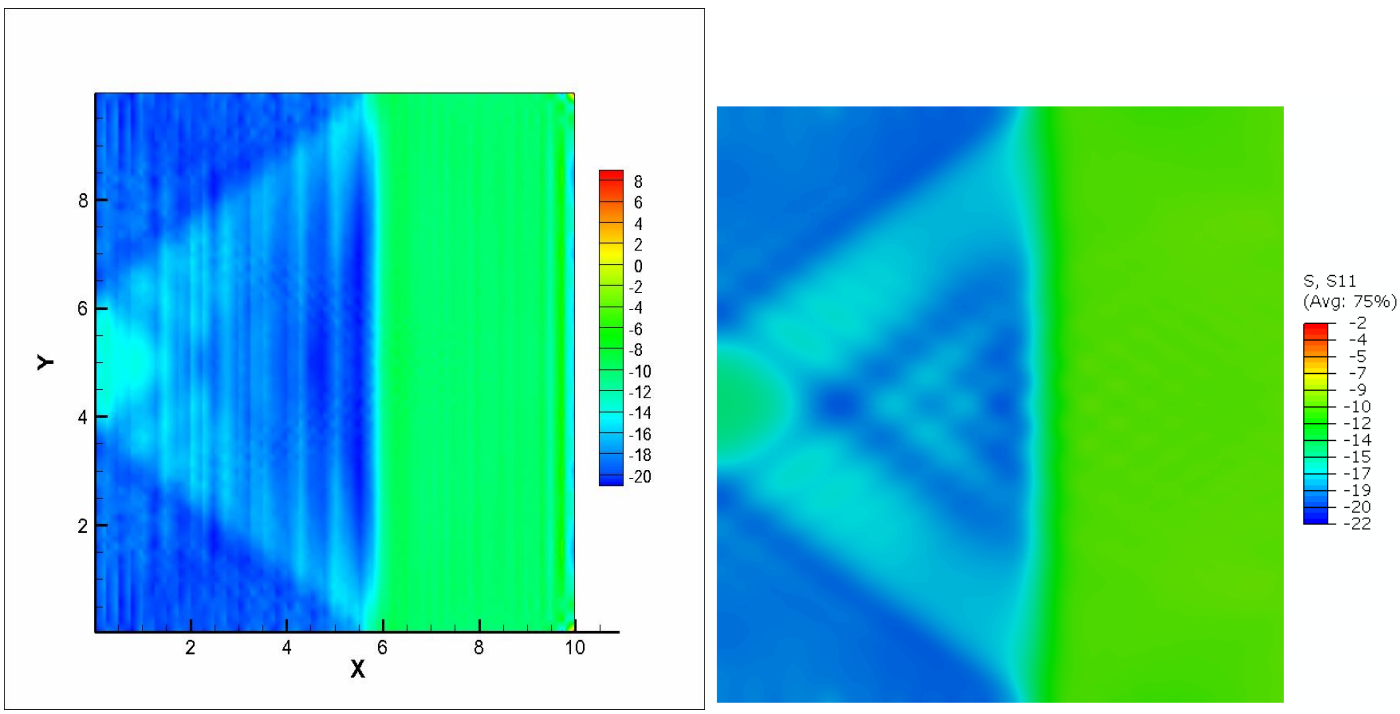

Figure 23: $\sigma_{X X}$ for $L=0.25$ (1600 Elements), $P P C=25$ Right ABAQUS/EXPLICIT (6400 elements)

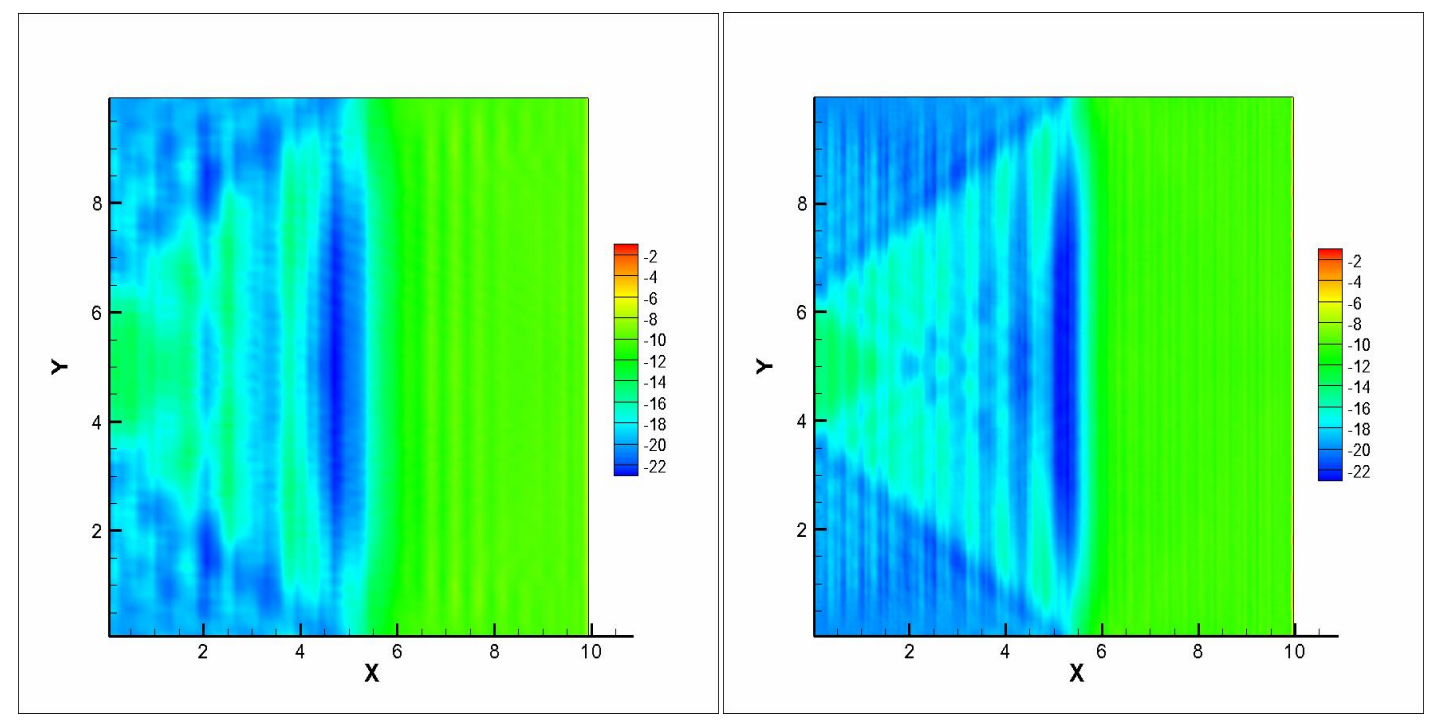

Figure 24: $\sigma_{X X}$ for $L=0.125$ (6400 elements), Left $P P C=1$ and Right $P P C=4$

A fair way to compare QGIMP and LGIMP would be comparing them under same conditions: same number of nodes and particles. In figure 25 and 26 grid spacing is set to $L=0.5$. A comparison between QGIMP and LGIMP is shown for $P P C=4$ and $P P C=16$ respectively. This means that there were about 1600 particles for $P P C=4$ and 6400 particles for $P P C=16$. The number of nodes is 1681 for both case. It is clear that QGIMP produces better results under same conditions. 


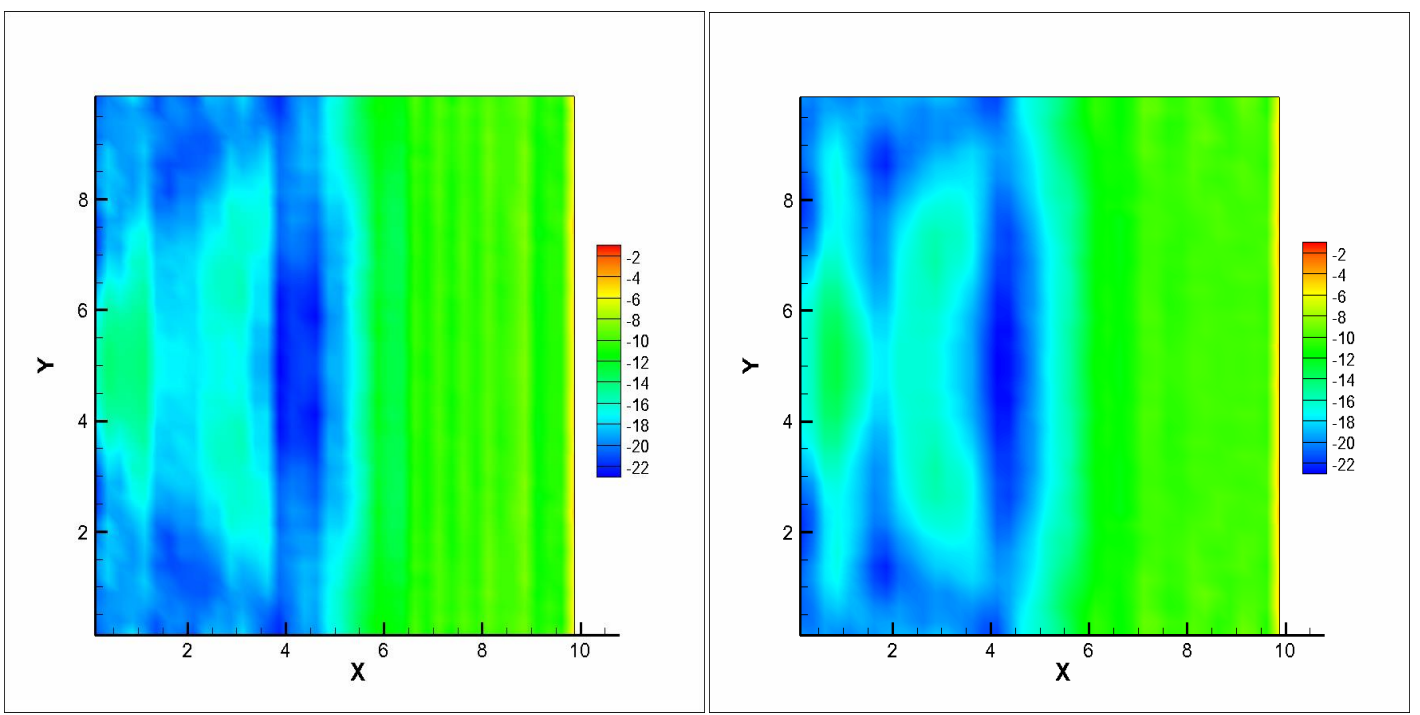

Figure 25: $\sigma_{X X}$ for Same Number of Nodes and Material Points $L=0.5, P P C=4$ Left QGIMP and Right LGIMP

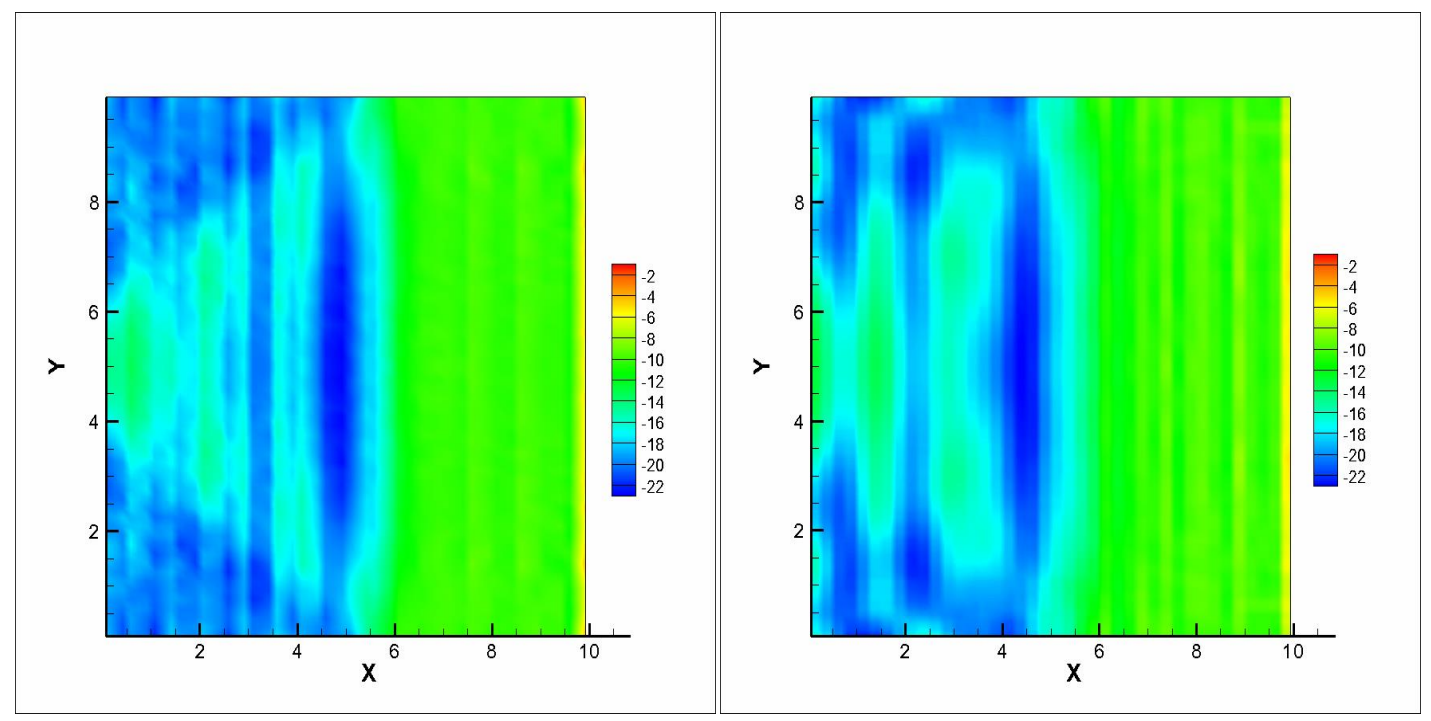

Figure 26: $\sigma_{X X}$ for Same Number of Nodes and Material Points $L=0.5, P P C=16$ Left QGIMP and Right LGIMP

Figure 27 and 28 is also another comparison between QGIMP and LGIMP. This time grid spacing is $L=0.25$. This means that there are 6561 nodes. Number of particles for $P P C=4$ and $P P C=16$ are 6400 and 25600 respectively. Again it is obvious that QGIMP is capturing much more detail than LGIMP under same conditions. 


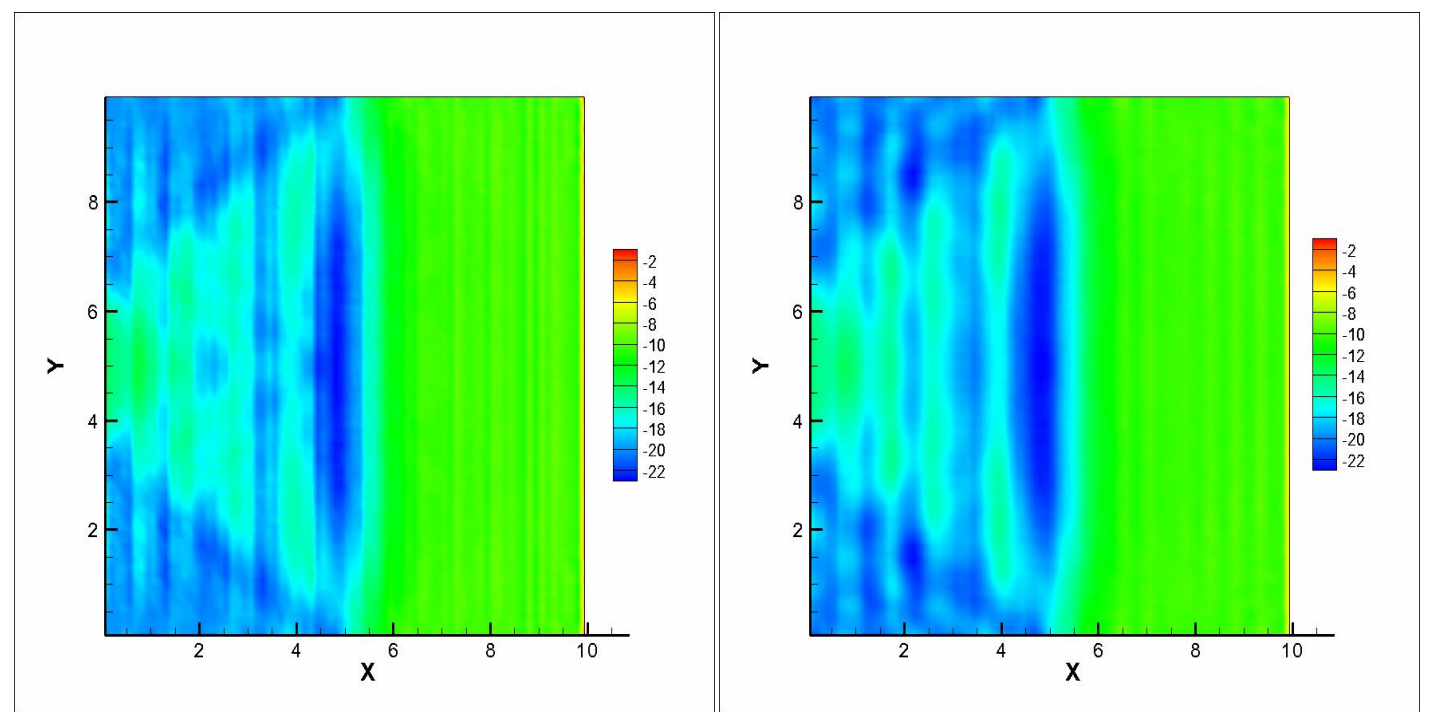

Figure 27: $\sigma_{X X}$ for Same Number of Nodes and Material Points $L=0.25, P P C=4$ Left QGIMP and Right LGIMP

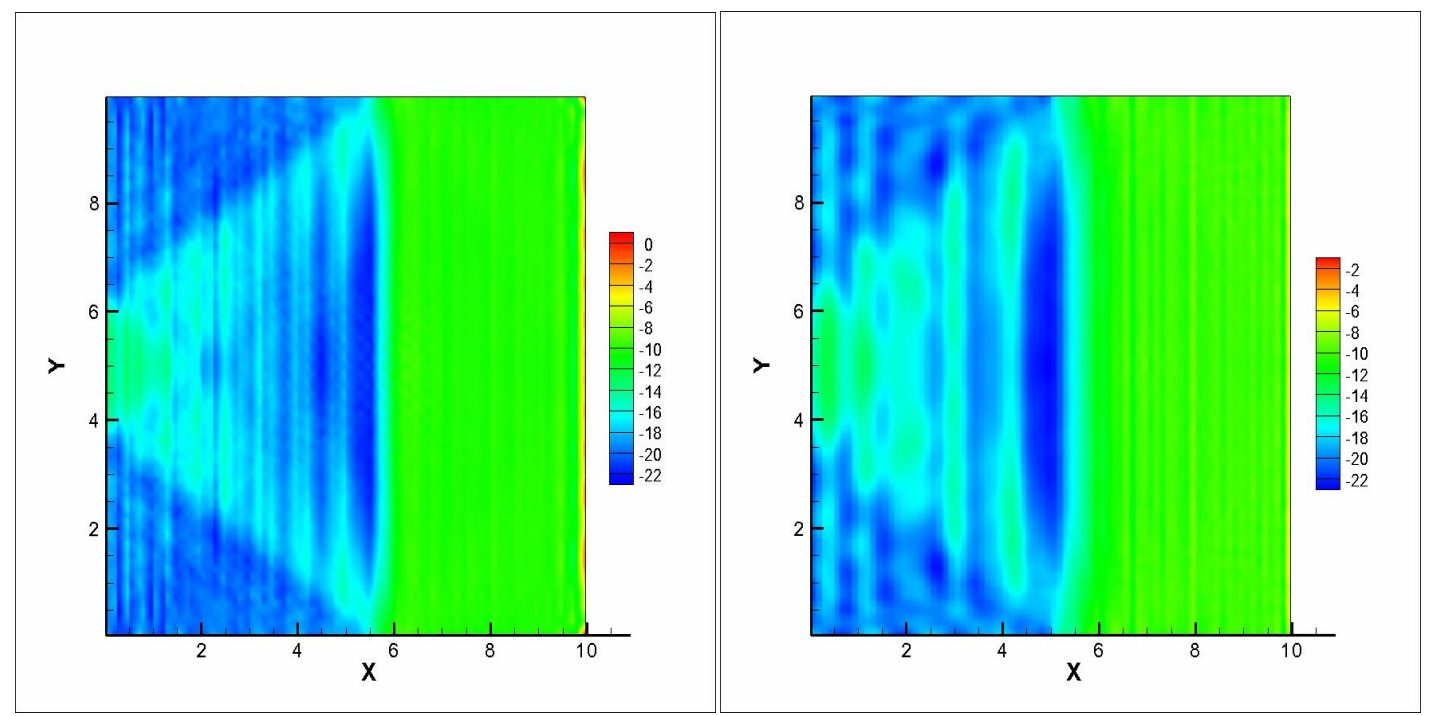

Figure 28: $\sigma_{X X}$ for Same Number of Nodes and Material Points $L=0.25, P P C=16$ Left QGIMP and Right LGIMP

As in the every particle method, especially when dealing with dynamic phenomena, there is noise in the solution. This sometimes can manifest itself as oscillation of the solution variables. The color shifts in the GIMP solutions are showing this fact. Thus the stress level in the GIMP solutions can not be as smooth as FEM when using a coarse mesh. Nonetheless it is obvious that they are getting smaller and smaller as we increase the resolution. 


\section{D Application w/ New GIMP Shape Functions: $K_{I}$ Stress Intensity Factor for DCB Specimen}

Another application we have selected is the calculation of the Mode I stress intensity factor $\left(\mathrm{K}_{\mathrm{I}}\right)$ of a double cantilever beam (DCB) specimen. As depicted in figure sudden load is applied to the DCB specimen. Quasi-static values of $\mathrm{K}_{\mathrm{I}}$ is calculated via introduction of artificial viscosity $(\mu=10)$.

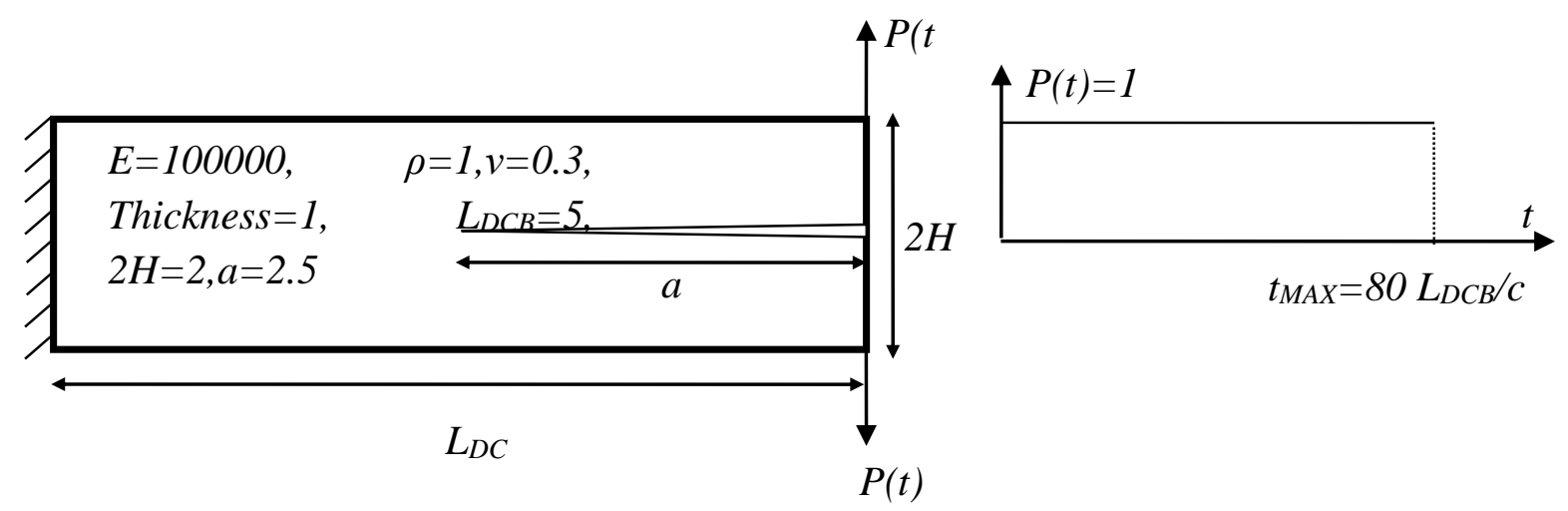

Figure 29: DCB Specimen Geometry and Loading

Analytical solution to this problem is given by [] as:

$K_{I}=2 P \sqrt{3}\left(\frac{a}{H}+0.64\right) / \sqrt{H}$

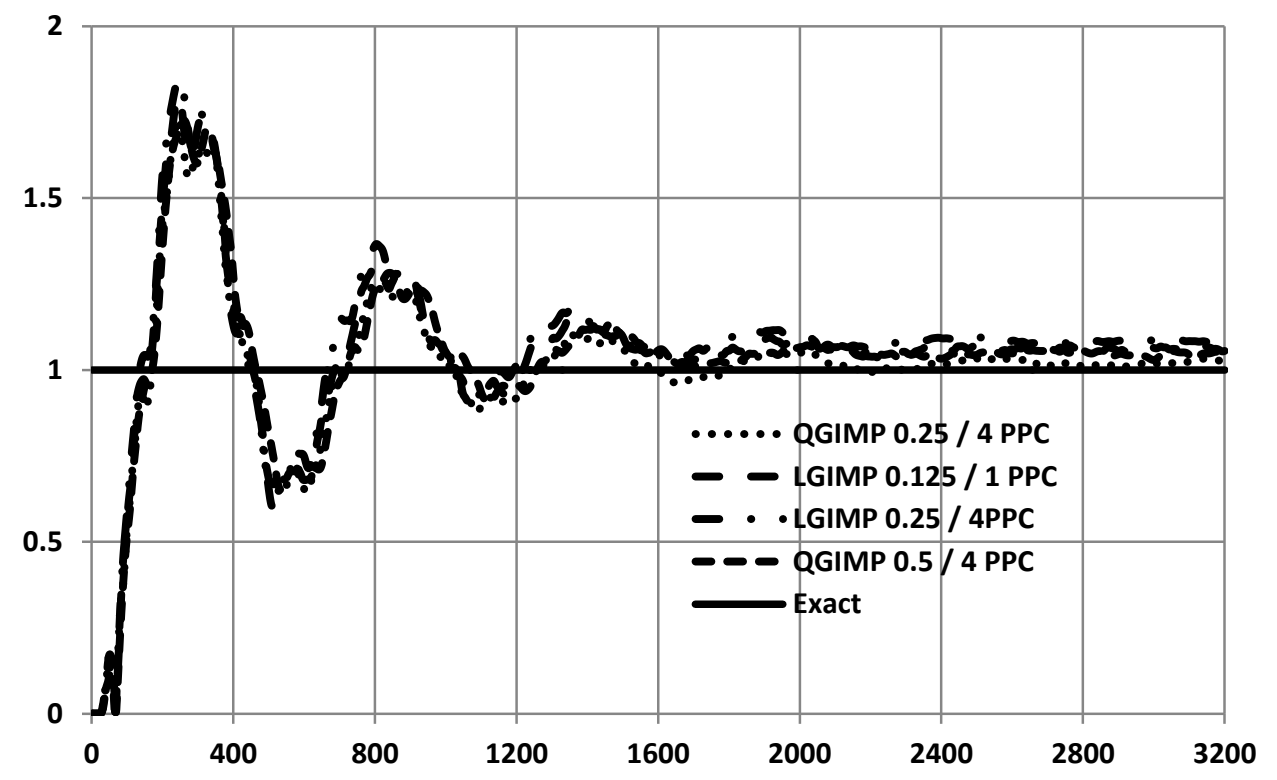


Figure 30: DCB Specimen Normalized Stress Intensity Factor vs. Step Number

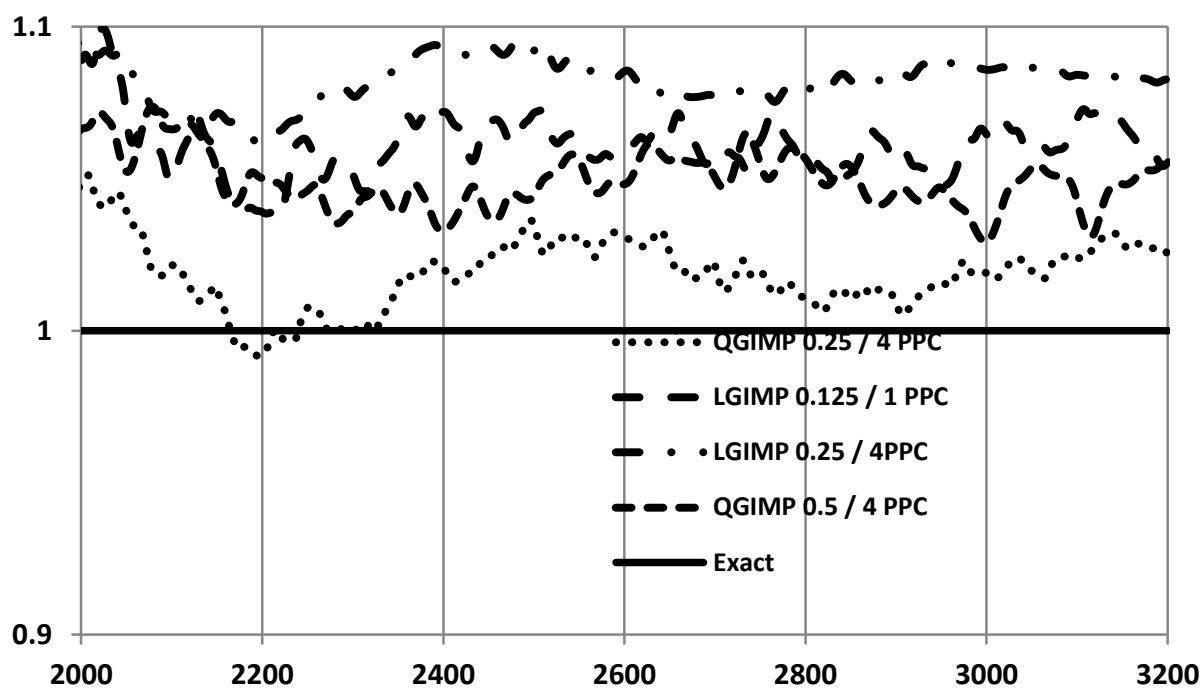

Figure 31: DCB Specimen Normalized Stress Intensity Factor vs. Step Number ZOOM

In figure 31 the steady state portion of figure 30 zoomed. This zoom clearly shows the superiority of QGIMP over LGIMP especially for stress concentration problems.

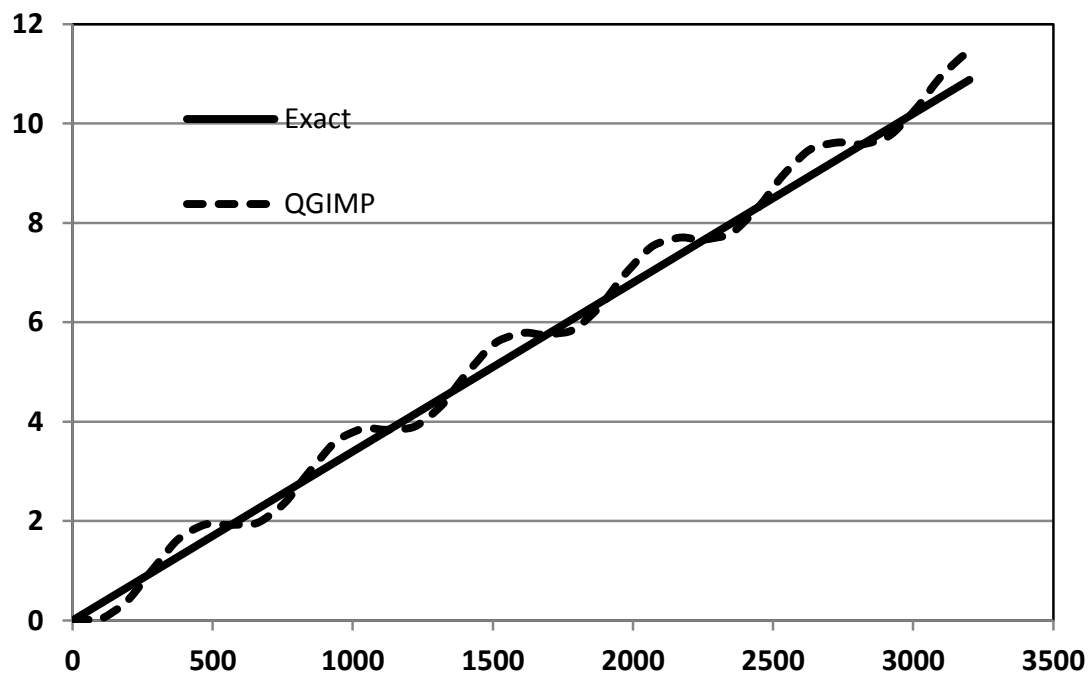

Figure 32: DCB Specimen Intensity Factor vs. Step Number

In figure 32 specimen is loaded with ramp loading. This is an alternate way of approximating the quasi-static case. As it is observed simulation follows the exact solution very closely for all load levels. 


\section{D Application w/ New GIMP Shape Functions: Stress Concentration around a Hole}

Our last problem is the classical hole in finite plate problem. In the numerical simulation plate and hole dimensions are taken as $L_{P L A T E}=10$ and $R=2$ respectively. Plate has unit thickness. The ratio $L_{P L A T E} / R=5$ qualifies as a close approximation to infinite plate case. The modeling of a hole on the structured grid which is formed by square cells is the biggest factor contributing to the error. In order model the curved boundary around hole high resolution should be used. Again a step loading is applied with artificial viscosity to ensure quasi-static stress state around the hole. Figure depicts the geometry and loading curve.

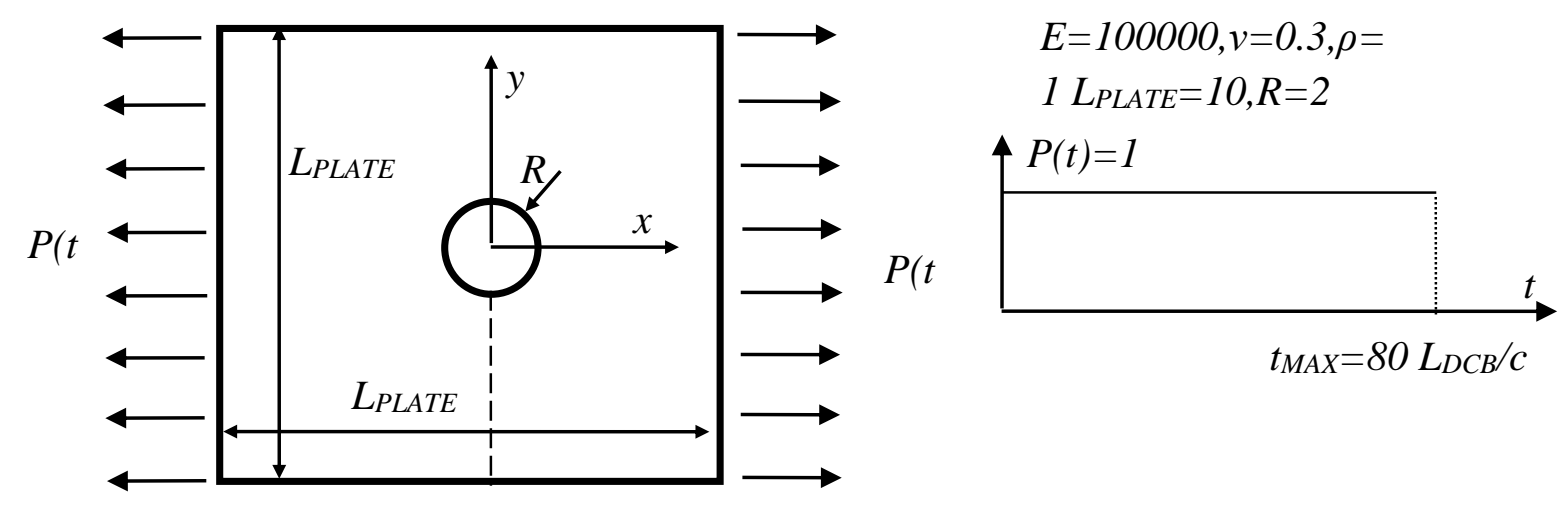

Figure 33: Hole in finite Plate Geometry and Loading

In figure 34 we plot $\sigma_{X X}$ along the dashed line in figure 33. Analytic solution for the infinite plate is well known:

$\left.\sigma_{x x}\right|_{x=0}=P\left(1+\frac{R^{2}}{2 y^{2}}+\frac{3 R^{4}}{2 y^{4}}\right)$ 


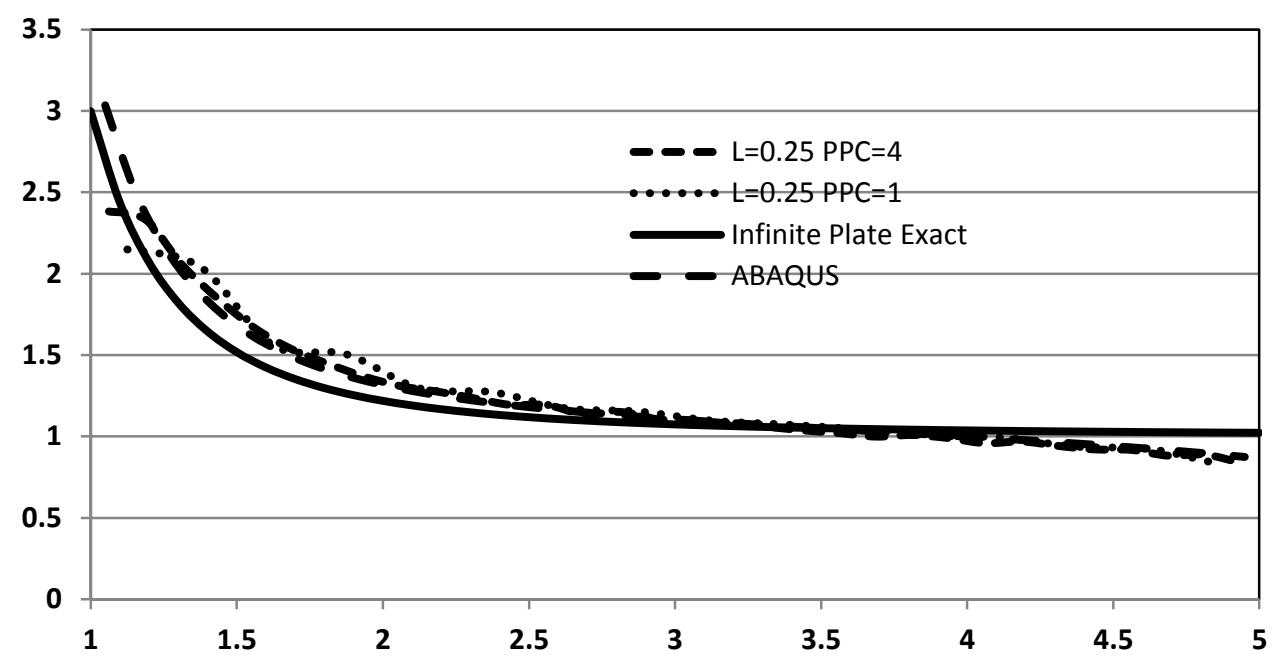

Figure 34: $L=0.25$ Hole in finite Plate $\sigma_{X X}$ at $x=0$.

There will be deviations from this solution since our problem includes a finite plate. In order to characterize this deviations better we have modeled the same problem with ABAQUS/STANDARD and this result is also plotted. It can be seen that the deviation at the edge of the plate is actually the effect of finite size. ABAQUS using 2256 elements is also capturing this. Deviation from ABAQUS is apparent at the hole boundary but this can be expected as ABAQUS uses quadrilateral mesh and fits better to the curved boundary. Moreover QGIMP uses 400 elements in contrast to ABAQUS's 2256 elements. Also it can be seen that as PPC increases from 1 to 4 solution also improves as expected. This has an combined effect: more material points means better approximation of integration (1) and better approximation of the curved boundary (2).

\section{Large Deformation Analysis}

Proposed QGIMP functions failed when the deformations were getting larger. There is not any sharp boundary between small and large deformations but we can accept that when particles begin to cross cell boundaries problems begin to arise. The most distinct feature of QGIMP is the availability of negative nodal masses. This is obvious from the shape functions from figure 2 and 5. When a particle is in the negative support region it will map negative nodal values to the related node. For some configuration of particles on the grid negative and positive masses can cancel each other and total mass can be zero on associated node. This is completely different from the LGIMP where when nodal mass is zero then it means there is not any particle in the support of that node and that node can simply be excluded from the simulation because it is no longer occupied. In QGIMP there are particles in the support but they render nodal value to zero. The node is still occupied so it cannot be discarded. This causes division by zero or very small values (since there is finite precision with float operations nodal value is never equal to exact zero). This will clearly introduces the instability. 


\section{Conclusion}

A new set of GIMP functions with quadratic grid functions are derived. This new frame is called QGIMP as opposed to the previous regular GIMP which was derived with linear grid functions hence called LGIMP here. From the selected problems it is shown that QGIMP demonstrated superior convergence properties under same conditions (same number of nodes and particles). Unfortunately when deformations are getting larger (particles begin to cross cell boundaries) then instabilities begin to occur. In this format QGIMP is not suitable for large deformation analysis. However application of some novel particle deformations tracking strategies [6] one may be able to increase the survivability in large deformation regime. 


\subsection{Failure Pattern Analysis of Bulk Failure in Cladding}

\section{Mechanical Properties of T91 Stainless Steel}

The properties of T91 stainless steel where estimated from generic stainless steel properties[10] as well as tensile stress strain curves on proton irradiated and pristine T91. A Young 's modulus of 200 $\mathrm{GPa}$, and a yield strength $0.5 \mathrm{GPa}$ was estimated from the tensile tests. The details of the yielding mechanisms were unclear. They were somewhat ductile, possibly involving some plastic deformation, but failure was ultimately by of fracture. The Poisson's ratio, $\nu=0.3$, and density, $\rho=8000 \mathrm{~kg} / \mathrm{m} 3$, were taken to be those of generic stainless steel. Brittle fracture was modeled by taking the yield strength equal to the failure strength, resulting in a failure strain, under uniaxial stress, of $0.25 \%$. This is a simplification of the material response, allowing the utility of the approach to be evaluated for genuinely brittle material. Separate plastic yield and limit (failure) surfaces are available in the general modeling approach. Fuel cladding is typically a cylinder of thickness $\approx 1.0 \mathrm{~mm}$. The grain size of T91 stainless steel is approximately $25 \mu \mathrm{m}$. This provides a natural scale for material heterogeneity although this approach models grain scale physics approximately.

\section{Tensile Specimen Simulations}

To experiment with this approach to modeling brittle failure, tensile tests to failure are simulated. First the homogeneous case is examined to assure that simulation parameters accurately replicate quasi-static loading. The tensile specimens are $4 \mathrm{~mm}$ long, with square cross-section, $1 \mathrm{~mm}$ x $1 \mathrm{~mm}$.

\section{Homogeneous Results}

First the case where all particle have the same failure parameters are investigated. Each material point fails when the maximum principal stress reaches $500 \mathrm{MPa}$. When a material point fails it no longer carries any tensile stress but still resists compression. For a uniaxial tensile test the maximum principal stress is the tensile stress. 


\section{Bulk Stress/Strain}

The force on the platens is measured during the simulation and divided by the current specimen platen contact area to generate a bulk specimen stress, as would be measured in a physical test. Bulk failure should occur at tensile stress $500 \mathrm{MPa}$ for these simulations regardless of numerical resolution. This is achieved as seen in Figure 1, where bulk stress-strain curves are depicted for various numerical resolutions. The horizontal line indicates the failure stress. Specimens fail via development of a planar surface of failed particles when this stress is reached and the specimen stress drops immediately. To assure that the rate of extension is quasi-static, faster and slower rates of extension were simulated. The results in Figure 2 depict extension rates as number of wave crossing times, i.e. the number of times an elastic wave propagates across the length of the specimen during the simulation. The previous results on numerical resolution are for 100 wave crossing times. Results for extension both twice as fast and twice as slow indicate that nearly quasi-static extension is achieved at 100 wave crossing times. For the faster extension case (50 wave crossing times), oscillations in bulk stress are evident due to wave transients. The slowest extension rate (200 wave crossing times) give the best results, but is also more computationally expensive. The intermediate rate gives reasonable results for modest computational effort and is used in all following simulations.

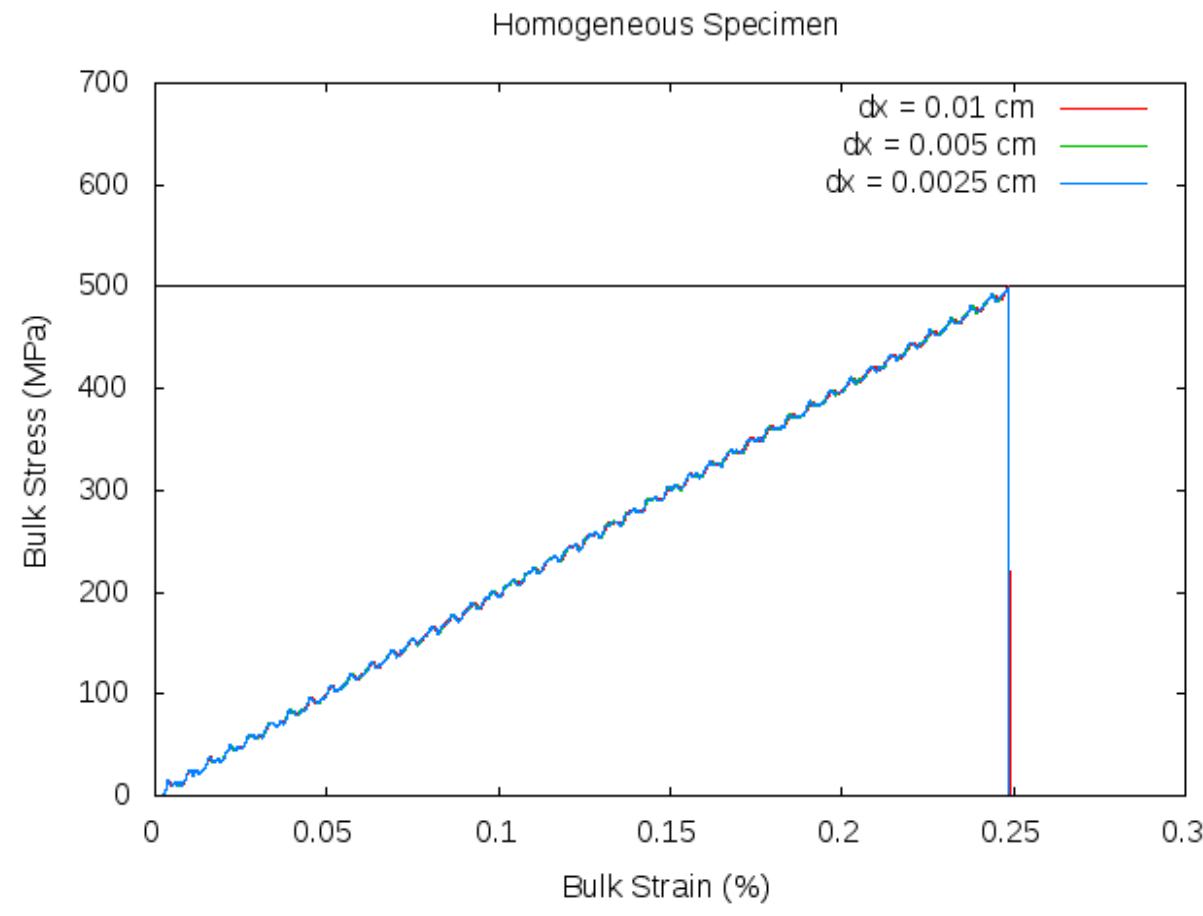

Figure 1 Bulk stress-strain curves for various numerical resolutions. 


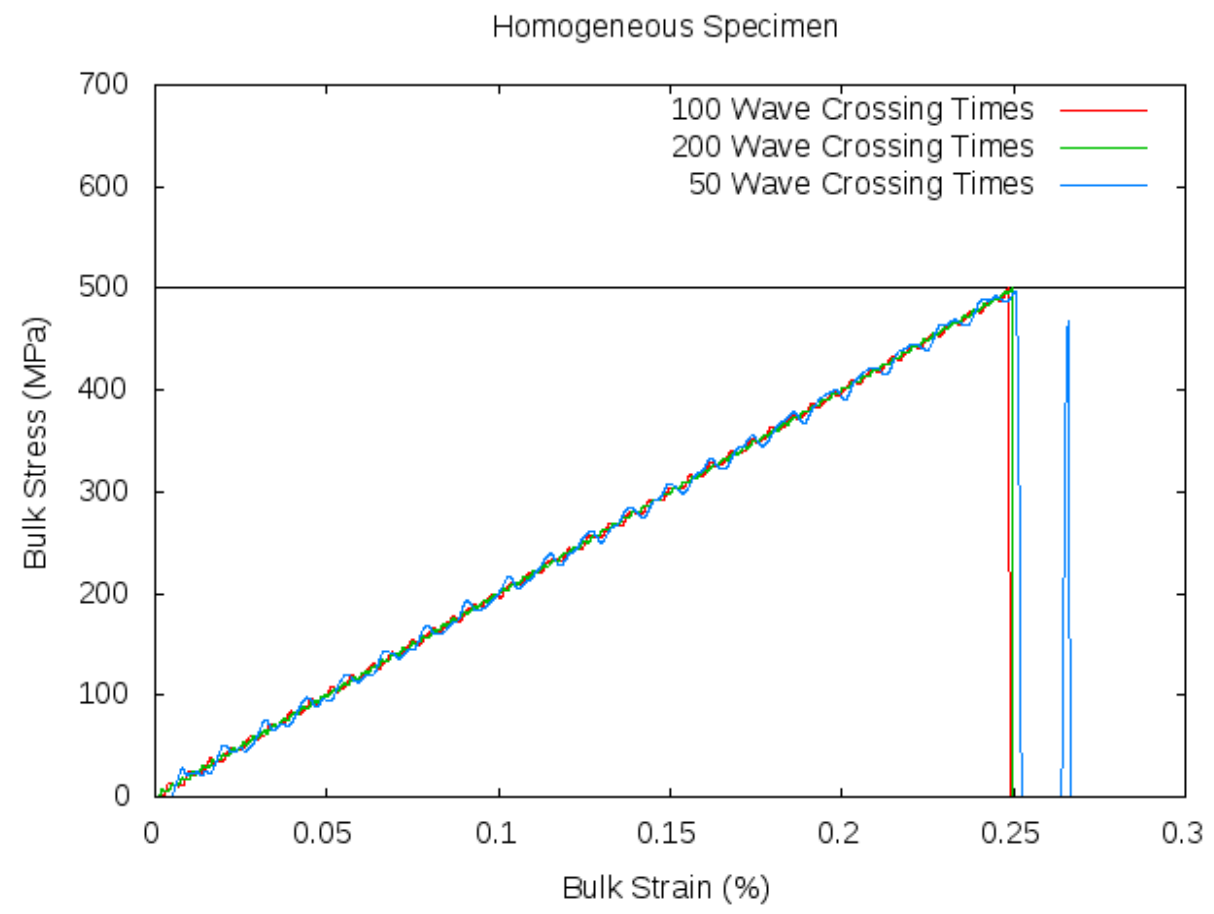

Figure 2 Bulk stress-strain curves for extension rates. 


\section{Failure Patterns}

The failure patterns developed soon after the maximum bulk stress is reached are depicted in Figure 3 for the various numerical resolutions used. Failed particles are colored red. In all cases a complete plane of failed particles develops perpendicular to the axis of extension. As may be seen from the bulk stress strain curves in Figure 1, all cases fail at the same bulk stress. Were the results truly quasi-static, all particles would fail simultaneously at the failure stress. Here the exact location of the failure plane is determined by stress wave transients and varies with numerical resolution. Once a complete failure plane develops the specimen is effectively fractured and the remaining intact material unloads.
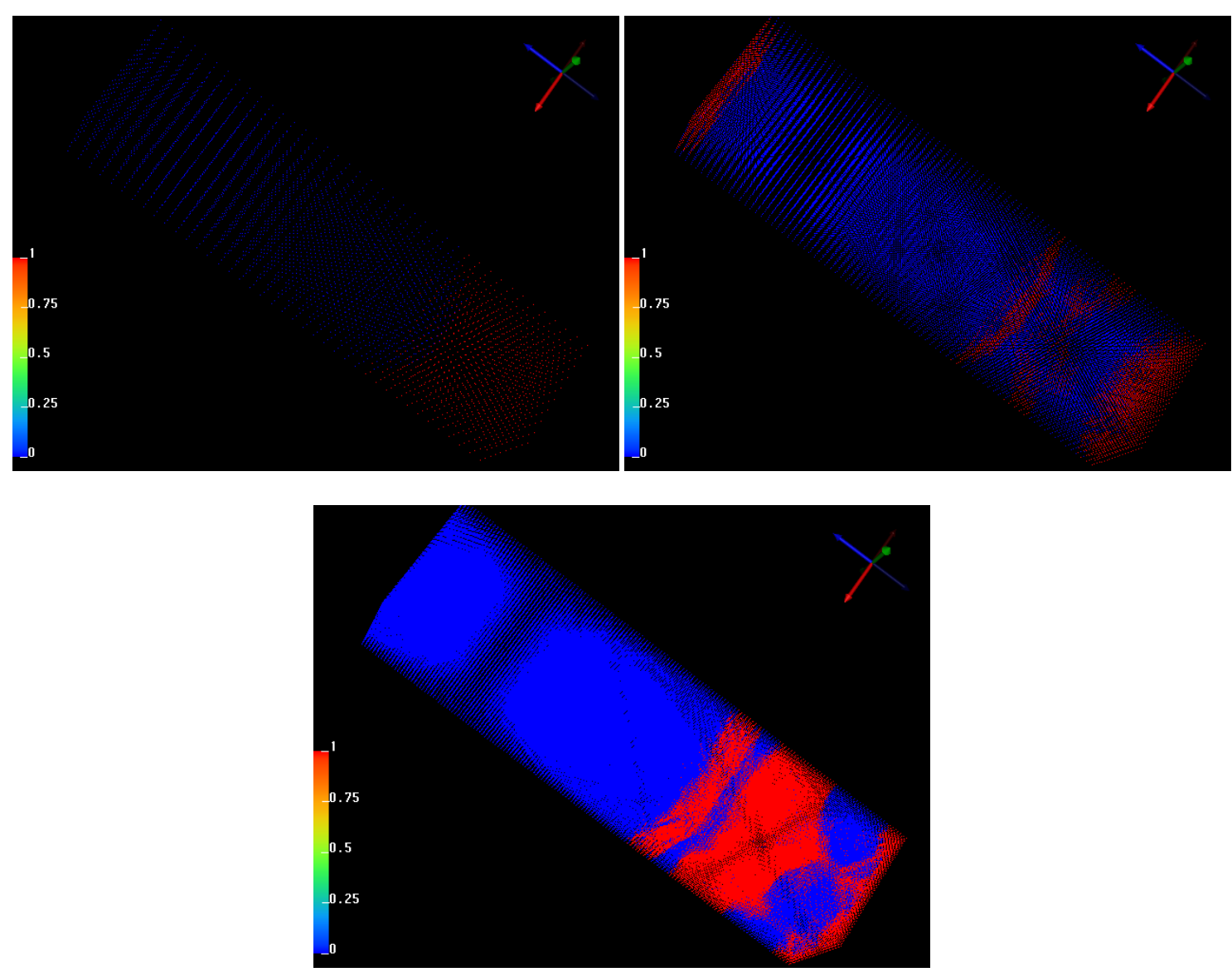

Figure 3 Failure patterns for the various numerical resolutions (cell size of $0.01 \mathrm{~cm}$, top left, $0.005 \mathrm{~cm}$ top right, and $0.0025 \mathrm{~cm}$ bottom) 


\section{Heterogeneous Results}

Next explicit material heterogeneity was introduced by varying the failure stress particle by particle in accordance with a Gaussian distribution. The mean of the distribution is $500 \mathrm{MPa}$, the same as for the homogeneous case. The standard deviation is $10 \%$ of the mean.

\section{Bulk Stress/Strain}

Bulk stress-strain curves for various numerical resolutions are depicted in Figure 4. As would be expected, heterogeneous specimens fail sooner than homogeneous ones on account of having weaker particles. It was conjectured that once the material heterogeneity distribution was sufficiently represented that the bulk failure stress would converge. This appears to be the case for the smallest cell sizes. Higher resolutions, or smaller cell sizes, provide a better sampling of the material heterogeneity distribution, as may be seen in Figure 5.

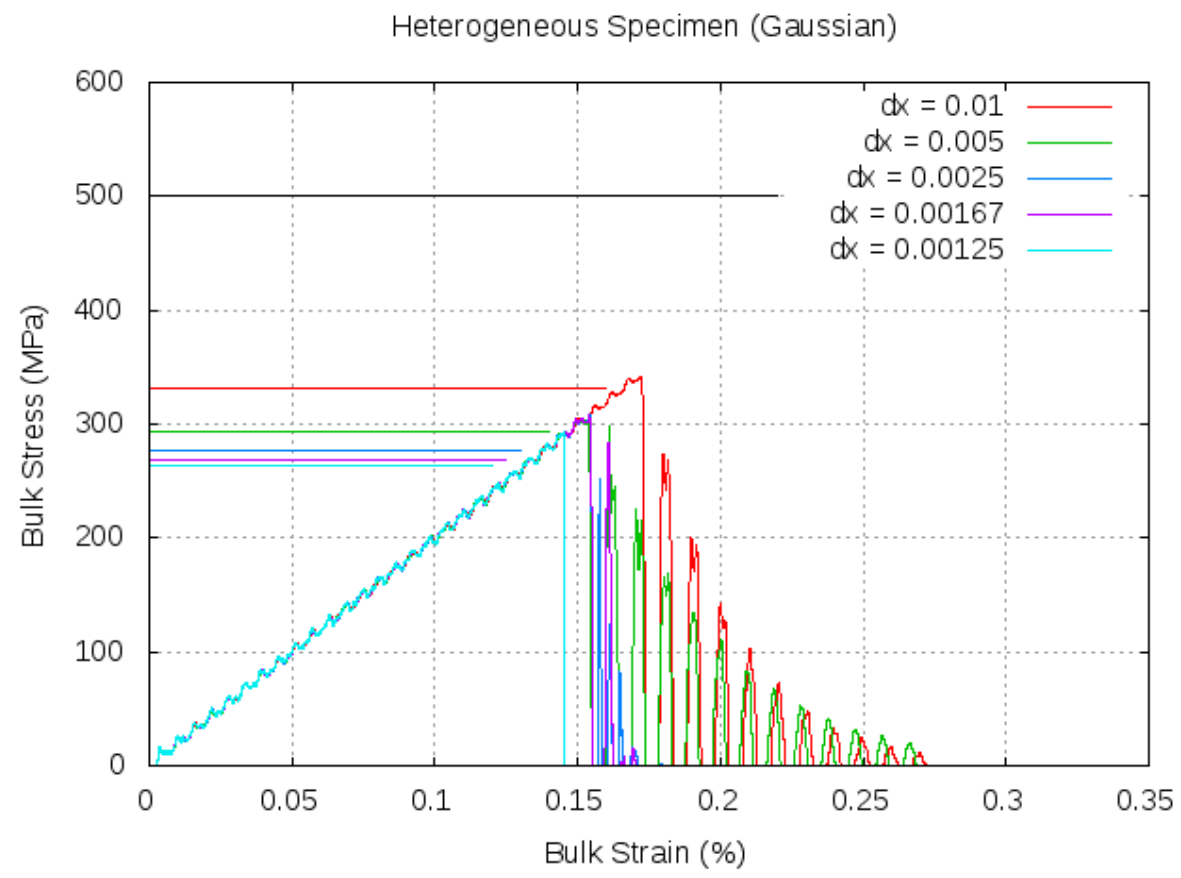

Figure 4 Bulk stress-strain curves for heterogeneous specimens at various numerical resolutions.

Colored horizontal lines in Figure 4 indicate the weakest particle failure stress, with the colors corresponding to numerical resolution. An alternate conjecture to convergence of bulk failure stress with numerical resolution is that the weakest particle dictates when failure occurs. This will be referred to as "weakest link" failure, and is also found to apply for certain cases as is explored below. 


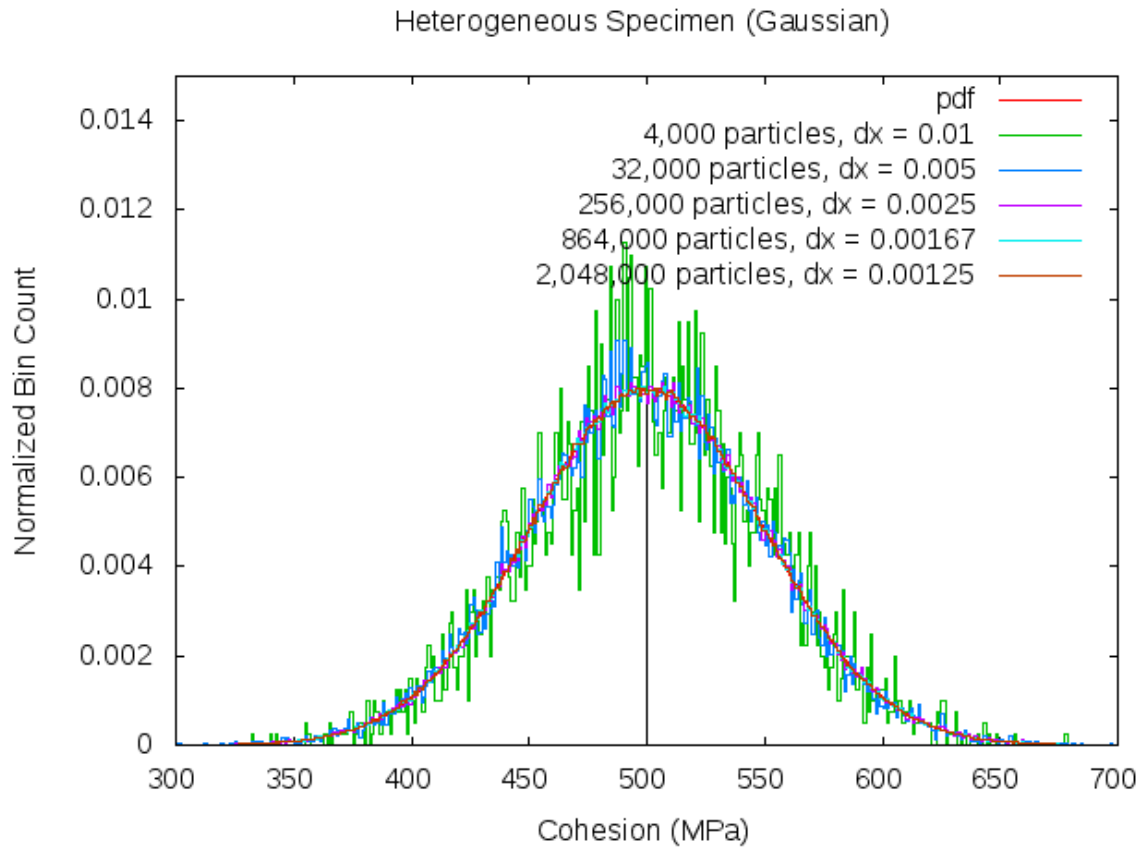

Figure 5 Material heterogeneity failure stress pdf, and histograms of particle failure stresses for various resolutions. The pdf is Gaussian with standard deviation $10 \%$ of the mean.

\section{Failure Patterns}

As for the homogeneous cases, it was found, in all cases, that a complete failure plane (all the way through the sample) was only found after the peak bulk stress. The failure patterns are depicted in Figure 6. The failure patterns are similar for all resolutions although the exact location depends on resolution. While wave transients may play a role, the various numerical resolutions also necessarily correspond to different realizations of material heterogeneity.
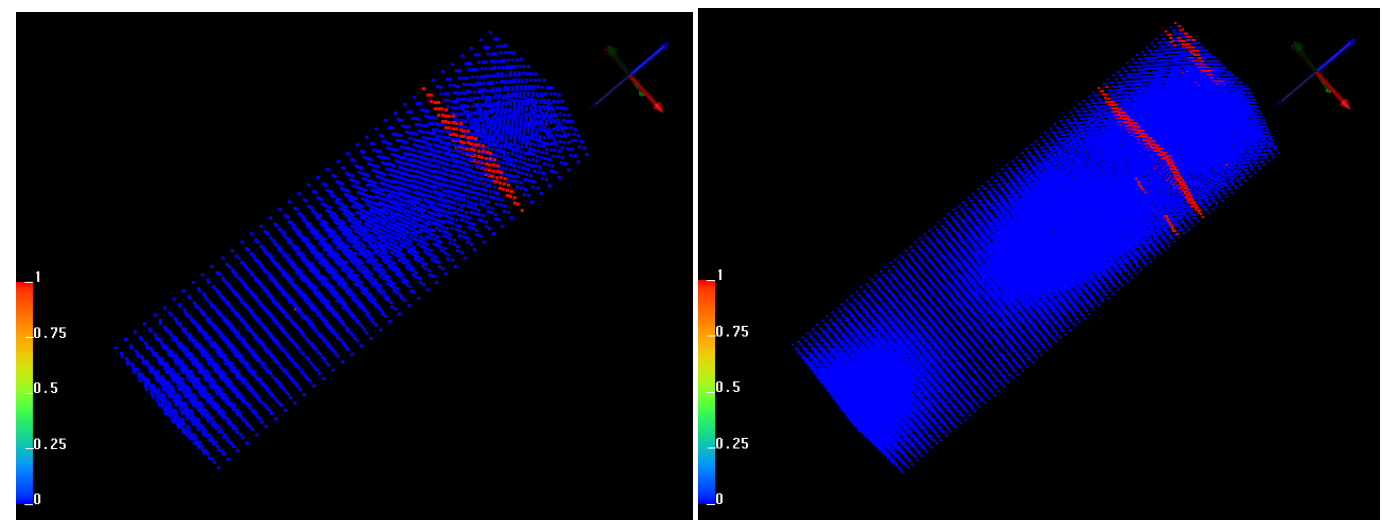

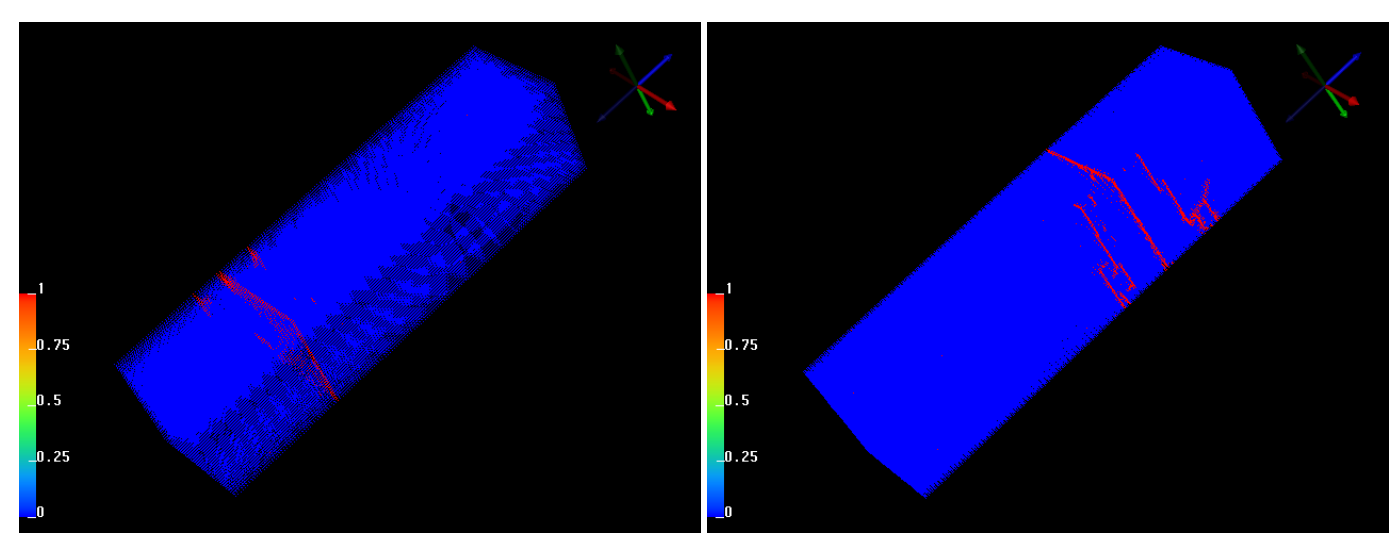

Figure 6 Failure patterns for various numerical resolutions (cell size of $0.01 \mathrm{~cm}$, top left, $0.005 \mathrm{~cm}$ top right, and $0.0025 \mathrm{~cm}$ bottom left and $0.00167 \mathrm{~cm}$ bottom right)

Since the material properties (besides failure stress) are uniform, the exact solution is one of constant stress and all numerical resolutions are capable of resolving this solution w/o error (the "error" there is, is on account of stress wave transients). The primary effect of numerical resolution is that it determines how many random samples of the failure stress distribution are taken, and hence how accurately the pdf is represented in the numerical simulation. A secondary effect, redistribution of stress post (individual) particle failure will take place amongst fewer particles at lower resolutions, may also play a role. The first particle that fails might be expected to trigger development of the bulk failure pattern under the weakest link hypothesis. However, the first particle to fail is separated temporally and spatially from the failure plane in all simulations where it could be located (full state output is only generated at finite intervals). For the cell size $=0.01$ $\mathrm{cm}$ case, the first particle to fail is the single red one on the left side. Consequently weakest link failure does not appear to occur for this case. Failure patterns are very similar at the different resolutions, but occur at different places.

\section{Heterogeneous Specimen RVE study}

To follow up on the previous results a new suite of runs was developed to assess the impact of "degree of heterogeneity", i.e. the influence of narrower and broader material heterogeneity distributions. A Gaussian distribution is again used, with the standard deviation taken to be various fractions of the mean (which remains at $500 \mathrm{MPa}$ ). Across $1 \mathrm{~mm}$ then, are 40 grains. A resolution of $0.0025 \mathrm{~cm}$ is a natural resolution to represent grain scale heterogeneity, and is used in all simulations. Here an representative volume element (RVE) study is performed. The particle size is identified with the grain size, $25 \mathrm{um}$. So the resolution remains constant, and larger and larger sample sizes simulated to find the RVE size. Based on previous results, it seemed we could afford to simulate quasistatic loading by allowing 200 wave crossing times (the time required for a wave to propagate the axial distance of the tensile specimen) over the course of the loading (tension to $0.25 \%$ strain). The end result is that the all specimens are pulled at the same velocity and larger 
specimens require proportionally longer simulation times to reach the same strain. Specimen sizes are identified by length 1 , which ranges from $0.01 \mathrm{~cm}$ to $0.1 \mathrm{~cm}$, corresponding to 256 to 256,000 particles, or grains. As explored previously, representation of the material heterogeneity is dependent on the number of grains, or the number of times the pdf is randomly sampled. In turn, a more broad pdf requires a higher sampling density than a narrower one (if the histogram bin size is kept constant). These trends can be seen in the histograms below for which the bin size is $1 \mathrm{MPa}$. It is unclear what bin size is most appropriate for these comparisons. However, this particular value results in the largest specimen sizes very accurately representing the pdf.
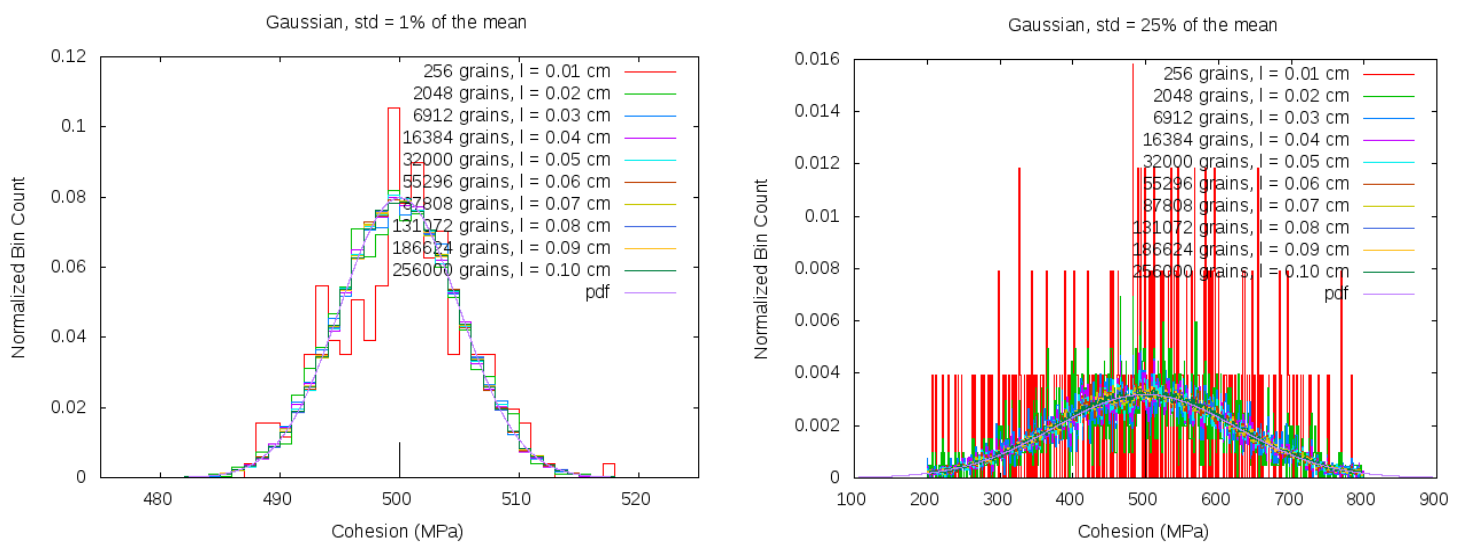

Figure 7 Material heterogeneity failure stress pdfs, and particle failure stress histograms for various resolutions. The standard deviation is $1 \%$ of the mean (left) and $25 \%$ of the mean (right).

\section{Bulk Stress/Strain}

Figure 8 shows bulk uniaxial stress strain curves for various degrees of material heterogeneity and various RVE sizes. Each RVE size is distinguished by a color. The black horizontal line indicates the failure stress of a homogeneous specimen (500 MPa). The colored horizontal lines indicate the minimum value of failure stress in a given RVE (a function of the sampling procedure and the number of samples, or "grains", or particles), i.e. first particle failure. As the material heterogeneity distribution is broadened, specimens of all sizes fail sooner, as would be expected. 

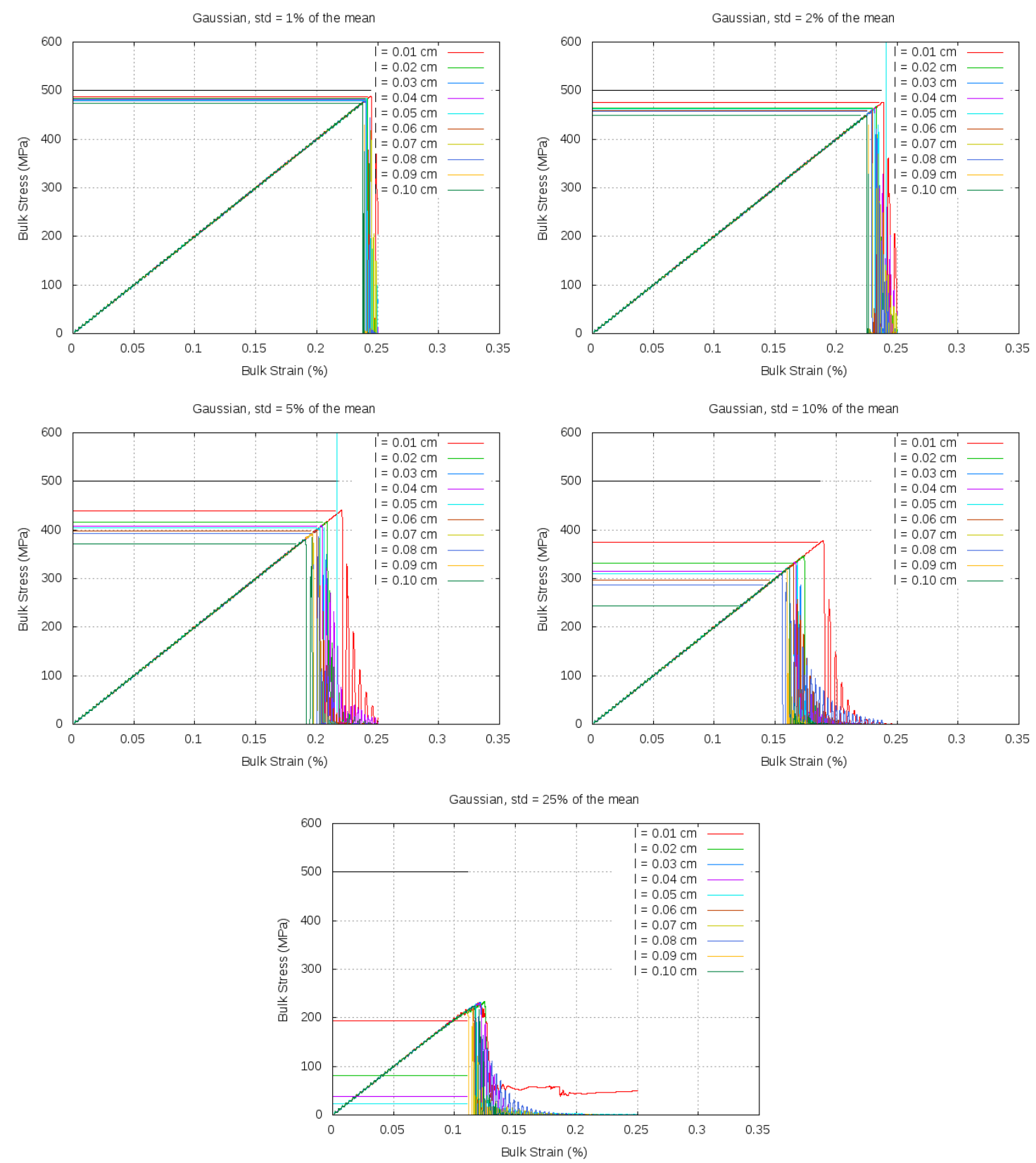

Figure 8 Bulk stress-strain curves for heterogeneous specimens for various degrees of material heterogeneity and various RVE sizes. 

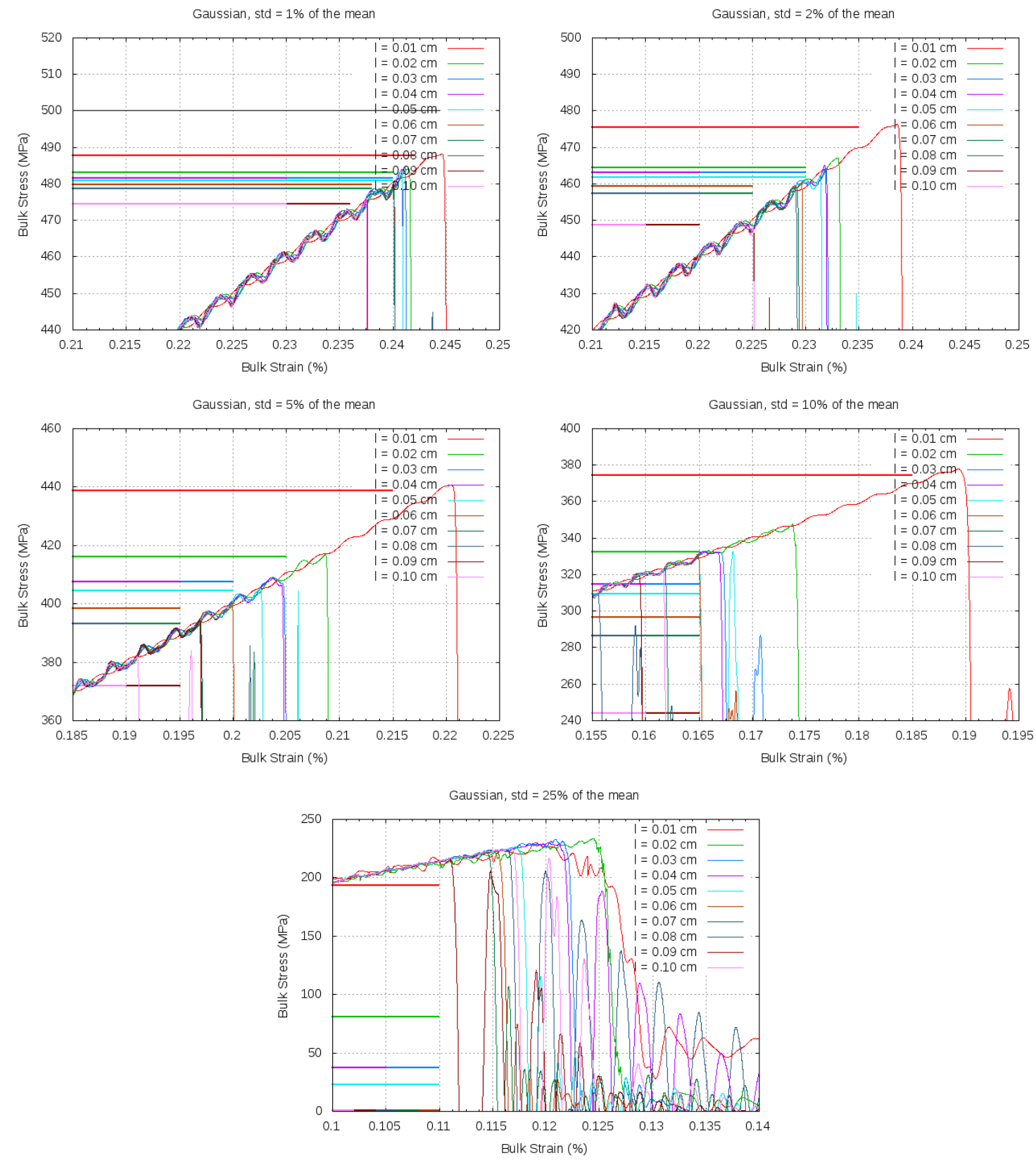

\section{Figure 9 Close-ups of bulk stress-strain curves for heterogeneous specimens for various degrees of material heterogeneity and various RVE sizes.}

Figure 9 depicts the same uniaxial tension stress strain curves, but zoomed in to distinguish failure points for the various RVEs. All strain axes cover a range of $0.4 \%$, so it can immediately be seen that the failure strains are tightly clustered for the narrowest material heterogeneity distribution and that the failure strains cover a broader range as the material heterogeneity distribution is broadened. 
This trend continues for material heterogeneity distributions with standard deviations of $1 \%-10 \%$ of the mean value. For the broadest material heterogeneity distribution, this trend is reversed, with failure strains again more tightly clustered. It appears that for the narrow material heterogeneity distributions (standard deviations from $1-5 \%$ of the mean) the bulk failure stress is closely correlated with the weakest particle failure stress. Several of the test specimens have the same value of minimum failure stress, in these cases the horizontal lines have different colored segments, indicating all cases with this minimum failure stress. As the distributions are broadened, the correlation between first particle failure and bulk failure disappears. For the broadest material heterogeneity distribution, failure strains are not even ordered with specimen size, occurring fairly randomly between 0.11 and $0.125 \%$ bulk strain. It should be noted that, for all simulations, one wave crossing time corresponds to a bulk strain increment of $0.00125 \%$, the spacing of the minor tick marks on the bulk strain axis. Because the bulk stress measurement is made at one end of the specimen, depending on where the failure surface develops, it may take as long as one wave crossing time, or $0.00125 \%$ bulk strain, to be detected. Thus the failure strain measurements may have as much as $0.00125 \%$ strain error, i.e. failure strains a minor tick mark apart are not distinguishable. For all cases, the same random number seed was used to generate a Gaussian distribution of grain failure stresses. Because of this, the various specimens are not completely independent. For a given degree of material heterogeneity, the next largest specimen size first assigns the exact same sequence of grain failure stresses, and then adds to it. Because the of the particle assignment pattern, relative locations are altered, and each specimen size can probably be considered independent. However, it results in the monotonicity of the minimum failure stress with specimen size. Each specimen size has the same minimum failure stress as the next smallest specimen unless one of the additional grains is assigned a lower value. This tends to be less frequent as the specimen size is increased. A second consequence of using the same random number seed is that specimens of a given size, but different degrees of material heterogeneity, are scaled versions of one another. The relative grain locations are identical, and each grain is assigned a failure stress that is proportional to the corresponding grain in a specimen with a different degree of material heterogeneity. The proportionality constant is the same for every grain. Hence specimens of the same size, but differing degrees of material heterogeneity, have proportional failure stresses, grain by grain. For the narrow material heterogeneity cases (standard deviation $1-$ $2 \%$ of the mean), bulk failure is correlated with first grain failure, i.e. the minimum failure stress. In the cases where it could be determined (most of them), the first particle failure always developed into the bulk failure. For broader material heterogeneity cases (standard deviation $10-25 \%$ of the mean) first particle failure is not correlated with bulk failure, which happens much later. This transition is especially noteworthy in view of the self similarity of same size specimens, with differing degrees of material heterogeneity (as described above). Bulk failure planes are found at different locations for self similar specimens with narrow and broad material heterogeneity distributions. Two self similar specimens $(1=0.1 \mathrm{~cm})$ with different failure planes are shown in Figure 10. On the left, the standard deviation is $1 \%$ of the mean, on the right it is $25 \%$ of the mean. On the left, the first particle to fail is in the failure plane. On the right, failure occurs throughout the specimen and eventually a failure plane develops. The intermediate material heterogeneity case 
is a crossover case. For the smaller specimen sizes failure is generally initiated at the weakest particle. For larger specimens the bulk failure plane generally develops elsewhere.

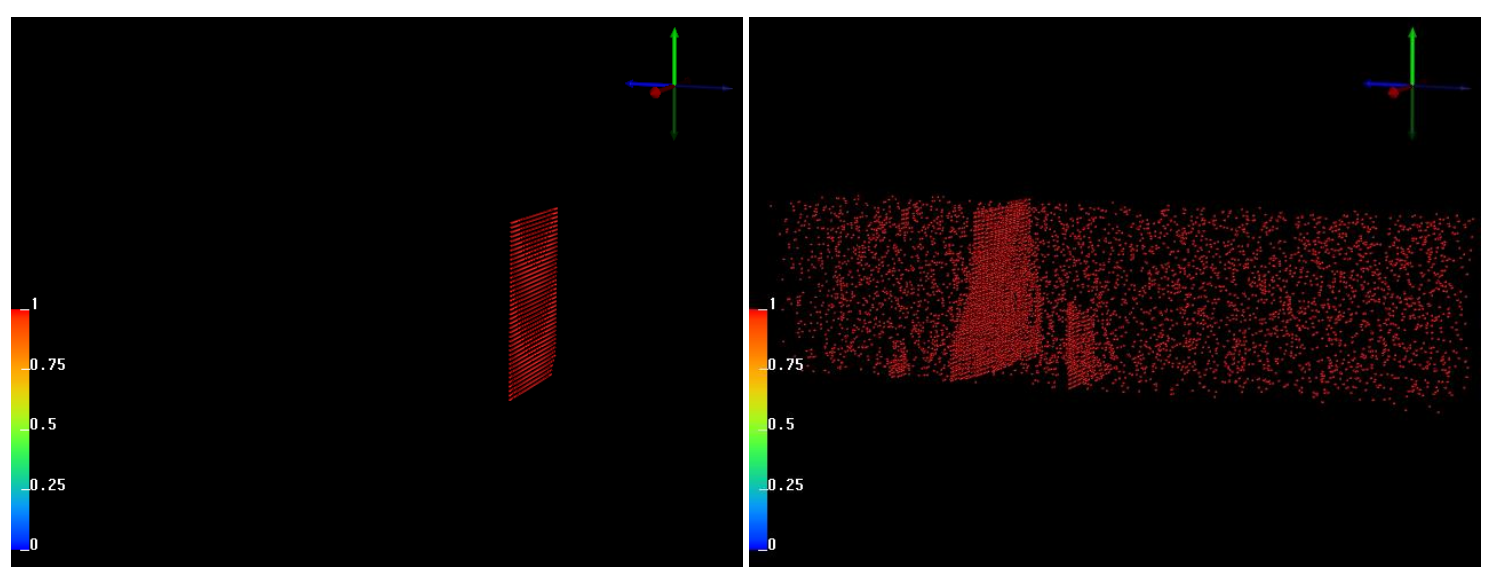

Figure 10 Self similar specimens with different failure planes. Only failed particles are shown.

Various random number generator seeds, in this case for the largest RVE size, give a feel for the variability associated with completely independent material heterogeneity realizations in Figure 11. Axes are scaled as in the previous close-ups. For narrow distributions the variability is directly related to variability in the weakest particle represented. For broad distributions, bulk failure variability is quite tightly clustered, and barely distinguishable based the boundary measurement for the broadest material heterogeneity case (bulk failure strains are all almost within one minor tick mark). 

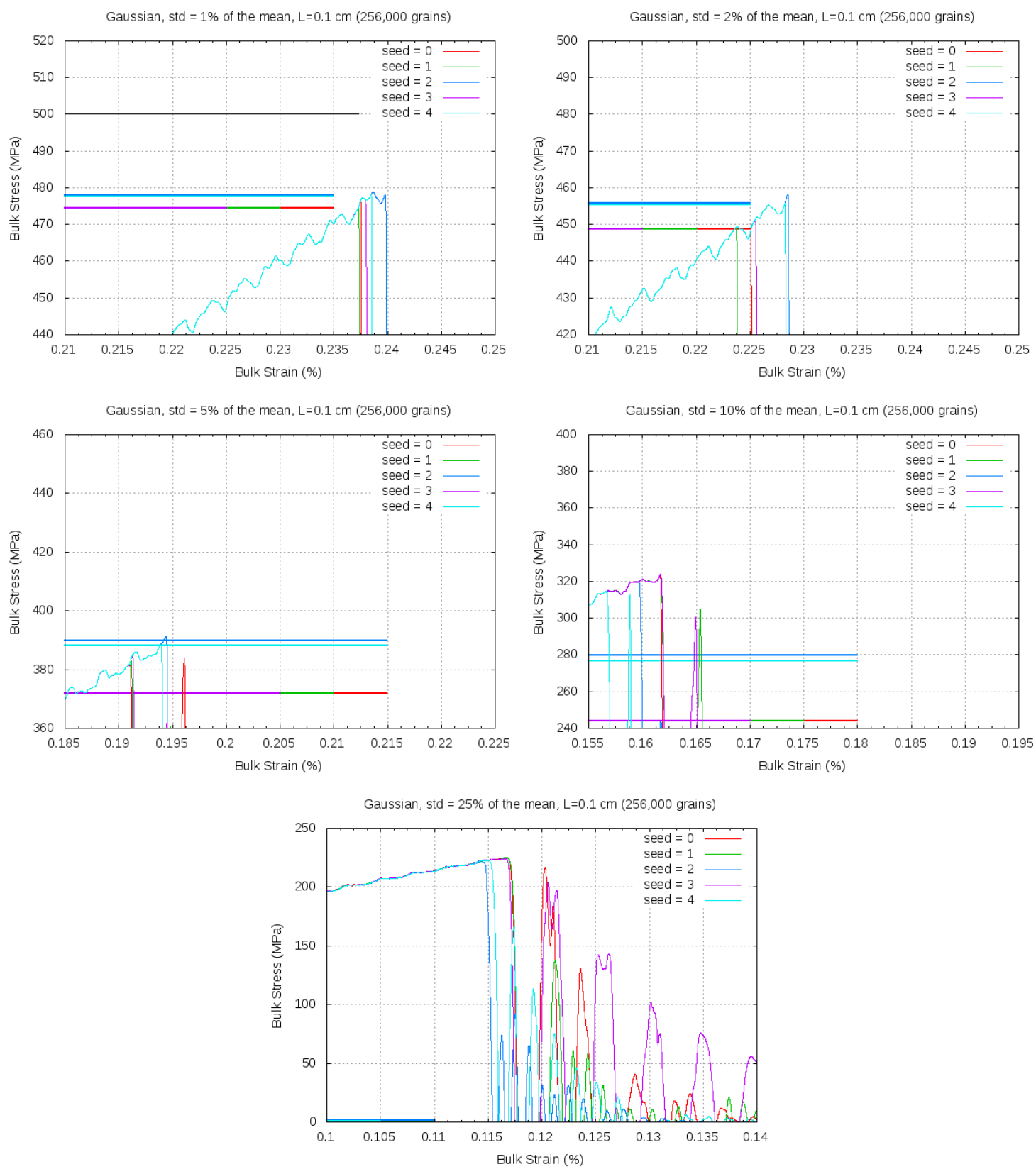

Figure 11 Close-ups of bulk stress-strain curves for heterogeneous specimens for various degrees of material heterogeneity and various random number seeds.

The intermediate case is especially interesting. As suggested by the bulk stress strain curves (and first particle failure lines), for seeds 2 and 4 the first particle failure develops into bulk failure. For the other seeds, the first failed particle may be seen away from the bulk failure planes. The failed particles may be seen in Figure 12 for this case. 


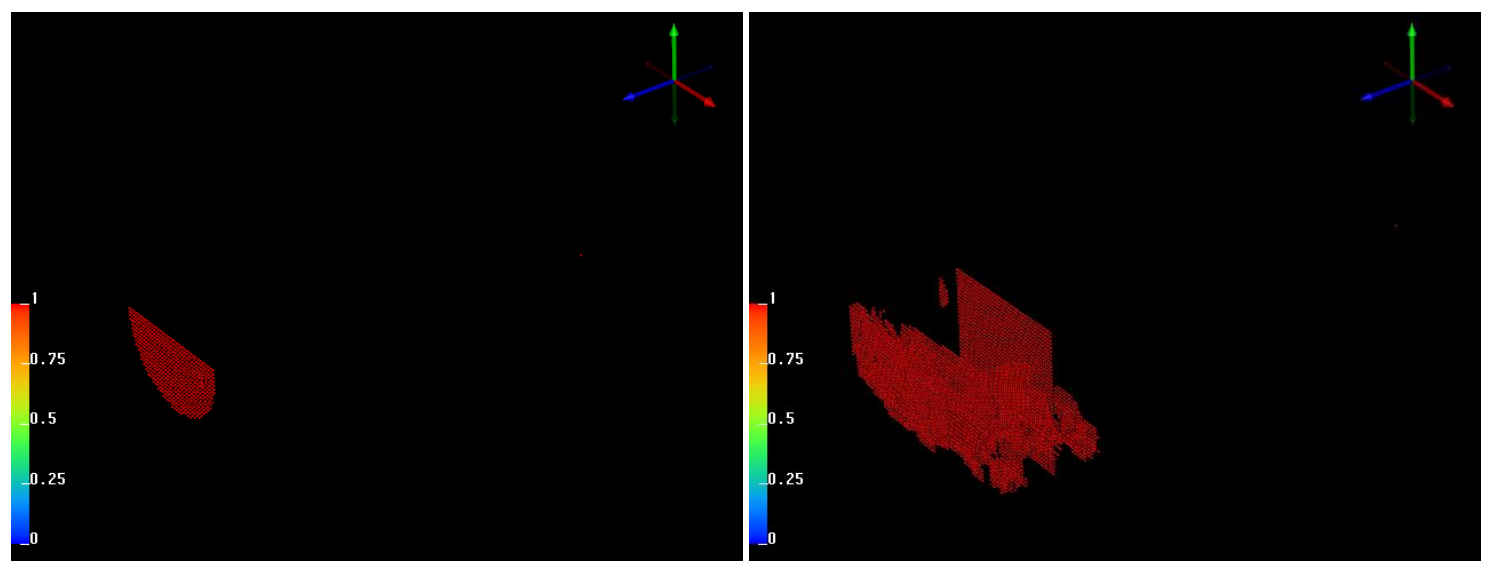

seed 0

seed 1

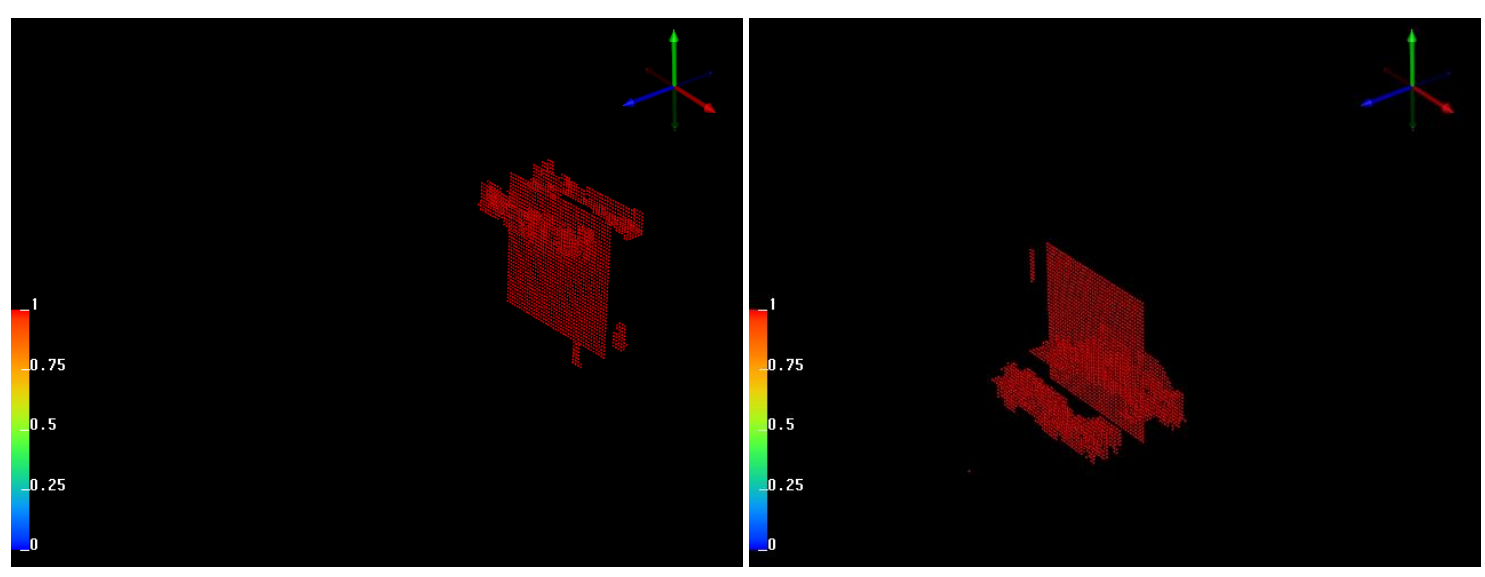

seed 2

seed 3

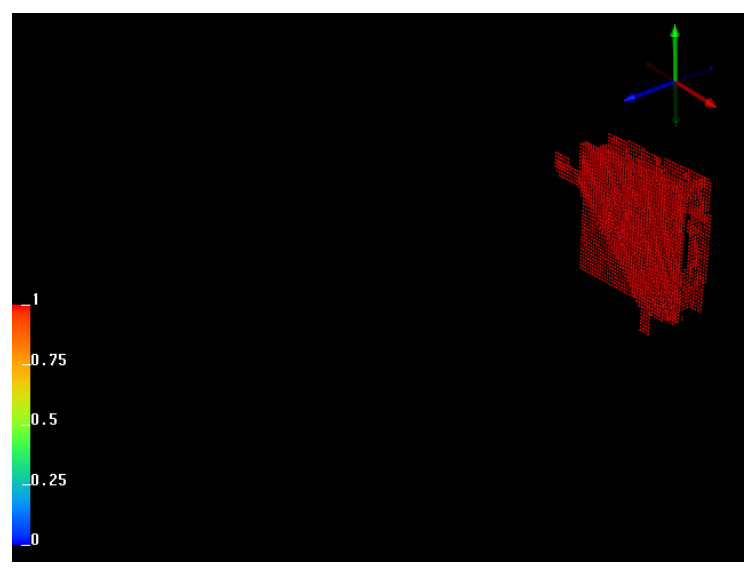

seed 4

Figure 12 Failure surfaces for various realizations of material heterogeneity (various random number seeds). 


\section{Preliminary Fuel Cladding Simulations}

As an approximation to the thermal mismatch loading experienced by nuclear fuel cladding, is loading by internal pressure. One quarter of a T91 fuel cladding cylinder is simulated. The inner diameter is $0.45 \mathrm{~cm}$ and the thickness is $1 \mathrm{~mm}$. Preliminary simulations, as depicted in Figure 13, suggest that failure plane spacing is independent of numerical resolution.
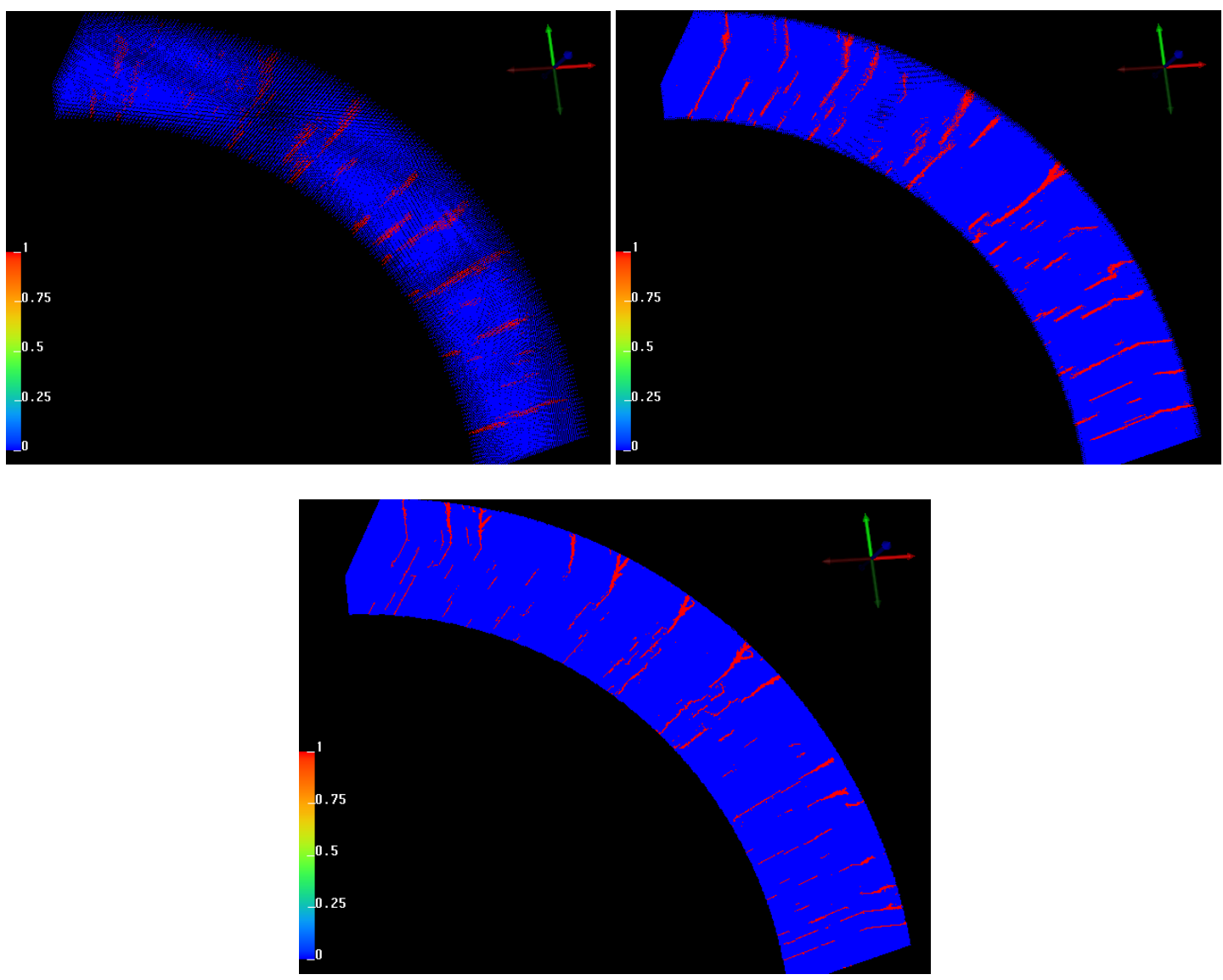

Figure 13 Preliminary simulations of pressurized nuclear fuel cladding. Failure patterns are qualitatively independent of numerical resolution. 


\subsection{Discretization Errors in the Material Point Method}

Numerical results to both establish RVE size and failure modes for various damage distributions, and establish appropriate constitutive behavior (including both damage and plasticity) for through-thickness variations in material response due to oxidation in service, were obtained using simulations of rectangular tensile specimens. When the geometry was changed to cylindrical, for simulating fuel cladding tubes, numerical artifacts were noticed. The artifacts are due to the resolution of circular geometry on a rectilinear grid. A mitigation strategy was employed, which greatly reduced the magnitude of the errors. However, material failure was found to be highly sensitive to small errors, even for heterogeneous materials. The result is that failure patterns are initiated by numerical errors. A possible solution is to use a newly developed variant of the Material Point Method (MPM), Convected Particle Domain Interpolation (CPDI).

\section{Discretization Errors in Pressurized Homogeneous Cylinder Simulations}

Fuel cladding is modeled as a circular cylinder with inner diameter $0.45 \mathrm{~cm}$ and thickness 1 $\mathrm{mm}$. These dimensions are typical of fuel cladding tubes. The tubes are spatially homogenous both in material properties and failure criteria. For the homogeneous case numerical solutions may be compared against analytical solutions for quasi-static deformation to assess solution quality. Results of initial scoping calculations are depicted in Figure 1, where the average hoop stress is compared with the static analytical solution for three different loading rates. Each loading rate is indicated by a number of wave crossing times (WCT) over which the internal pressure is ramped linearly to failure of the material based on a principal stress criterion. Only one quarter of the tubes are simulated, with symmetry boundary conditions applied at the computational boundaries.

WCT is calculated based on the cylinder thickness for these simulations. A value of 100 was initially selected based on the tensile specimen results, where it was found that ramping at that rate gave nearly quasi-static results. As can be seen from the top left inset in Figure 1, 100 wave crossing times is far too few. This is on account of the circumferencial equilibration required, i.e. the circumference is a much more relevant length scale for this problem. 
Average through-thickness hoop stresses are plotted for simulations at various loading rates. The smaller oscillations about the analytical solution, also plotted, indicate that 500 is a suitable number of wave crossing times to obtain quasi-static results.

The abscissa is time normalized by the final simulation time. For these simulations the final simulation time is selected to be the failure time, i.e. the time at which the failure criteria is reached assuming quasi-static deformation. For the simulations failure occurs much earlier than expected, at approximately 0.63 , rather than 1.0. This is on account of stress localization, on particles, where discretization errors are made. Failure patterns may also be seen in Figure 1 for the three cases. In all cases failure is initiated at the same locations, the symmetric angular locations of highest particle stress for this numerical discretization.
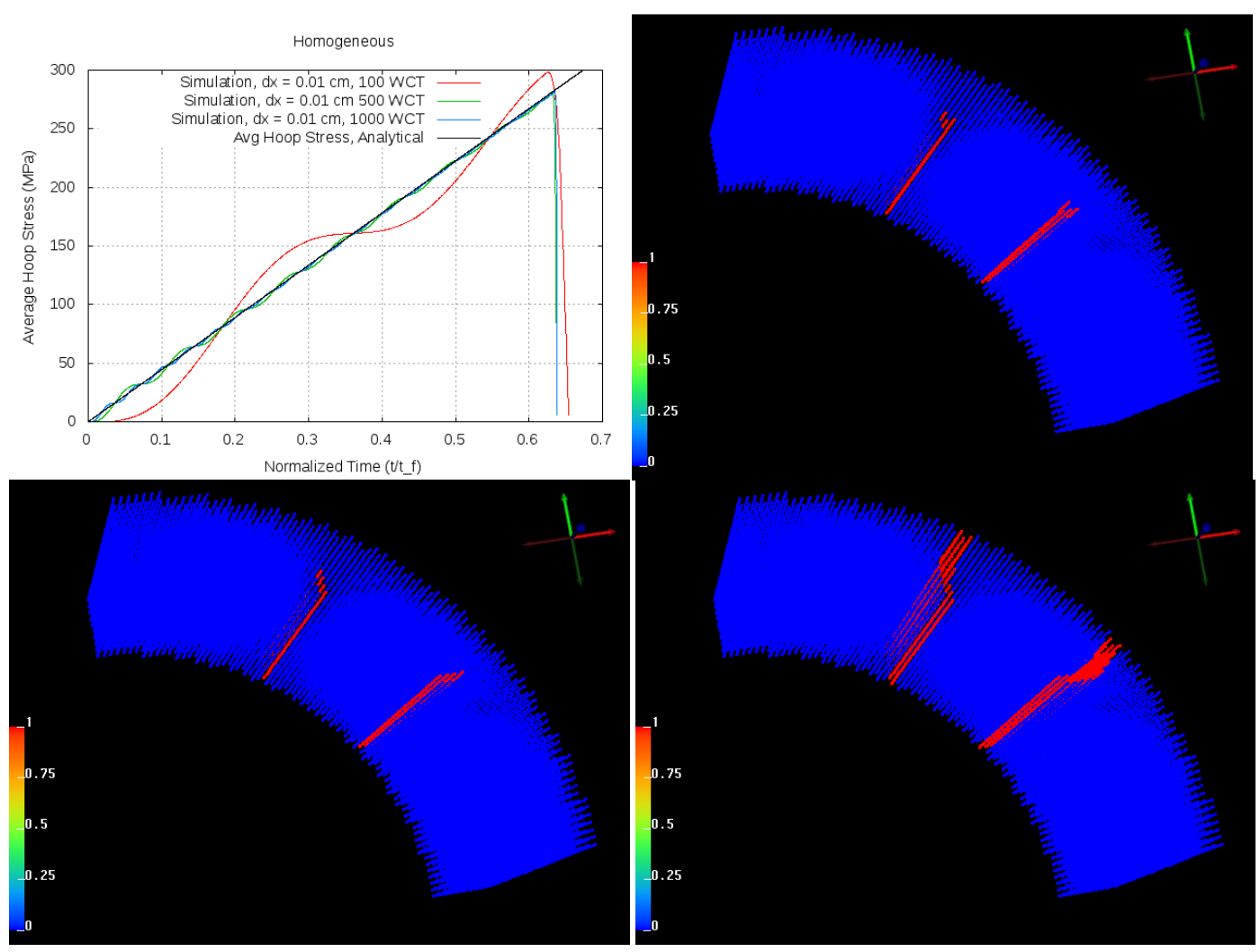

Figure 1 Homogeneous pipe pressurization at various rates.

When a circular object is resolved on a rectilinear grid, the surface is represented by stair-stepped particles. This has two consequences. First, the internal pressure, which is applied directly to particles, is applied non-uniformly. This is because the stair-stepped boundary presents different surface particle densities depending on angular location, and this is not accounted for in the pressure boundary condition algorithm. Second, the number of particles interpolating stress to a given computational node, where the governing equations are solved, varies at the interior and exterior surfaces. Since the stresses at the surfaces are independent of angular location, areas with fewer particles contributing to the stress at a node are required to carry higher stresses. Both of these effects generate stress concentrations near interior and exterior cylinder surfaces, and the stress concentrations determine the location of first failure. 
The effect is a function of numerical resolution, as illustrated in Figure 2 where pressurization at the intermediate rate is examined for three numerical resolutions. In the average hoop stress, normalized time inset, it may be seen that the effect is not monotonic. The middle resolution fails first, the lowest resolution second and the highest resolution last, spanning a range of $\sim 10 \%$. Different discretizations produce stress concentrations at different locations, as may be inferred from the failure patterns. Unfortunately, it's not a small effect. For all of these examples failure is occurring approximately $40 \%$ too soon due to numerical errors associated with discretization.
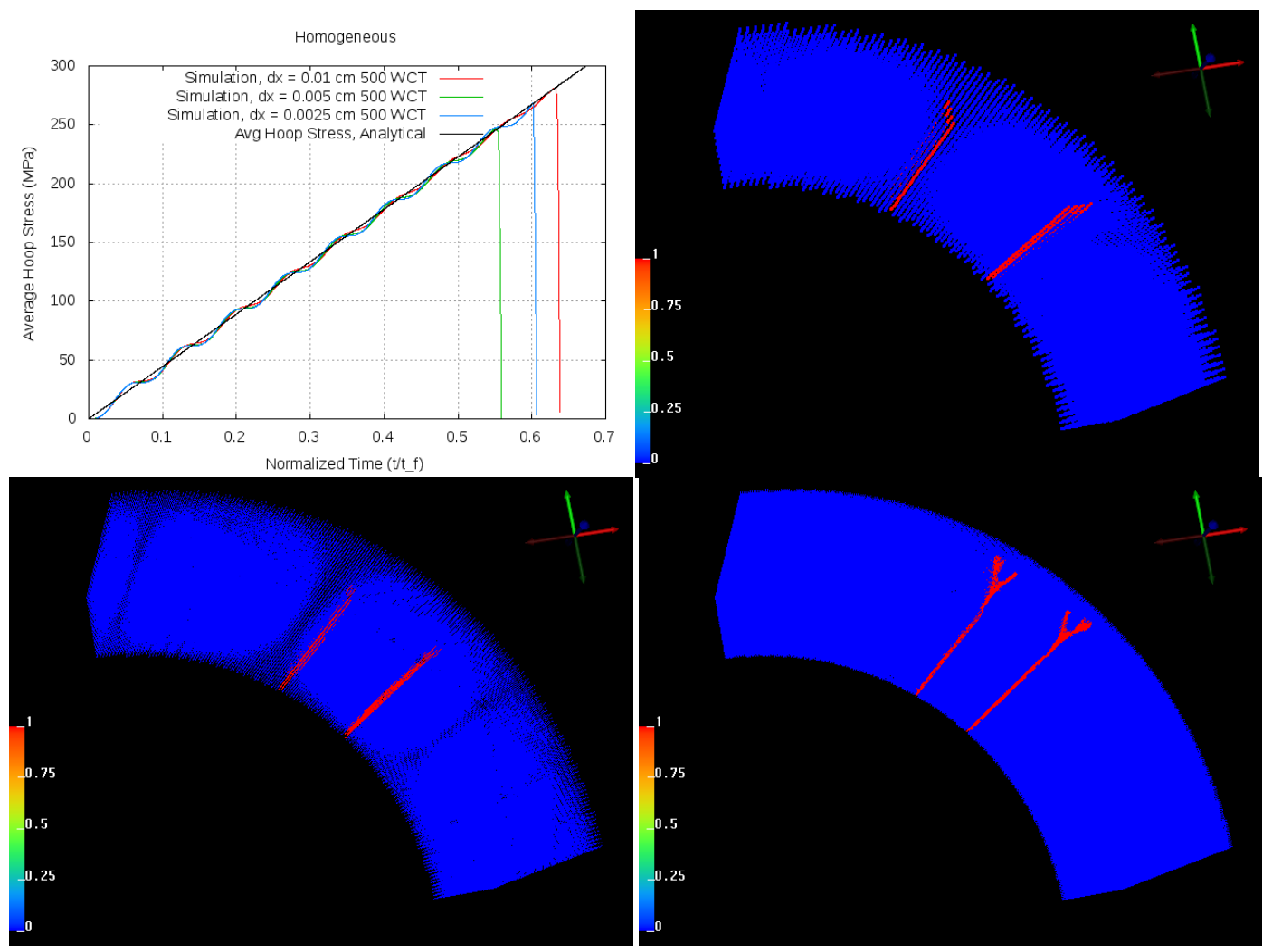

Figure 2 Homogeneous pipe pressurization at various resolutions.

\section{Reduction of Discretization Errors by Using a Liner}

Introducing a liner cylinder mitigates errors introduced at the internal surface stair-stepped boundary where the internal pressure is applied. The liner is chosen to perfectly elastic, with elastic constants matching those of the fuel cladding tube. This effectively removes the interior interface until the fuel cladding begins to fail. Pressure is applied to the liner, rather than directly to the fuel cladding. The same errors occur at the interior surface of the liner, but they are spatially removed the fuel cladding. The distance serves to reduce differences between the desired application of the interior pressure and that actually applied (with discretization errors), as experienced by the fuel cladding. It is an application of Saint-Venant's Principle.

Calculations were revisited using various thickness liners. Recall that the fuel cladding itself is 1 $\mathrm{mm}$ thick. Liners of thicknesses $0.5,1.0,1.5$ and $2.0 \mathrm{~mm}$ were tried. The cell size for these simulations was $0.05 \mathrm{~mm}$, so that there are nominally 20 cells across the fuel cladding thickness (rather than 10 for the first scoping study). The loading rate was changed slightly, to $1000 \mathrm{GPa} / \mathrm{s}$. This rate is much faster than used in burst test experiments, where rates on the order of $1 \mathrm{GPa} / \mathrm{s}$ 
are achieved. However, comparison to the static analytical solution indicates that errors due to stress wave transients are small. This is due to the very fast wave speeds in these structural steels. For T91, the elastic wave speed is $5.8 \mathrm{~mm} / \mu \mathrm{s}$.

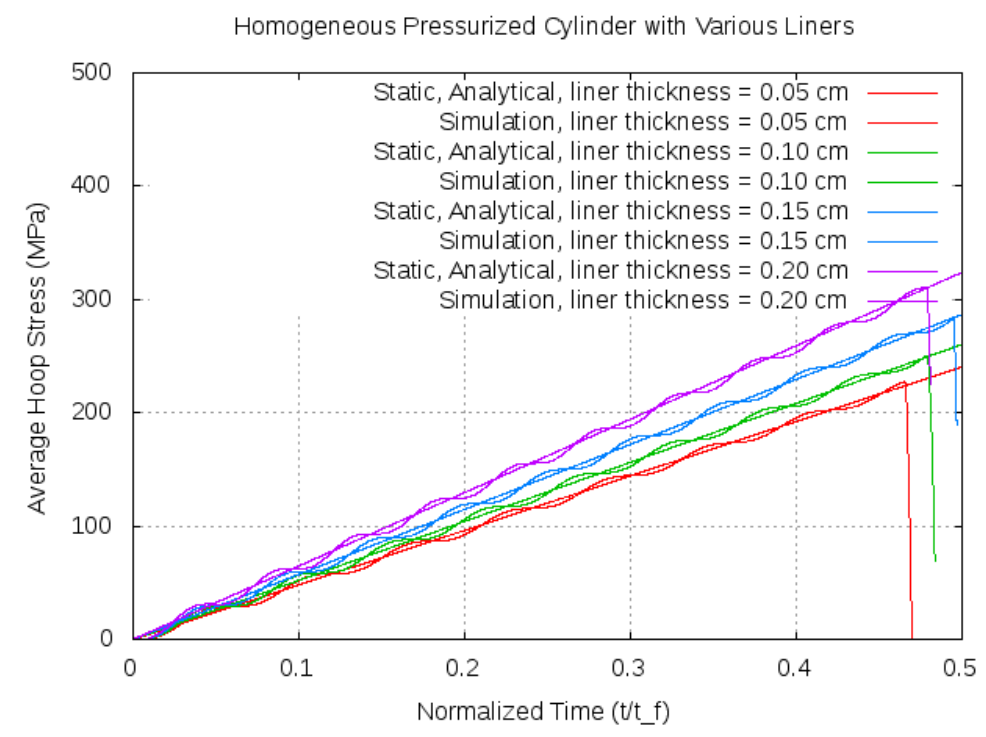

Figure 3 Homogenous pipe pressurization with liners of various thicknesses.

For the simulations in Figure 3 the failure time was selected to be half the simulation time, so that for quasi-static deformations, failure would occur at a normalized time of 0.5. As may be seen in Figure 3, failure occurs slightly earlier, as before. However, the errors are as greatly reduced. For the best case, a liner thickness equal to $1.5 \mathrm{~mm}$, the error is approximately $2 \%$. The error is sufficiently small that a significant fraction could be due to stress wave transients. Regardless, it was thought that the errors would be small relative to variations due to material heterogeneity.

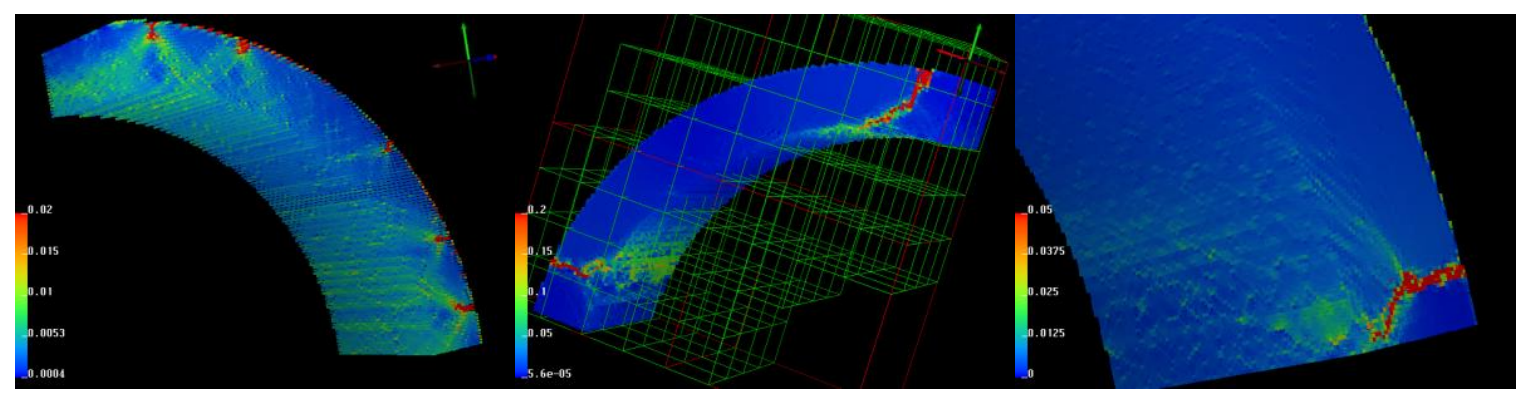

Figure 4 Failure patterns for various material heterogeneity realizations.

Unfortunately, the simulations suggest that errors are still driving the location of failure patterns, at least for the material heterogeneity parameters selected for modeling for through-thickness variations in material response due to oxidation in service. Various cases are depicted in Figure 4, each of which has slightly different material properties. The general symmetry of the failure patterns about the angular midpoint, as well as their persistent location, particularly near the left and bottom boundaries, suggest that failure is preferentially occurring at specific locations, most likely due to discretization errors. It is possible to greatly reduce these errors by tracking the deformation of the material point volumes, and using that information in the interpolation to the computational grid. The benefits of including particle volumes in the interpolation was recognized previously, where tracking of material point deformations in one-dimension was explored. Tracking material point deformations in three-dimensions is substantially more 
complicated, but clearly needed for accurate solutions of geometries that do not conform to a rectilinear grid. An expanding cylinder example is given and an initial version of CPDI algorithm has recently been implemented in Uintah for testing. The developers have been contacted to see if this version of the code may be obtained. 


\subsection{Implementation of Convected Particle Domain Interpolation (CPDI) for Error Reduction}

Numerical artifacts, due to the resolution of circular geometry on a rectilinear grid, were found to substantially degrade numerical solutions of pressurized fuel cladding pipes. Material failure was found to be highly sensitive to these errors, even for heterogeneous materials, which affected the resulting in failure patterns. As discussed in previous reports, various mitigation strategies were explored. A newly developed variant of the Material Point Method (MPM), called Convected Particle Domain Interpolation (CPDI), was found to substantially improve solution quality and was selected as the best way to address numerical artifacts. CPDI was implemented in Uintah for fuel cladding simulations.

\section{Implementation of CPDI in Uintah}

It is possible to greatly reduce simulation errors by tracking the deformation of material point volumes, and using that information in the interpolation of particle data to the computational grid. The benefits of including particle volumes in the interpolation was recognized previously, where an tracking of material point deformations in one-dimension was explored in the GIMP algorithm. Tracking material point deformations in three-dimensions is substantially more complicated, but clearly needed for accurate solutions of geometries that do not conform to a rectilinear grid. A production version of the CPDI algorithm has been implemented in Uintah. The implementation is limited, as it only applies to cylindrical geometries, but is sufficient for application to fuel cladding pipes. CPDI requires tracking particle sizes and locations relative to the underlying computational grid. The computational machinery to track this information and use CPDI interpolation was implemented in Uintah and tested for a single annulus as discussed previously. A production version of this capability required the development of a solid geometry object for an annular cylinder, which could then be combined with other solid geometry objects. For fuel cladding tubes, this allows concentric annuli to be discretized and assigned material properties consistent with radiation induced damage of inner and outer layers, as discussed previously. 
A body-fit particle discretization was implemented in the "smoothcylinder" geometry object in Uintah Computational Framework. An annular cylinder is divided into a specified number of annuli in the axial direction, and each annuli is discretized via concentric rings of particles. Two discretization schemes were implemented. In the first case the angular particle spacing was taken to be constant for each ring of particles. For the second case, the angular spacing was selected based on the spacing between concentric rings. The first scheme is the simplest, but has the drawback that particle sizes are smaller in each concentric ring closer to the cylinder axis. The second scheme generated approximately constant particle sizes throughout the cylinder.

Both discretization schemes are illustrated in Figure 1, for typical fuel cladding dimensions (1 mm thick tubes of inner radius $4.5 \mathrm{~mm}$ ) Particles are colored by volume (in cubic microns). Note that the color bar scales are substantially different in the two illustrations. For constant angular particle spacing the particle volumes vary between 14,100 and $17,200 \mu \mathrm{m}^{3}$, while for the other scheme particle volumes vary between 15,600 and $15,700 \mu \mathrm{m}^{3}$. The nearly constant particle volume scheme is of interest for modeling damage and failure where each particle is taken to approximately represent one grain in stainless steel tubes (with grain size $\sim 16,625 \mu \mathrm{m}^{3}$ ).

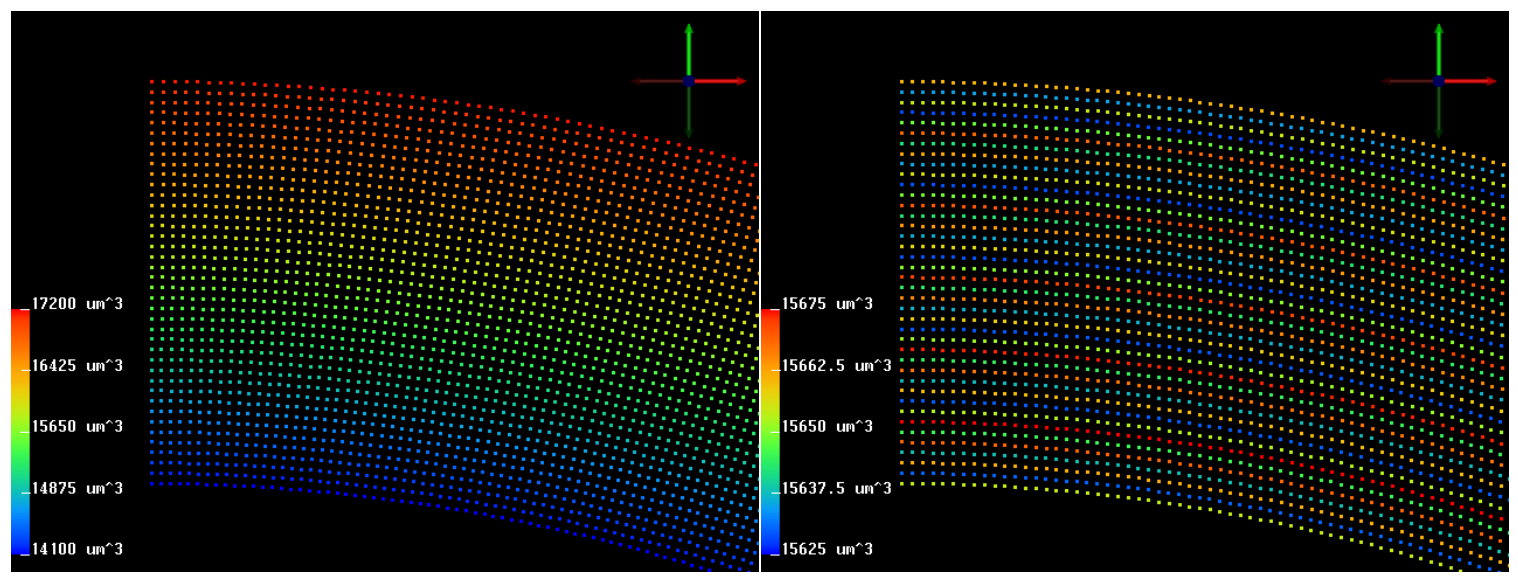

Figure 1 CPDI discretization schemes. Constant angular particle spacing (left) and approximately constant particle volumes (right). Particles are colored by volumes.

\section{Burst Test Simulations with Explicit Material Heterogeneity}

Preliminary results for burst test simulations, similar to those described in previous reports, are presented in Figure 2. These simulations used the nearly constant particle volume discretization scheme, and each particle was prescribed a failure stress consistent with a Gaussian distribution. Specifically, material properties are the "outer layer", brittle material properties previously presented (standard deviation equal to $25 \%$ of the mean failure stress). Three independent realizations of material heterogeneity were simulated.

Hoop stress is presented as a function of time in the top left frame of Figure 2, and provides an assessment of the dependence of failure on the details of material heterogeneity. The three different realizations of material heterogeneity simulated are indicated by the random number seeds used in the particle strength assignment scheme (in the random number generator). Maximum values of hoop stress vary by approximately $20 \%$ for the three different cases. Failure patterns are also depicted, and are distinct for the three cases, in contrast to previous simulations, where failure patterns where consistently found to have features associated with numerical artifacts. The new 
Uintah geometry will be used to revisit previous simulations of fuel cladding tubes with distinct, radiation induced damage in the inner and outer layers of the tubing.
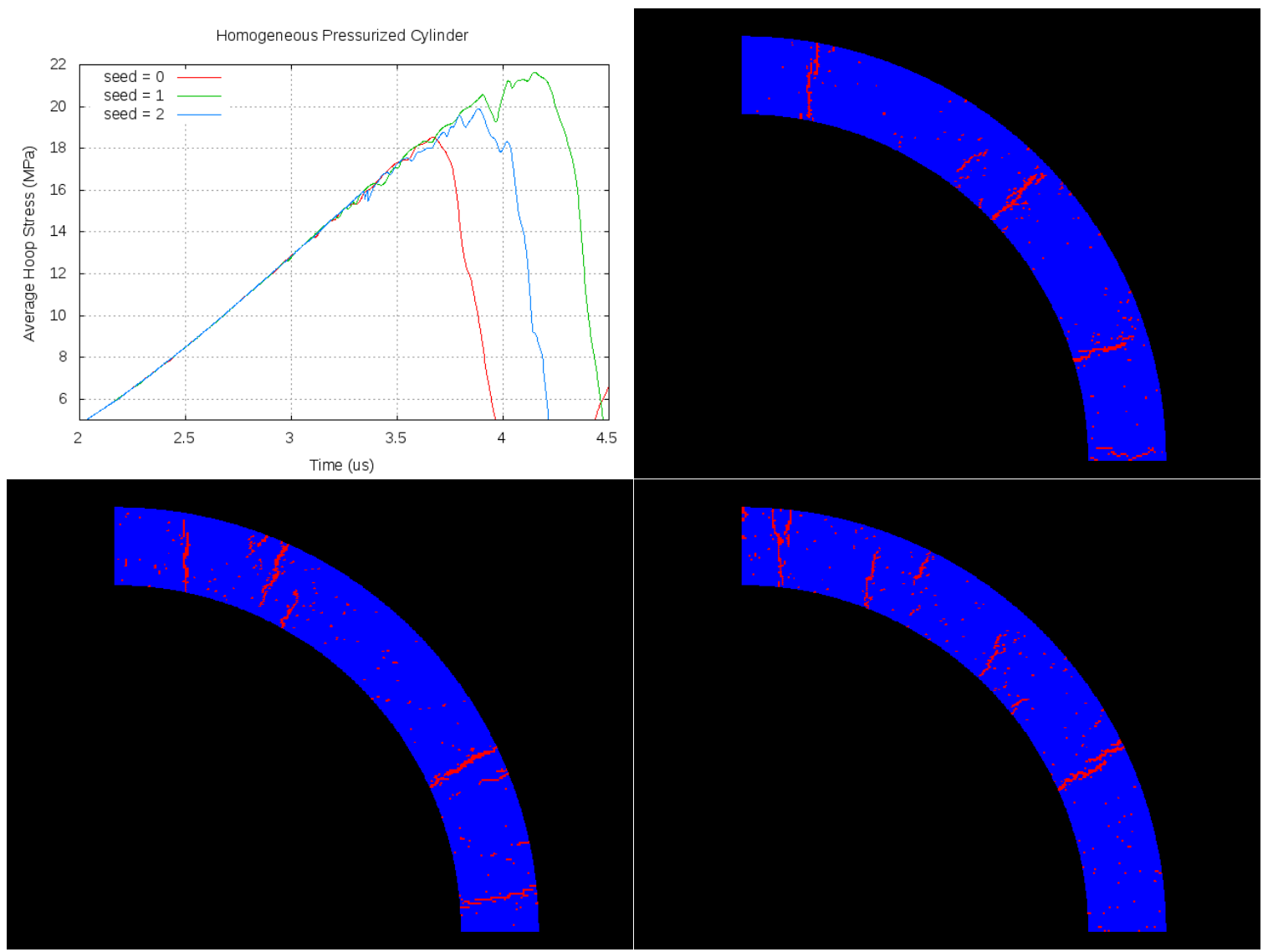

Figure 2 Results for simulations of pressurized fuel cladding with three independent material heterogeneity realizations. Bulk response (top left) and failure patterns (top right, seed $=0$, bottom left, seed $=1$, and bottom right seed $=2$ ). 


\section{Simulation of Fracture in Irradiated Iron using Molecular Dynamics}

\subsection{Introduction}

Steels continue to the be the material of choice for reactor core designs including the next generation Advanced Burner Reactors (ABR). In order to improve the safety and operating costs of these reactors, it is important to understand the short and long-term effects of irradiation on metals. Experimental studies that test the performance of reactor steel tubings and pressure vessels are absolutely necessary but expensive and long-drawn. The outcomes of such studies provide information on changes in mechanical properties due to irradiation exposure, however, the changes in micro-structural level is rather difficult to observe and understand. Simulation techniques such as molecular dynamics (MD) and Monte Carlo method can complement experimental studies by providing details on dislocation movement and micro-structural evolution.

Continuous exposure to neutron irradiation is known to be detrimental to the service life of metallic components. Due to combination of high pressures and temperature gradients along with neutron bombardment in reactors, various modes of failure have been reported, such as stress corrosion cracking, embrittlement, cavitation, and fuel-cladding interaction. High energy neutron bombardment on metallic surfaces lead to cascades of atomic displacements. These atoms are called primary knock-on atoms (PKA). PKAs experience a spike in kinetic energy which in turn lead to collisions with neighboring atoms in evolution of defects. Direct observations of the neutron induced damages in metals have been previously reported. Defect clusters and hardening are observed in iron exposed to neutron irradiation. Embrittlement also causes reduction in yield strength and fracture toughness of materials.

Molecular Dynamics (MD) is a popularly used tool to study irradiation effects on materials. High energy neutrons (up to $100 \mathrm{keV}$ ) collide with iron atoms which gain kinetic energy in this process. As energy of the cascade increases, the number of atoms in the system also increases. In this study, we focus on the interactions of propagating crack with irradiation induced displacement cascades using 
MD. Crack propagation along primary slip planes of perfect single crystal bcc-iron are simulated at different irradiation energies. We are not aware of previous numerical studies, focusing on the mechanics of crack propagation under the influence of irradiation.

\subsection{Computational Methodology}

MD simulations were performed using the Large-scale Atomic/Molecular Massively Parallel Simulator (LAMMPS) installed at High Performance Computing center at Oklahoma State University. Simulations were performed on a single crystal bcc-iron specimen comprising of 121,200 atoms. Figure 1 shows the generalized schematic of the specimen. An initial edge crack of 10a length was generated on the left edge of the specimen along (001) plane and [110] direction by turning off the interactions between the atoms located in the upper and lower surfaces of the crack. The specimen was oriented such that the crack propagation was investigated along [100]. Remote tensile strain was applied on the rigid group of atoms (boundary atoms) in the upper and lower boundaries of the specimen. The loading was displacement controlled with a constant displacement rate of $v_{y}=0.005 \AA / \mathrm{ps}$, in the $y$-direction. The groups of atoms in the upper and lower boundaries were kept rigid by setting the interaction forces to zero. Temperature of mobile atoms were initialized to $300 \mathrm{~K}$ using Gaussian distribution. During the simulations, thermostating was performed by velocity rescaling in the microcanonical ensemble (NVE), following the work by Campana et al. (2008). Atomic interactions were governed by many body $\mathrm{Fe}-\mathrm{Cu}-\mathrm{Ni}$ embedded atom method (EAM) potentials, developed by Bonny et al. (2009) for reactor pressure vessel steels. Periodic boundary condition along the thickness of the simulated model was implemented.

Irradiation was introduced in a square region of four lattice units in the center of the sample along the path of crack tip. The small size of irradiated region was necessary to prevent any spurious effects from sudden increase in energy due to irradiation. In order to simulate the interaction of pre-existing crack with irradiation damaged zone, sufficient time was allowed for the crack tip stresses to rise. At $330 \mathrm{ps,}$, irradiation effect was introduced in the form of kinetic energy (specifically temperature) 
Table 1: Relationship between energy of bombarding neutrons versus temperature of primary knock-on atoms (PKA) in bcc iron.

\begin{tabular}{ccc}
\hline $\mathrm{E}_{\text {neutrons }}(\mathrm{keV})$ & $\mathrm{E}_{\text {IronPKA }}(\mathrm{eV})$ & Eq. temp. $(\mathrm{K})$ \\
\hline 0.0145 & 1 & 11605 \\
0.145 & 10 & 116050 \\
1.45 & 100 & 1160500 \\
14.5 & 1000 & 11605000 \\
\hline
\end{tabular}

on all atoms in the irradiated region. This increase in kinetic energy is analogous to the collision of incoming neutrons on iron atoms. Four different irradiation levels of 1, 10, 100, and $1000 \mathrm{keV}$ were simulated and the results were compared. The kinetic energy was imparted to the individual atoms in terms of temperature assuming the relationship, $1 \mathrm{eV}=11,605 \mathrm{~K}$. The transfer of energy from the incoming neutrons to the iron atoms is considered as an elastic collision. The maximum energy transferred to the atom by the neutron is given by the equation (1) as per Turner (2007). The maximum ratio of energy $Q_{\max } / E_{n}$ is 0.069 for a neutron colliding with iron atom.

$$
Q_{\max }=\frac{4 m M E_{n}}{(M+m)^{2}}
$$

\subsection{Results}

In the absence of irradiation, crack propagation was found to be catastrophic after 300 ps. This corresponded to tensile strains of $1.2 \%$ which might be higher than those observed in experiments, due to the absence of macroscale plasticity. Similar observations in strains have been reported by others researchers. Prior to crack propagation, slip bands were emitted from crack tip towards the remote boundaries. Emission of slip bands typically requires lesser energy compared to crack tip nucleation. Figure 2 shows the snapshots of crack tip progression with varying irradiation energy levels. At zero and low energy levels $(\leq 1 \mathrm{eV})$, crack propagation was found to be brittle-like with smooth fracture surfaces. With increase in irradiation energy, the crack tip behavior was found be ductile-like. It is to be noted that there was no weak or preferred plane for crack propagation that was defined a priori in these simulations. The nature of crack propagation, straight or branched, was defined by the atomic layout and the crack tip parameters. 


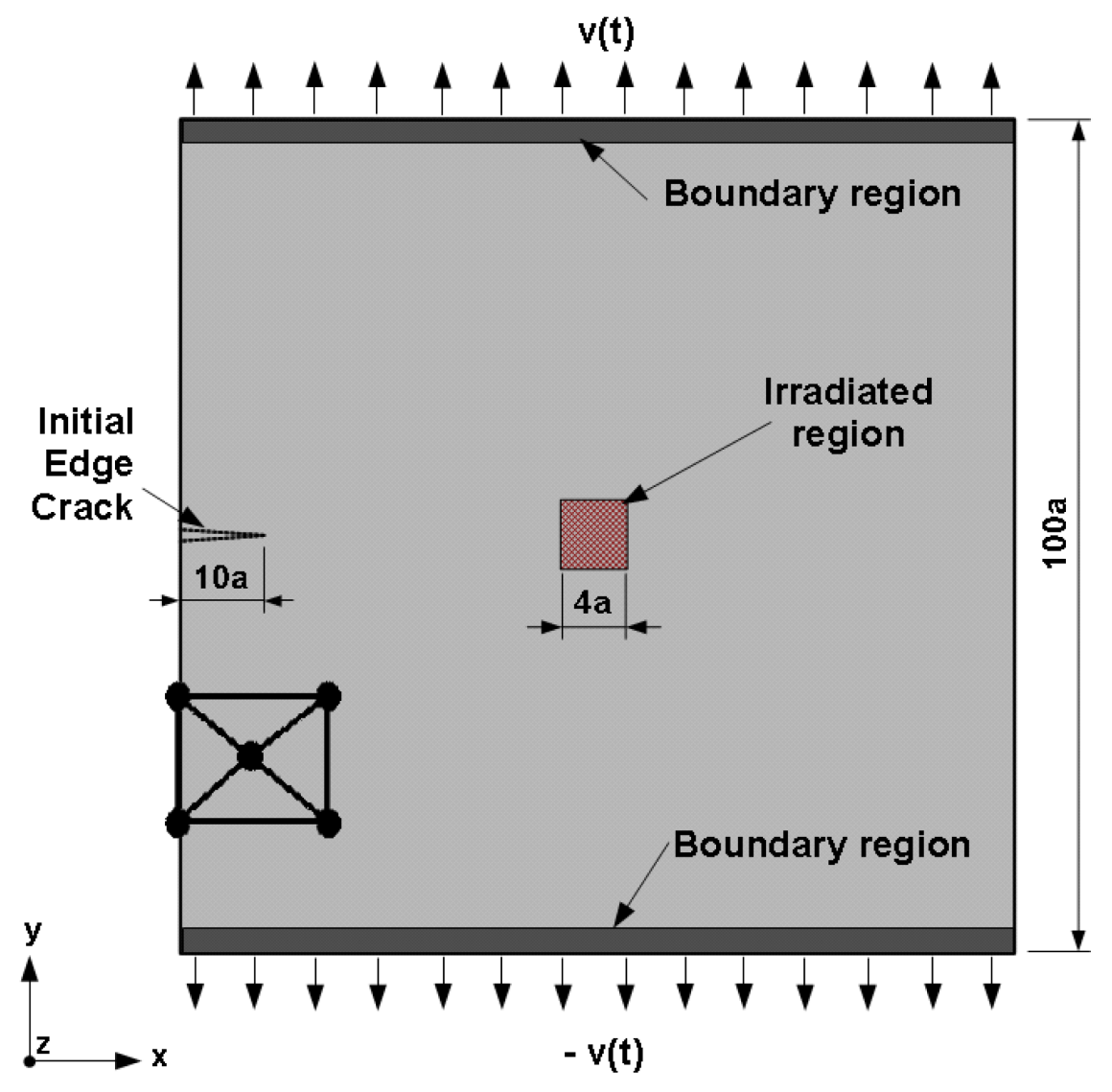

Figure 1: Generalized schematic of simulation of crack progression under irradiation 


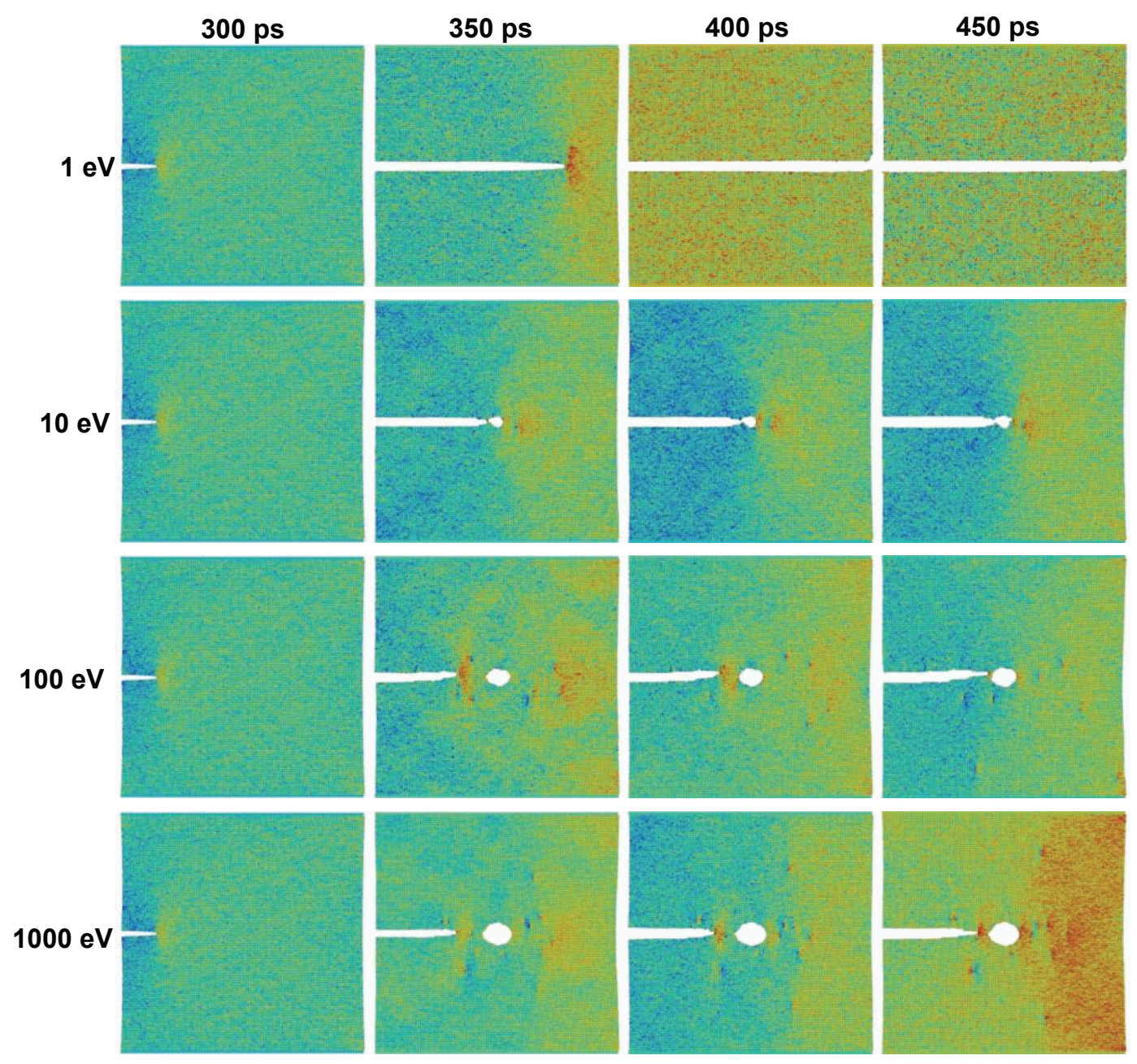

Figure 2: Snapshots of stress distribution $\left(\sigma_{y y}\right)$ during crack propagation at different irradiation (1 $\mathrm{eV}$ to $1 \mathrm{keV}$ ). After initial build up of crack tip stresses, irradiation is turned on at $330 \mathrm{ps.}$

Table 2 lists the values of crack progression, average velocity, and void size for different irradiation energies. Irradiation energies, in $\mathrm{eV}$, used in this study cannot be directly compared to that of nuclear irradiation. This is due to the simplification of the neutron-PKA interaction. For low energies of $\leq 1 \mathrm{eV}$, the crack traveled through the material, cleaving it into two halves. Although average crack tip velocities were around $90 \mathrm{~m} / \mathrm{s}$, instantaneous velocities were much higher, $500 \mathrm{~m} / \mathrm{s}$.

At higher irradiation energies, the crack propagation was arrested. High energy neutron 
Table 2: Crack lengths and crack tip velocities for different irradiation energies.

\begin{tabular}{cccc}
\hline \hline $\begin{array}{c}\text { Irradiation } \\
\text { Energy } \\
(\mathrm{eV})\end{array}$ & $\begin{array}{c}\text { Max. Crack } \\
\text { Length } \\
(\AA)\end{array}$ & $\begin{array}{c}\text { Avg. Crack } \\
\text { Tip Velocity } \\
(\mathrm{m} / \mathrm{s})\end{array}$ & $\begin{array}{c}\text { Irradiation } \\
\text { Void Size } \\
(\AA)\end{array}$ \\
\hline 1 & - & 95 & 0 \\
10 & 214 & 46 & 26 \\
100 & 154 & 31 & 42 \\
1000 & 146 & 29 & 55 \\
\hline
\end{tabular}

irradiations are known to cause local disturbances in the metallic lattice. However, in the presence of crack tip stresses irradiation affected zone experienced an outward push of atoms thereby creating a void in the material. Unlike typically observed line or point defects, the void is few ten Angstorms wide. This blunted the progression of crack tip. From table 2, we find that the void size increased with increase in irradiation energy. This 'annealing effect' of irradiation ahead of crack tip blunted the progression of crack. Moreover, the crack tip did not coalesce with the void in irradiation zone.

Simulations were also performed to study the effect of irradiation on an initial edge crack. Irradiation is initiated at time $t=10 \mathrm{ps}$. After $490 \mathrm{ps}$, the tensile strain on the sample was terminated but irradiation was continued. The irradiation energy varied from $1 \mathrm{eV}$ to $5 \mathrm{keV}$. Above $10 \mathrm{eV}$, the crack tip propagation was not found to be significant. The average velocity of the crack tip was also found to be very low, on the order of $1 \mathrm{~m} / \mathrm{s}$. No void generation was observed in the irradiation damaged region even at the higher energies. This leads to the conclusion that the irradiation produced an overall stress relaxation in the specimen. This relaxation prevented the accumulation of stresses in the crack tip from the remote loading. At very low values of irradiation, at $1 \mathrm{eV}$, the crack growth rate was found to be similar to the Case of crack propagation in an irradiation-free specimen.

\subsection{Conclusions}

Reactor steels undergo loss in service life with prolonged exposure to neutron irradiations. We used MD simulations to study the effect of irradiation energy on a pre-existing edge crack in bcc-iron single crystal. Irradiation induced damage in the material was more pronounced ahead of the crack 
tip leading to development of void in irradiated zone. The presence of void ahead of the crack tip inhibited the propagation of the edge crack. These nano-voids in the material inhibit failure resulting from growth of pre-existing cracks. Further investigation of pre-existing crack growth in irradiated polycrystalline materials might provide better understanding of the role irradiation in failure of metals. 